\title{
Relaxed Extragradient Methods with Regularization for General System of Variational Inequalities with Constraints of Split Feasibility and Fixed Point Problems
}

\author{
L. C. Ceng, ${ }^{1}$ A. Petruşel, ${ }^{2}$ and J. C. Yao ${ }^{3,4}$ \\ ${ }^{1}$ Department of Mathematics, Shanghai Normal University, and Scientific Computing Key Laboratory of Shanghai Universities, \\ Shanghai 200234, China \\ ${ }^{2}$ Department of Applied Mathematics, Babeș-Bolyai University, 400084 Cluj-Napoca, Romania \\ ${ }^{3}$ Center for Fundamental Science, Kaohsiung Medical University, Kaohsiung 807, Taiwan \\ ${ }^{4}$ Department of Applied Mathematics, National Sun Yat-Sen University, Kaohsiung 804, Taiwan
}

Correspondence should be addressed to J. C. Yao; yaojc@kmu.edu.tw

Received 1 September 2012; Accepted 15 October 2012

Academic Editor: Julian López-Gómez

Copyright (C) 2013 L. C. Ceng et al. This is an open access article distributed under the Creative Commons Attribution License, which permits unrestricted use, distribution, and reproduction in any medium, provided the original work is properly cited.

We suggest and analyze relaxed extragradient iterative algorithms with regularization for finding a common element of the solution set of a general system of variational inequalities, the solution set of a split feasibility problem, and the fixed point set of a strictly pseudocontractive mapping defined on a real Hilbert space. Here the relaxed extragradient methods with regularization are based on the well-known successive approximation method, extragradient method, viscosity approximation method, regularization method, and so on. Strong convergence of the proposed algorithms under some mild conditions is established. Our results represent the supplementation, improvement, extension, and development of the corresponding results in the very recent literature.

\section{Introduction}

Let $\mathscr{H}$ be a real Hilbert space, whose inner product and norm are denoted by $\langle\cdot, \cdot\rangle$ and $\|\cdot\|$, respectively. Let $K$ be a nonempty closed convex subset of $\mathscr{H}$. The (nearest point or metric) projection from $\mathscr{H}$ onto $K$ is denoted by $P_{K}$. We write $x_{n} \rightarrow x$ to indicate that the sequence $\left\{x_{n}\right\}$ converges weakly to $x$ and $x_{n} \rightarrow x$ to indicate that the sequence $\left\{x_{n}\right\}$ converges strongly to $x$.

Let $C$ and $Q$ be nonempty closed convex subsets of infinite-dimensional real Hilbert spaces $\mathscr{H}_{1}$ and $\mathscr{H}_{2}$, respectively. The split feasibility problem (SFP) is to find a point $x^{*}$ with the property:

$$
x^{*} \in C, \quad A x^{*} \in Q,
$$

where $A \in B\left(\mathscr{H}_{1}, \mathscr{H}_{2}\right)$ and $B\left(\mathscr{H}_{1}, \mathscr{H}_{2}\right)$ denotes the family of all bounded linear operators from $\mathscr{H}_{1}$ to $\mathscr{H}_{2}$.

In 1994, the SFP was first introduced by Censor and Elfving [1], in finite-dimensional Hilbert spaces, for modeling inverse problems which arise from phase retrievals and in medical image reconstruction. A number of image reconstruction problems can be formulated as the SFP; see, for example, [2] and the references therein. Recently, it was found that the SFP can also be applied to study intensity-modulated radiation therapy; see, for example, [3-5] and the references therein. In the recent past, a wide variety of iterative methods have been used in signal processing and image reconstruction and for solving the SFP; see, for example, [2-12] and the references therein. A special case of the SFP is the following convex constrained linear inverse problem [13] of finding an element $x$ such that

$$
x \in C, \quad A x=b .
$$

It has been extensively investigated in the literature using the projected Landweber iterative method [14]. Comparatively, the SFP has received much less attention so far, due to the complexity resulting from the set $Q$. Therefore, whether various versions of the projected Landweber iterative method [14] can be extended to solve the SFP remains an interesting 
open topics. For example, it is yet not clear whether the dual approach to (1.2) of [15] can be extended to the SFP. The original algorithm given in [1] involves the computation of the inverse $A^{-1}$ (assuming the existence of the inverse of $A$ ) and thus has not become popular. A seemingly more popular algorithm that solves the SFP is the CQ algorithm of Byrne $[2,7]$ which is found to be a gradient-projection method (GPM) in convex minimization. It is also a special case of the proximal forward-backward splitting method [16]. The CQ algorithm only involves the computation of the projections $P_{C}$ and $P_{Q}$ onto the sets $C$ and $Q$, respectively, and is therefore implementable in the case where $P_{C}$ and $P_{\mathrm{Q}}$ have closed-form expressions, for example, $C$ and $Q$ are closed balls or halfspaces. However, it remains a challenge how to implement the $C Q$ algorithm in the case where the projections $P_{C}$ and/or $P_{Q}$ fail to have closed-form expressions, though theoretically we can prove the (weak) convergence of the algorithm.

Very recently, Xu [6] gave a continuation of the study on the CQ algorithm and its convergence. He applied Mann's algorithm to the SFP and purposed an averaged CQ algorithm which was proved to be weakly convergent to a solution of the SFP. He also established the strong convergence result, which shows that the minimum-norm solution can be obtained.

Furthermore, Korpelevič [17] introduced the so-called extragradient method for finding a solution of a saddle point problem. He proved that the sequences generated by the proposed iterative algorithm converge to a solution of the saddle point problem.

Throughout this paper, assume that the SFP is consistent, that is, the solution set $\Gamma$ of the SFP is nonempty. Let $f$ : $\mathscr{H}_{1} \rightarrow R$ be a continuous differentiable function. The minimization problem

$$
\min _{x \in C} f(x):=\left\|\frac{1}{2} A x-P_{\mathrm{Q}} A x\right\|^{2}
$$

is ill posed. Therefore, $\mathrm{Xu}$ [6] considered the following Tikhonov regularization problem:

$$
\min _{x \in C} f_{\alpha}(x):=\frac{1}{2}\left\|A x-P_{\mathrm{Q}} A x\right\|^{2}+\frac{1}{2} \alpha\|x\|^{2},
$$

where $\alpha>0$ is the regularization parameter. The regularized minimization (4) has a unique solution which is denoted by $x_{\alpha}$. The following results are easy to prove.

Proposition 1 (see [18, Proposition 3.1]). Given $x^{*} \in \mathscr{H}_{1}$, the following statements are equivalent:

(i) $x^{*}$ solves the SFP;

(ii) $x^{*}$ solves the fixed point equation

$$
P_{C}(I-\lambda \nabla f) x^{*}=x^{*}
$$

where $\lambda>0, \nabla f=A^{*}\left(I-P_{\mathrm{Q}}\right) A$ and $A^{*}$ is the adjoint of $A$;

(iii) $x^{*}$ solves the variational inequality problem (VIP) of finding $x^{*} \in C$ such that

$$
\left\langle\nabla f\left(x^{*}\right), x-x^{*}\right\rangle \geq 0, \quad \forall x \in C .
$$

It is clear from Proposition 1 that

$$
\Gamma=\operatorname{Fix}\left(P_{C}(I-\lambda \nabla f)\right)=\operatorname{VI}(C, \nabla f),
$$

for all $\lambda>0$, where $\operatorname{Fix}\left(P_{C}(I-\lambda \nabla f)\right)$ and $\operatorname{VI}(C, \nabla f)$ denote the set of fixed points of $P_{C}(I-\lambda \nabla f)$ and the solution set of VIP (6), respectively.

Proposition 2 (see [18]). There hold the following statements:

(i) the gradient

$$
\nabla f_{\alpha}=\nabla f+\alpha I=A^{*}\left(I-P_{\mathrm{Q}}\right) A+\alpha I
$$

is $\left(\alpha+\|A\|^{2}\right)$-Lipschitz continuous and $\alpha$-strongly monotone;

(ii) the mapping $P_{C}\left(I-\lambda \nabla f_{\alpha}\right)$ is a contraction with coefficient

$$
\sqrt{1-\lambda\left(2 \alpha-\lambda\left(\|A\|^{2}+\alpha\right)^{2}\right)}\left(\leq \sqrt{1-\alpha \lambda} \leq 1-\frac{1}{2} \alpha \lambda\right),
$$

$$
\text { where } 0<\lambda \leq \alpha /\left(\|A\|^{2}+\alpha\right)^{2} \text {; }
$$

(iii) if the SFP is consistent, then the strong $\lim _{\alpha \rightarrow 0} x_{\alpha}$ exists and is the minimum-norm solution of the SFP.

Very recently, by combining the regularization method and extragradient method due to Nadezhkina and Takahashi [19], Ceng et al. [18] proposed an extragradient algorithm with regularization and proved that the sequences generated by the proposed algorithm converge weakly to an element of $\operatorname{Fix}(S) \cap \Gamma$, where $S: C \rightarrow C$ is a nonexpansive mapping.

Theorem 3 (see [18, Theorem 3.1]). Let $S: C \rightarrow C$ be a nonexpansive mapping such that Fix $(S) \cap \Gamma \neq \emptyset$. Let $\left\{x_{n}\right\}$ and $\left\{y_{n}\right\}$ the sequences in $C$ generated by the following extragradient algorithm:

$$
\begin{gathered}
x_{0}=x \in C \text { chosen arbitrarily, } \\
y_{n}=P_{C}\left(x_{n}-\lambda_{n} \nabla f_{\alpha_{n}}\left(x_{n}\right)\right), \\
x_{n+1}=\beta_{n} x_{n}+\left(1-\beta_{n}\right) S P_{C}\left(x_{n}-\lambda_{n} \nabla f_{\alpha_{n}}\left(y_{n}\right)\right), \quad \forall n \geq 0,
\end{gathered}
$$

where $\sum_{n=0}^{\infty} \alpha_{n}<\infty,\left\{\lambda_{n}\right\} \subset[a, b]$ for some $a, b \in\left(0,1 /\|A\|^{2}\right)$ and $\left\{\beta_{n}\right\} \subset[c, d]$ for some $c, d \in(0,1)$. Then, both the sequences $\left\{x_{n}\right\}$ and $\left\{y_{n}\right\}$ converge weakly to an element $\hat{x} \in$ $\operatorname{Fix}(S) \cap \Gamma$.

On the other hand, assume that $C$ is a nonempty closed convex subset of $\mathscr{H}$ and $A: C \rightarrow \mathscr{H}$ is a mapping. The classical variational inequality problem (VIP) is to find $x^{*} \epsilon$ $C$ such that

$$
\left\langle A x^{*}, x-x^{*}\right\rangle \geq 0, \quad \forall x \in C .
$$

It is now well known that the variational inequalities are equivalent to the fixed point problems, the origin of which 
can be traced back to Lions and Stampacchia [20]. This alternative formulation has been used to suggest and analyze projection iterative method for solving variational inequalities under the conditions that the involved operator must be strongly monotone and Lipschitz continuous. Related to the variational inequalities, we have the problem of finding the fixed points of nonexpansive mappings or strict pseudocontraction mappings, which is the current interest in functional analysis. Several people considered a unified approach to solve variational inequality problems and fixed point problems; see, for example, [21-28] and the references therein.

A mapping $A: C \rightarrow \mathscr{H}$ is said to be an $\alpha$-inverse strongly monotone if there exists $\alpha>0$ such that

$$
\langle A x-A y, x-y\rangle \geq \alpha\|A x-A y\|^{2}, \quad \forall x, y \in C .
$$

A mapping $S: C \rightarrow C$ is said to be $k$-strictly pseudocontractive if there exists $0 \leq k<1$ such that

$$
\begin{aligned}
\|S x-S y\|^{2} \leq & \|x-y\|^{2} \\
& +k\|(I-S) x-(I-S) y\|^{2}, \quad \forall x, y \in C .
\end{aligned}
$$

In this case, we also say that $S$ is a $k$-strict pseudo-contraction. In particular, whenever $k=0, S$ becomes a nonexpansive mapping from $C$ into itself. It is clear that every inverse strongly monotone mapping is a monotone and Lipschitz continuous mapping. We denote by $\operatorname{VI}(C, A)$ and $\operatorname{Fix}(S)$ the solution set of problem (11) and the set of all fixed points of $S$, respectively.

For finding an element of $\operatorname{Fix}(S) \cap \operatorname{VI}(C, A)$ under the assumption that a set $C \subset \mathscr{H}$ is nonempty, closed, and convex, a mapping $S: C \rightarrow C$ is nonexpansive, and a mapping $A: C \rightarrow \mathscr{H}$ is $\alpha$-inverse strongly monotone, Takahashi and Toyoda [29] introduced an iterative scheme and studied the weak convergence of the sequence generated by the proposed scheme to a point of $\operatorname{Fix}(S) \cap \operatorname{VI}(C, A)$. Recently, Iiduka and Takahashi [30] presented another iterative scheme for finding an element of $\operatorname{Fix}(S) \cap \operatorname{VI}(C, A)$ and showed that the sequence generated by the scheme converges strongly to $P_{\mathrm{Fix}(S) \cap \mathrm{VI}(C, A)} u$, where $u$ is the initially chosen point in the iterative scheme and $P_{K}$ denotes the metric projection of $\mathscr{H}$ onto $K$.

Based on Korpelevič's extragradient method [17], Nadezhkina and Takahashi [19] introduced an iterative process for finding an element of $\operatorname{Fix}(S) \cap \operatorname{VI}(C, A)$ and proved the weak convergence of the sequence to a point of $\operatorname{Fix}(S) \cap \operatorname{VI}(C, A)$. Zeng and Yao [27] presented an iterative scheme for finding an element of $\operatorname{Fix}(S) \cap \operatorname{VI}(C, A)$ and proved that two sequences generated by the method converges strongly to an element of $\operatorname{Fix}(S) \cap \operatorname{VI}(C, A)$. Recently, Bnouhachem et al. [31] suggested and analyzed an iterative scheme for finding a common element of the fixed point set $\operatorname{Fix}(S)$ of a nonexpansive mapping $S$ and the solution set $\operatorname{VI}(C, A)$ of the variational inequality (11) for an inverse strongly monotone mapping $A: C \rightarrow \mathscr{H}$.

Furthermore, as a much more general generalization of the classical variational inequality problem (11), Ceng et al.
[23] introduced and considered the following problem of finding $\left(x^{*}, y^{*}\right) \in C \times C$ such that

$$
\begin{aligned}
& \left\langle\mu_{1} B_{1} y^{*}+x^{*}-y^{*}, x-x^{*}\right\rangle \geq 0, \quad \forall x \in C, \\
& \left\langle\mu_{2} B_{2} x^{*}+y^{*}-x^{*}, x-y^{*}\right\rangle \geq 0, \quad \forall x \in C,
\end{aligned}
$$

which is called a general system of variational inequalities (GSVI), which $\mu_{1}>0$ and $\mu_{2}>0$ are two constants. The set of solutions of problem (14) is denoted by $\operatorname{GSVI}\left(C, B_{1}, B_{2}\right)$. In particular, if $B_{1}=B_{2}$, then problem (14) reduces to the new system of variational inequalities, introduced and studied by Verma [32]. Recently, Ceng et al. [23] transformed problem (14) into a fixed point problem in the following way.

Lemma 4 (see [23]). For given $\bar{x}, \bar{y} \in C,(\bar{x}, \bar{y})$ is a solution of problem (14) if and only if $\bar{x}$ is a fixed point of the mapping $G: C \rightarrow C$ defined by

$$
\begin{aligned}
G(x)=P_{C} & {\left[P_{C}\left(x-\mu_{2} B_{2} x\right)\right.} \\
- & \left.\mu_{1} B_{1} P_{C}\left(x-\mu_{2} B_{2} x\right)\right], \quad \forall x \in C,
\end{aligned}
$$

where $\bar{y}=P_{C}\left(\bar{x}-\mu_{2} B_{2} \bar{x}\right)$.

In particular, if the mapping $B_{i}: C \rightarrow \mathscr{H}$ is $\beta_{i}$-inverse strongly monotone for $i=1,2$, then the mapping $G$ is nonexpansive provided $\mu_{i} \in\left(0,2 \beta_{i}\right)$ for $i=1,2$.

Utilizing Lemma 4, they introduced and studied a relaxed extragradient method for solving problem (14). Throughout this paper, the set of fixed points of the mapping $G$ is denoted by $\Xi$. Based on the relaxed extragradient method and viscosity approximation method, Yao et al. [26] proposed and analyzed an iterative algorithm for finding a common solution of the GSVI (14) and the fixed point problem of a strictly pseudo-contractive mapping $S: C \rightarrow C$. Subsequently, Ceng et al. [33] further presented and analyzed an iterative scheme for finding a common element of the solution set of the VIP (11), the solution set of the GSVI (14), and the fixed point set of a strictly pseudo-contractive mapping $S: C \rightarrow C$.

Theorem 5 (see [33, Theorem 3.1]). Let $C$ be a nonempty closed convex subset of a real Hilbert space $\mathscr{H}$. Let $A: C \rightarrow \mathscr{H}$ be $\alpha$-inverse strongly monotone and $B_{i}: C \rightarrow \mathscr{H}$ be $\beta_{i}$-inverse strongly monotone for $i=1,2$. Let $S: C \rightarrow C$ be a $k$-strictly pseudo-contractive mapping such that $\operatorname{Fix}(S) \cap \Xi \cap V I(C, A) \neq \emptyset$. Let $Q: C \rightarrow C$ be a $\rho$-contraction with $\rho \in[0,1 / 2)$. For given $x_{0} \in C$ arbitrarily, let the sequences $\left\{x_{n}\right\},\left\{u_{n}\right\},\left\{\widetilde{u}_{n}\right\}$ be generated by the relaxed extragradient iterative scheme:

$$
\begin{gathered}
u_{n}=P_{C}\left[P_{C}\left(x_{n}-\mu_{2} B_{2} x_{n}\right)-\mu_{1} B_{1} P_{C}\left(x_{n}-\mu_{2} B_{2} x_{n}\right)\right], \\
\tilde{u}_{n}=P_{C}\left(u_{n}-\lambda_{n} A u_{n}\right), \\
y_{n}=\alpha_{n} Q x_{n}+\left(1-\alpha_{n}\right) P_{C}\left(u_{n}-\lambda_{n} A \tilde{u}_{n}\right), \\
x_{n+1}=\beta_{n} x_{n}+\gamma_{n} y_{n}+\delta_{n} S y_{n}, \quad \forall n \geq 0,
\end{gathered}
$$

where $\mu_{i} \in\left(0,2 \beta_{i}\right)$ for $i=1,2$, and the following conditions hold for five sequences $\left\{\lambda_{n}\right\} \subset(0, \alpha]$ and $\left\{\alpha_{n}\right\},\left\{\beta_{n}\right\},\left\{\gamma_{n}\right\},\left\{\delta_{n}\right\} \subset$ $[0,1]$ : 
(i) $\beta_{n}+\gamma_{n}+\delta_{n}=1$ and $\left(\gamma_{n}+\delta_{n}\right) k \leq \gamma_{n}$ for all $n \geq 0$;

(ii) $\lim _{n \rightarrow \infty} \alpha_{n}=0$ and $\sum_{n=0}^{\infty} \alpha_{n}=\infty$;

(iii) $0<\liminf _{n \rightarrow \infty} \beta_{n} \leq \limsup _{n \rightarrow \infty} \beta_{n}<1$ and $\liminf _{n \rightarrow \infty} \delta_{n}>0$;

(iv) $\lim _{n \rightarrow \infty}\left(\gamma_{n+1} /\left(1-\beta_{n+1}\right)-\gamma_{n} /\left(1-\beta_{n}\right)\right)=0$;

(v) $0<\liminf _{n \rightarrow \infty} \lambda_{n} \leq \limsup _{n \rightarrow \infty} \lambda_{n}<\alpha$ and $\lim _{n \rightarrow \infty}\left|\lambda_{n+1}-\lambda_{n}\right|=0$.

Then the sequences $\left\{x_{n}\right\},\left\{u_{n}\right\},\left\{\tilde{u}_{n}\right\}$ converge strongly to the same point $\bar{x}=P_{\operatorname{Fix}(S) \cap \Xi \cap V I(C, A)} Q \bar{x}$ if and only if $\lim _{n \rightarrow \infty}$ $\left\|u_{n+1}-u_{n}\right\|=0$. Furthermore, $(\bar{x}, \bar{y})$ is a solution of the GSVI (14), where $\bar{y}=P_{C}\left(\bar{x}-\mu_{2} B_{2} \bar{x}\right)$.

Motivated and inspired by the research going on this area, we propose and analyze the following relaxed extragradient iterative algorithms with regularization for finding a common element of the solution set of the GSVI (14), the solution set of the SFP (1), and the fixed point set of a strictly pseudocontractive mapping $S: C \rightarrow C$.

Algorithm 6. Let $\mu_{i} \in\left(0,2 \beta_{i}\right)$ for $i=1,2,\left\{\alpha_{n}\right\} \subset(0, \infty)$, $\left\{\lambda_{n}\right\} \subset\left(0,1 /\|A\|^{2}\right)$ and $\left\{\sigma_{n}\right\},\left\{\beta_{n}\right\},\left\{\gamma_{n}\right\},\left\{\delta_{n}\right\} \subset[0,1]$ such that $\beta_{n}+\gamma_{n}+\delta_{n}=1$ for all $n \geq 0$. For given $x_{0} \in C$ arbitrarily, let $\left\{x_{n}\right\},\left\{u_{n}\right\},\left\{\widetilde{u}_{n}\right\}$ be the sequences generated by the following relaxed extragradient iterative scheme with regularization:

$$
\begin{gathered}
u_{n}=P_{C}\left[P_{C}\left(x_{n}-\mu_{2} B_{2} x_{n}\right)-\mu_{1} B_{1} P_{C}\left(x_{n}-\mu_{2} B_{2} x_{n}\right)\right], \\
\tilde{u}_{n}=P_{C}\left(u_{n}-\lambda_{n} \nabla f_{\alpha_{n}}\left(u_{n}\right)\right), \\
y_{n}=\sigma_{n} Q x_{n}+\left(1-\sigma_{n}\right) P_{C}\left(u_{n}-\lambda_{n} \nabla f_{\alpha_{n}}\left(\tilde{u}_{n}\right)\right), \\
x_{n+1}=\beta_{n} x_{n}+\gamma_{n} y_{n}+\delta_{n} S y_{n}, \quad \forall n \geq 0 .
\end{gathered}
$$

Under mild assumptions, it is proven that the sequences $\left\{x_{n}\right\},\left\{u_{n}\right\},\left\{\tilde{u}_{n}\right\}$ converge strongly to the same point $\bar{x}=$ $P_{\text {Fix }(S) \cap \Xi \cap \Gamma} Q \bar{x}$ if and only if $\lim _{n \rightarrow \infty}\left\|u_{n+1}-u_{n}\right\|=0$. Furthermore, $(\bar{x}, \bar{y})$ is a solution of the GSVI (14), where $\bar{y}=P_{C}\left(\bar{x}-\mu_{2} B_{2} \bar{x}\right)$.

Algorithm 7. Let $\mu_{i} \in\left(0,2 \beta_{i}\right)$ for $i=1,2,\left\{\alpha_{n}\right\} \subset(0, \infty)$, $\left\{\lambda_{n}\right\} \subset\left(0,1 /\|A\|^{2}\right)$ and $\left\{\sigma_{n}\right\},\left\{\tau_{n}\right\},\left\{\beta_{n}\right\},\left\{\gamma_{n}\right\},\left\{\delta_{n}\right\} \subset[0,1]$ such that $\sigma_{n}+\tau_{n} \leq 1$ and $\beta_{n}+\gamma_{n}+\delta_{n}=1$ for all $n \geq 0$. For given $x_{0} \in$ $C$ arbitrarily, let $\left\{x_{n}\right\},\left\{y_{n}\right\},\left\{z_{n}\right\}$ be the sequences generated by the following relaxed extragradient iterative scheme with regularization:

$$
\begin{aligned}
z_{n}= & P_{C}\left(x_{n}-\lambda_{n} \nabla f_{\alpha_{n}}\left(x_{n}\right)\right), y_{n} \\
= & \sigma_{n} Q x_{n}+\tau_{n} P_{C}\left(x_{n}-\lambda_{n} \nabla f_{\alpha_{n}}\left(z_{n}\right)\right) \\
& +\left(1-\sigma_{n}-\tau_{n}\right) P_{C}\left[P_{C}\left(z_{n}-\mu_{2} B_{2} z_{n}\right)\right. \\
& \left.-\mu_{1} B_{1} P_{C}\left(z_{n}-\mu_{2} B_{2} z_{n}\right)\right], x_{n+1} \\
= & \beta_{n} x_{n}+\gamma_{n} y_{n}+\delta_{n} S y_{n}, \quad \forall n \geq 0,
\end{aligned}
$$

Also, under appropriate conditions, it is shown that the sequences $\left\{x_{n}\right\},\left\{y_{n}\right\},\left\{z_{n}\right\}$ converge strongly to the same point
$\bar{x}=P_{\text {Fix }(S) \cap E \cap \Gamma} Q \bar{x}$ if and only if $\lim _{n \rightarrow \infty}\left\|z_{n+1}-z_{n}\right\|=0$. Furthermore, $(\bar{x}, \bar{y})$ is a solution of the GSVI (14), where $\bar{y}=P_{C}\left(\bar{x}-\mu_{2} B_{2} \bar{x}\right)$.

Note that both [6, Theorem 5.7] and [18, Theorem 3.1] are weak convergence results for solving the SFP (1). Beyond question our strong convergence results are very interesting and quite valuable. Because our relaxed extragradient iterative schemes (17) and (18) with regularization involve a contractive self-mapping $Q$, a $k$-strictly pseudo-contractive self-mapping $S$ and several parameter sequences, they are more flexible and more subtle than the corresponding ones in [6, Theorem 5.7] and [18, Theorem 3.1], respectively. Furthermore, the relaxed extragradient iterative scheme (16) is extended to develop our relaxed extragradient iterative schemes (17) and (18) with regularization. All in all, our results represent the modification, supplementation, extension, and improvement of [6, Theorem 5.7], [18, Theorem 3.1], and [33, Theorem 3.1].

\section{Preliminaries}

Let $K$ be a nonempty, closed, and convex subset of a real Hilbert space $\mathscr{H}$. Now we present some known results and definitions which will be used in the sequel.

The metric (or nearest point) projection from $\mathscr{H}$ onto $K$ is the mapping $P_{K}: \mathscr{H} \rightarrow K$ which assigns to each point $x \in \mathscr{H}$ the unique point $P_{K} x \in K$ satisfying the property

$$
\left\|x-P_{K} x\right\|=\inf _{y \in K}\|x-y\|=: d(x, K) .
$$

The following properties of projections are useful and pertinent to our purpose.

Proposition 8 (see [34]). For given $x \in \mathscr{H}$ and $z \in K$ :

(i) $z=P_{K} x \Leftrightarrow\langle x-z, y-z\rangle \leq 0, \forall y \in K$;

(ii) $z=P_{K} x \Leftrightarrow\|x-z\|^{2} \leq\|x-y\|^{2}-\|y-z\|^{2}, \forall y \in K$;

(iii) $\left\langle P_{K} x-P_{K} y, x-y\right\rangle \geq\left\|P_{K} x-P_{K} y\right\|^{2}, \forall y \in \mathscr{H}$, which hence implies that $P_{K}$ is nonexpansive and monotone.

Definition 9. A mapping $T: \mathscr{H} \rightarrow \mathscr{H}$ is said to be

(a) nonexpansive if

$$
\|T x-T y\| \leq\|x-y\|, \quad \forall x, y \in \mathscr{H} ;
$$

(b) firmly nonexpansive if $2 T-I$ is nonexpansive, or equivalently,

$$
\langle x-y, T x-T y\rangle \geq\|T x-T y\|^{2}, \quad \forall x, y \in \mathscr{H} ;
$$

alternatively, $T$ is firmly nonexpansive if and only if $T$ can be expressed as

$$
T=\frac{1}{2}(I+S),
$$

where $S: \mathscr{H} \rightarrow \mathscr{H}$ is nonexpansive; projections are firmly nonexpansive. 
Definition 10. Let $T$ be a nonlinear operator with domain $D(T) \subseteq \mathscr{H}$ and range $R(T) \subseteq \mathscr{H}$.

(a) $T$ is said to be monotone if

$$
\langle x-y, T x-T y\rangle \geq 0, \quad \forall x, y \in D(T) .
$$

(b) Given a number $\beta>0, T$ is said to be $\beta$-strongly monotone if

$\langle x-y, T x-T y\rangle \geq \beta\|x-y\|^{2}, \quad \forall x, y \in D(T)$.

(c) Given a number $v>0, T$ is said to be $v$-inverse strongly monotone ( $\nu$-ism) if

$$
\langle x-y, T x-T y\rangle \geq v\|T x-T y\|^{2}, \quad \forall x, y \in D(T) .
$$

It can be easily seen that if $S$ is nonexpansive, then $I-S$ is monotone. It is also easy to see that a projection $P_{K}$ is 1 -ism.

Inverse strongly monotone (also referred to as cocoercive) operators have been applied widely in solving practical problems in various fields, for instance, in traffic assignment problems; see, for example, [35, 36].

Definition 11. A mapping $T: \mathscr{H} \rightarrow \mathscr{H}$ is said to be an averaged mapping if it can be written as the average of the identity $I$ and a nonexpansive mapping, that is,

$$
T \equiv(1-\alpha) I+\alpha S
$$

where $\alpha \in(0,1)$ and $S: \mathscr{H} \rightarrow \mathscr{H}$ is nonexpansive. More precisely, when the last equality holds, we say that $T$ is $\alpha$ averaged. Thus firmly nonexpansive mappings (in particular, projections) are 1/2-averaged maps.

Proposition 12 (see [7]). Let $T: \mathscr{H} \rightarrow \mathscr{H}$ be a given mapping.

(i) $T$ is nonexpansive if and only if the complement $I-T$ is $1 / 2$-ism.

(ii) If $T$ is $\nu$-ism, then for $\gamma>0, \gamma T$ is $\nu / \gamma$-ism.

(iii) $T$ is averaged if and only if the complement $I-T$ is $\nu$-ism for some $v>1 / 2$. Indeed, for $\alpha \in(0,1), T$ is $\alpha$-averaged if and only if $I-T$ is $1 / 2 \alpha$-ism.

Proposition 13 (see $[7,37]$ ). Let $S, T, V: \mathscr{H} \rightarrow \mathscr{H}$ be given operators.

(i) If $T=(1-\alpha) S+\alpha V$ for some $\alpha \in(0,1)$ and if $S$ is averaged and $V$ is nonexpansive, then $T$ is averaged.

(ii) $T$ is firmly nonexpansive if and only if the complement $I-T$ is firmly nonexpansive.

(iii) If $T=(1-\alpha) S+\alpha V$ for some $\alpha \in(0,1)$ and if $S$ is firmly nonexpansive and $V$ is nonexpansive, then $T$ is averaged.

(iv) The composite of finitely many averaged mappings is averaged. That is, if each of the mappings $\left\{T_{i}\right\}_{i=1}^{N}$ is averaged, then so is the composite $T_{1} \circ T_{2} \circ \cdots \circ T_{N}$. In particular, if $T_{1}$ is $\alpha_{1}$-averaged and $T_{2}$ is $\alpha_{2}$-averaged, where $\alpha_{1}, \alpha_{2} \in(0,1)$, then the composite $T_{1} \circ T_{2}$ is $\alpha$ averaged, where $\alpha=\alpha_{1}+\alpha_{2}-\alpha_{1} \alpha_{2}$. (v) If the mappings $\left\{T_{i}\right\}_{i=1}^{N}$ are averaged and have a common fixed point, then

$$
\bigcap_{i=1}^{N} \operatorname{Fix}\left(T_{i}\right)=\operatorname{Fix}\left(T_{1} \cdots T_{N}\right) \text {. }
$$

The notation $\operatorname{Fix}(T)$ denotes the set of all fixed points of the mapping $T$, that is, $\operatorname{Fix}(T)=\{x \in \mathscr{H}: T x=x\}$.

It is clear that, in a real Hilbert space $\mathscr{H}, S: C \rightarrow C$ is $k$-strictly pseudo-contractive if and only if there holds the following inequality:

$$
\begin{aligned}
& \langle S x-S y, x-y\rangle \\
& \quad \leq\|x-y\|^{2}-\frac{1-k}{2}\|(I-S) x-(I-S) y\|^{2}, \quad \forall x, y \in C .
\end{aligned}
$$

This immediately implies that if $S$ is a $k$-strictly pseudocontractive mapping, then $I-S$ is $(1-k) / 2$-inverse strongly monotone; for further detail, we refer to [38] and the references therein. It is well known that the class of strict pseudo-contractions strictly includes the class of nonexpansive mappings.

In order to prove the main result of this paper, the following lemmas will be required.

Lemma 14 (see [39]). Let $\left\{x_{n}\right\}$ and $\left\{y_{n}\right\}$ be bounded sequences in a Banach space $X$ and let $\left\{\beta_{n}\right\}$ be a sequence in $[0,1]$ with $0<\liminf _{n \rightarrow \infty} \beta_{n} \leq \limsup _{n \rightarrow \infty} \beta_{n}<1$. Suppose $x_{n+1}=$ $\left(1-\beta_{n}\right) y_{n}+\beta_{n} x_{n}$ for all integers $n \geq 0$ and $\lim \sup _{n \rightarrow \infty}\left(\| y_{n+1}-\right.$ $\left.y_{n}\|-\| x_{n+1}-x_{n} \|\right) \leq 0$. Then, $\lim _{n \rightarrow \infty}\left\|y_{n}-x_{n}\right\|=0$.

Lemma 15 (see [38, Proposition 2.1]). Let $C$ be a nonempty closed convex subset of a real Hilbert space $\mathscr{H}$ and $S: C \rightarrow C$ a mapping.

(i) If $S$ is a $k$-strict pseudo-contractive mapping, then $S$ satisfies the Lipschitz condition

$$
\|S x-S y\| \leq \frac{1+k}{1-k}\|x-y\|, \quad \forall x, y \in C .
$$

(ii) If $S$ is a $k$-strict pseudo-contractive mapping, then the mapping $I-S$ is semiclosed at 0 , that is, if $\left\{x_{n}\right\}$ is a sequence in $C$ such that $x_{n} \rightarrow \tilde{x}$ weakly and (I$S) x_{n} \rightarrow 0$ strongly, then $(I-S) \tilde{x}=0$.

(iii) If $S$ is $k$-(quasi-)strict pseudo-contraction, then the fixed point set Fix $(S)$ of $S$ is closed and convex so that the projection $P_{\text {Fix (S) }}$ is well defined.

The following lemma plays a key role in proving strong convergence of the sequences generated by our algorithms.

Lemma 16 (see [34]). Let $\left\{a_{n}\right\}$ be a sequence of nonnegative real numbers satisfying the property

$$
a_{n+1} \leq\left(1-s_{n}\right) a_{n}+s_{n} t_{n}+r_{n}, \quad \forall n \geq 0,
$$

where $\left\{s_{n}\right\} \subset(0,1]$ and $\left\{t_{n}\right\}$ are such that 
(i) $\sum_{n=0}^{\infty} s_{n}=\infty$;

(ii) either $\lim \sup _{n \rightarrow \infty} t_{n} \leq 0$ or $\sum_{n=0}^{\infty}\left|s_{n} t_{n}\right|<\infty$;

(iii) $\sum_{n=0}^{\infty} r_{n}<\infty$ where $r_{n} \geq 0, \forall n \geq 0$. Then, $\lim _{n \rightarrow \infty} a_{n}=0$.

Lemma 17 (see [26]). Let $C$ be a nonempty closed convex subset of a real Hilbert space $\mathscr{H}$. Let $S: C \rightarrow C$ be a $k$-strictly pseudo-contractive mapping. Let $\gamma$ and $\delta$ be two nonnegative real numbers such that $(\gamma+\delta) k \leq \gamma$. Then

$$
\|\gamma(x-y)+\delta(S x-S y)\| \leq(\gamma+\delta)\|x-y\|, \quad \forall x, y \in C .
$$

The following lemma is an immediate consequence of an inner product.

Lemma 18. In a real Hilbert space $\mathscr{H}$, there holds the inequality

$$
\|x+y\|^{2} \leq\|x\|^{2}+2\langle y, x+y\rangle, \quad \forall x, y \in \mathscr{H} .
$$

Let $K$ be a nonempty closed convex subset of a real Hilbert space $\mathscr{H}$ and let $F: K \rightarrow \mathscr{H}$ be a monotone mapping. The variational inequality problem (VIP) is to find $x \in K$ such that

$$
\langle F x, y-x\rangle \geq 0, \quad \forall y \in K
$$

The solution set of the VIP is denoted by $\mathrm{VI}(K, F)$. It is well known that

$$
x \in \mathrm{VI}(K, F) \Longleftrightarrow x=P_{K}(x-\lambda F x), \quad \forall \lambda>0 .
$$

A set-valued mapping $T: \mathscr{H} \rightarrow 2^{\mathscr{H}}$ is called monotone if for all $x, y \in \mathscr{H}, f \in T x$ and $g \in T y$ imply that $\langle x-y, f-$ $g\rangle \geq 0$. A monotone set-valued mapping $T: \mathscr{H} \rightarrow 2^{\mathscr{H}}$ is called maximal if its graph $\mathrm{Gph}(T)$ is not properly contained in the graph of any other monotone set-valued mapping. It is known that a monotone set-valued mapping $T: \mathscr{H} \rightarrow 2^{\mathscr{H}}$ is maximal if and only if for $(x, f) \in \mathscr{H} \times \mathscr{H},\langle x-y, f-g\rangle \geq 0$ for every $(y, g) \in \mathrm{Gph}(T)$ implies that $f \in T x$. Let $F: K \rightarrow$ $\mathscr{H}$ be a monotone and Lipschitz continuous mapping and let $N_{K} v$ be the normal cone to $K$ at $v \in K$, that is,

$$
N_{K} v=\{w \in \mathscr{H}:\langle v-u, w\rangle \geq 0, \forall u \in K\} .
$$

Define

$$
T v=\left\{\begin{array}{cl}
F v+N_{K} v, & \text { if } v \in K, \\
\emptyset, & \text { if } v \notin K .
\end{array}\right.
$$

It is known that in this case the mapping $T$ is maximal monotone, and $0 \in T v$ if and only if $v \in \operatorname{VI}(K, F)$; see further details, one refers to [40] and the references therein.

\section{Main Results}

In this section, we first prove the strong convergence of the sequences generated by the relaxed extragradient iterative algorithm (17) with regularization.
Theorem 19. Let $C$ be a nonempty closed convex subset of a real Hilbert space $\mathscr{H}_{1}$. Let $A \in B\left(\mathscr{H}_{1}, \mathscr{H}_{2}\right)$, and let $B_{i}: C \rightarrow$ $\mathscr{H}_{1}$ be $\beta_{i}$-inverse strongly monotone for $i=1,2$. Let $S: C \rightarrow C$ be a $k$-strictly pseudo-contractive mapping such that $\operatorname{Fix}(S) \cap$ $\Xi \cap \Gamma \neq \emptyset$. Let $Q: C \rightarrow C$ be a $\rho$-contraction with $\rho \in[0,1 / 2)$. For given $x_{0} \in C$ arbitrarily, let the sequences $\left\{x_{n}\right\},\left\{u_{n}\right\},\left\{\tilde{u}_{n}\right\}$ be generated by the relaxed extragradient iterative algorithm (17) with regularization, where $\mu_{i} \in\left(0,2 \beta_{i}\right)$ for $i=1,2,\left\{\alpha_{n}\right\} \subset$ $(0, \infty),\left\{\lambda_{n}\right\} \subset\left(0,1 /\|A\|^{2}\right)$ and $\left\{\sigma_{n}\right\},\left\{\beta_{n}\right\},\left\{\gamma_{n}\right\},\left\{\delta_{n}\right\} \subset[0,1]$ such that

(i) $\sum_{n=0}^{\infty} \alpha_{n}<\infty$;

(ii) $\beta_{n}+\gamma_{n}+\delta_{n}=1$ and $\left(\gamma_{n}+\delta_{n}\right) k \leq \gamma_{n}$ for all $n \geq 0$;

(iii) $\lim _{n \rightarrow \infty} \sigma_{n}=0$ and $\sum_{n=0}^{\infty} \sigma_{n}=\infty$;

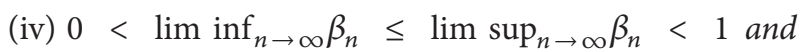
$\liminf _{n \rightarrow \infty} \delta_{n}>0$;

(v) $\lim _{n \rightarrow \infty}\left(\gamma_{n+1} /\left(1-\beta_{n+1}\right)-\gamma_{n} /\left(1-\beta_{n}\right)\right)=0$;

(vi) $0<\liminf _{n \rightarrow \infty} \lambda_{n} \leq \lim \sup _{n \rightarrow \infty} \lambda_{n}<1 /\|A\|^{2}$ and $\lim _{n \rightarrow \infty}\left|\lambda_{n+1}-\lambda_{n}\right|=0$.

Then the sequences $\left\{x_{n}\right\},\left\{u_{n}\right\},\left\{\tilde{u}_{n}\right\}$ converge strongly to the same point $\bar{x}=P_{\mathrm{Fix}(S) \cap \Xi \cap \Gamma} Q \bar{x}$ if and only if $\lim _{n \rightarrow \infty} \| u_{n+1}-$ $u_{n} \|=0$. Furthermore, $(\bar{x}, \bar{y})$ is a solution of the GSVI (14), where $\bar{y}=P_{C}\left(\bar{x}-\mu_{2} B_{2} \bar{x}\right)$.

Proof. First, taking into account $0<\liminf _{n \rightarrow \infty} \lambda_{n} \leq$ $\limsup _{n \rightarrow \infty} \lambda_{n}<1 /\|A\|^{2}$, without loss of generality we may assume that $\left\{\lambda_{n}\right\} \subset[a, b]$ for some $a, b \in\left(0,1 /\|A\|^{2}\right)$.

Now, let us show that $P_{C}\left(I-\lambda \nabla f_{\alpha}\right)$ is $\zeta$-averaged for each $\lambda \in\left(0,2 /\left(\alpha+\|A\|^{2}\right)\right)$, where

$$
\zeta=\frac{2+\lambda\left(\alpha+\|A\|^{2}\right)}{4} .
$$

Indeed, it is easy to see that $\nabla f=A^{*}\left(I-P_{\mathrm{Q}}\right) A$ is $1 /\|A\|^{2}$ ism, that is,

$$
\langle\nabla f(x)-\nabla f(y), x-y\rangle \geq \frac{1}{\|A\|^{2}}\|\nabla f(x)-\nabla f(y)\|^{2} .
$$

Observe that

$$
\begin{aligned}
(\alpha+ & \left.\|A\|^{2}\right)\left\langle\nabla f_{\alpha}(x)-\nabla f_{\alpha}(y), x-y\right\rangle \\
= & \left(\alpha+\|A\|^{2}\right)\left[\alpha\|x-y\|^{2}\right. \\
& +\langle\nabla f(x)-\nabla f(y), x-y\rangle] \\
= & \alpha^{2}\|x-y\|^{2} \\
& +\alpha\langle\nabla f(x)-\nabla f(y), x-y\rangle+\alpha\|A\|^{2}\|x-y\|^{2} \\
& +\|A\|^{2}\langle\nabla f(x)-\nabla f(y), x-y\rangle \\
\geq & \alpha^{2}\|x-y\|^{2}+2 \alpha\langle\nabla f(x)-\nabla f(y), x-y\rangle \\
& +\|\nabla f(x)-\nabla f(y)\|^{2} \\
= & \|\alpha(x-y)+\nabla f(x)-\nabla f(y)\|^{2} \\
= & \left\|\nabla f_{\alpha}(x)-\nabla f_{\alpha}(y)\right\|^{2} .
\end{aligned}
$$


Hence, it follows that $\nabla f_{\alpha}=\alpha I+A^{*}\left(I-P_{\mathrm{Q}}\right) A$ is $1 /(\alpha+$ $\left.\|A\|^{2}\right)$-ism. Thus, $\lambda \nabla f_{\alpha}$ is $1 / \lambda\left(\alpha+\|A\|^{2}\right)$-ism according to Proposition 12(ii). By Proposition 12(iii) the complement $I-$ $\lambda \nabla f_{\alpha}$ is $\lambda\left(\alpha+\|A\|^{2}\right) / 2$-averaged. Therefore, noting that $P_{C}$ is $1 / 2$-averaged, and utilizing Proposition 13(iv), we know that for each $\lambda \in\left(0,2 /\left(\alpha+\|A\|^{2}\right)\right), P_{C}\left(I-\lambda \nabla f_{\alpha}\right)$ is $\zeta$-averaged with

$$
\begin{aligned}
\zeta & =\frac{1}{2}+\frac{\lambda\left(\alpha+\|A\|^{2}\right)}{2}-\frac{1}{2} \cdot \frac{\lambda\left(\alpha+\|A\|^{2}\right)}{2} \\
& =\frac{2+\lambda\left(\alpha+\|A\|^{2}\right)}{4} \in(0,1) .
\end{aligned}
$$

This shows that $P_{C}\left(I-\lambda \nabla f_{\alpha}\right)$ is nonexpansive. Furthermore, for $\left\{\lambda_{n}\right\} \subset[a, b]$ with $a, b \in\left(0,1 /\|A\|^{2}\right)$, we have

$$
a \leq \inf _{n \geq 0} \lambda_{n} \leq \sup _{n \geq 0} \lambda_{n} \leq b<\frac{1}{\|A\|^{2}}=\lim _{n \rightarrow \infty} \frac{1}{\alpha_{n}+\|A\|^{2}} .
$$

Without loss of generality we may assume that

$$
a \leq \inf _{n \geq 0} \lambda_{n} \leq \sup _{n \geq 0} \lambda_{n} \leq b<\frac{1}{\alpha_{n}+\|A\|^{2}}, \quad \forall n \geq 0 .
$$

Consequently, it follows that for each integer $n \geq 0, P_{C}(I-$ $\left.\lambda_{n} \nabla f_{\alpha_{n}}\right)$ is $\zeta_{n}$-averaged with

$$
\begin{aligned}
\zeta_{n} & =\frac{1}{2}+\frac{\lambda_{n}\left(\alpha_{n}+\|A\|^{2}\right)}{2}-\frac{1}{2} \cdot \frac{\lambda_{n}\left(\alpha_{n}+\|A\|^{2}\right)}{2} \\
& =\frac{2+\lambda_{n}\left(\alpha_{n}+\|A\|^{2}\right)}{4} \in(0,1) .
\end{aligned}
$$

This immediately implies that $P_{C}\left(I-\lambda_{n} \nabla f_{\alpha_{n}}\right)$ is nonexpansive for all $n \geq 0$.

Next we divide the remainder of the proof into several steps.

Step $1 .\left\{x_{n}\right\}$ is bounded.

Indeed, take an arbitrary $p \in \operatorname{Fix}(S) \cap \Xi \cap \Gamma$. Then, we get $S p=p, P_{C}(I-\lambda \nabla f) p=p$ for $\lambda \in\left(0,2 /\|A\|^{2}\right)$, and

$$
p=P_{C}\left[P_{C}\left(p-\mu_{2} B_{2} p\right)-\mu_{1} B_{1} P_{C}\left(p-\mu_{2} B_{2} p\right)\right] .
$$

From (17) it follows that

$$
\begin{aligned}
\left\|\widetilde{u}_{n}-p\right\|= & \left\|P_{C}\left(I-\lambda_{n} \nabla f_{\alpha_{n}}\right) u_{n}-P_{C}\left(I-\lambda_{n} \nabla f\right) p\right\| \\
\leq & \left\|P_{C}\left(I-\lambda_{n} \nabla f_{\alpha_{n}}\right) u_{n}-P_{C}\left(I-\lambda_{n} \nabla f_{\alpha_{n}}\right) p\right\| \\
& +\left\|P_{C}\left(I-\lambda_{n} \nabla f_{\alpha_{n}}\right) p-P_{C}\left(I-\lambda_{n} \nabla f\right) p\right\| \\
\leq & \left\|u_{n}-p\right\|+\left\|\left(I-\lambda_{n} \nabla f_{\alpha_{n}}\right) p-\left(I-\lambda_{n} \nabla f\right) p\right\| \\
\leq & \left\|u_{n}-p\right\|+\lambda_{n} \alpha_{n}\|p\| .
\end{aligned}
$$

Utilizing Lemma 18 we also have

$$
\begin{aligned}
\left\|\tilde{u}_{n}-p\right\|^{2}= & \left\|P_{C}\left(I-\lambda_{n} \nabla f_{\alpha_{n}}\right) u_{n}-P_{C}\left(I-\lambda_{n} \nabla f\right) p\right\|^{2} \\
= & \| P_{C}\left(I-\lambda_{n} \nabla f_{\alpha_{n}}\right) u_{n}-P_{C}\left(I-\lambda_{n} \nabla f_{\alpha_{n}}\right) p \\
& +P_{C}\left(I-\lambda_{n} \nabla f_{\alpha_{n}}\right) p-P_{C}\left(I-\lambda_{n} \nabla f\right) p \|^{2} \\
\leq & \left\|P_{C}\left(I-\lambda_{n} \nabla f_{\alpha_{n}}\right) u_{n}-P_{C}\left(I-\lambda_{n} \nabla f_{\alpha_{n}}\right) p\right\|^{2} \\
& +2\left\langle P_{C}\left(I-\lambda_{n} \nabla f_{\alpha_{n}}\right) p\right. \\
\left.-P_{C}\left(I-\lambda_{n} \nabla f\right) p, \widetilde{u}_{n}-p\right\rangle & \quad-P_{C}\left(I-\lambda_{n} \nabla f\right) p\|\| \tilde{u}_{n}-p \| \\
\leq & \left\|u_{n}-p\right\|^{2}+2 \| P_{C}\left(I-\lambda_{n} \nabla f_{\alpha_{n}}\right) p \\
\leq & \left\|u_{n}-p\right\|^{2}+2 \|\left(I-\lambda_{n} \nabla f_{\alpha_{n}}\right) p \\
\quad & \quad\left(I-\lambda_{n} \nabla f\right) p\|\| \tilde{u}_{n}-p \| \\
= & \left\|u_{n}-p\right\|^{2}+2 \lambda_{n} \alpha_{n}\|p\|\left\|\tilde{u}_{n}-p\right\| .
\end{aligned}
$$

For simplicity, we write

$$
\begin{aligned}
& q=P_{C}\left(p-\mu_{2} B_{2} p\right), \quad \tilde{x}_{n}=P_{C}\left(x_{n}-\mu_{2} B_{2} x_{n}\right), \\
& \bar{u}_{n}=P_{C}\left(u_{n}-\lambda_{n} \nabla f_{\alpha_{n}}\left(\widetilde{u}_{n}\right)\right),
\end{aligned}
$$

for each $n \geq 0$. Then $y_{n}=\sigma_{n} Q x_{n}+\left(1-\sigma_{n}\right) \bar{u}_{n}$ for each $n \geq 0$. Since $B_{i}: C \rightarrow \mathscr{H}_{1}$ is $\beta_{i}$-inverse strongly monotone for $i=$ 1,2 and $0<\mu_{i}<2 \beta_{i}$ for $i=1,2$, we know that for all $n \geq 0$,

$$
\begin{gathered}
\left\|u_{n}-p\right\|^{2} \\
=\| P_{C}\left[P_{C}\left(x_{n}-\mu_{2} B_{2} x_{n}\right)\right. \\
\left.\quad-\mu_{1} B_{1} P_{C}\left(x_{n}-\mu_{2} B_{2} x_{n}\right)\right]-p \|^{2} \\
=\| P_{C}\left[P_{C}\left(x_{n}-\mu_{2} B_{2} x_{n}\right)-\mu_{1} B_{1} P_{C}\left(x_{n}-\mu_{2} B_{2} x_{n}\right)\right] \\
\quad-P_{C}\left[P_{C}\left(p-\mu_{2} B_{2} p\right)-\mu_{1} B_{1} P_{C}\left(p-\mu_{2} B_{2} p\right)\right] \|^{2} \\
\leq \|\left[P_{C}\left(x_{n}-\mu_{2} B_{2} x_{n}\right)-\mu_{1} B_{1} P_{C}\left(x_{n}-\mu_{2} B_{2} x_{n}\right)\right] \\
\quad-\left[P_{C}\left(p-\mu_{2} B_{2} p\right)-\mu_{1} B_{1} P_{C}\left(p-\mu_{2} B_{2} p\right)\right] \|^{2} \\
=\|\left[P_{C}\left(x_{n}-\mu_{2} B_{2} x_{n}\right)-P_{C}\left(p-\mu_{2} B_{2} p\right)\right] \\
\quad-\mu_{1}\left[B_{1} P_{C}\left(x_{n}-\mu_{2} B_{2} x_{n}\right)-B_{1} P_{C}\left(p-\mu_{2} B_{2} p\right)\right] \|^{2} \\
\leq\left\|P_{C}\left(x_{n}-\mu_{2} B_{2} x_{n}\right)-P_{C}\left(p-\mu_{2} B_{2} p\right)\right\|^{2} \\
-\mu_{1}\left(2 \beta_{1}-\mu_{1}\right) \| B_{1} P_{C}\left(x_{n}-\mu_{2} B_{2} x_{n}\right) \\
\quad-B_{1} P_{C}\left(p-\mu_{2} B_{2} p\right) \|^{2}
\end{gathered}
$$




$$
\begin{aligned}
\leq & \left\|\left(x_{n}-\mu_{2} B_{2} x_{n}\right)-\left(p-\mu_{2} B_{2} p\right)\right\|^{2} \\
& -\mu_{1}\left(2 \beta_{1}-\mu_{1}\right)\left\|B_{1} \widetilde{x}_{n}-B_{1} q\right\|^{2} \\
= & \left\|\left(x_{n}-p\right)-\mu_{2}\left(B_{2} x_{n}-B_{2} p\right)\right\|^{2} \\
& -\mu_{1}\left(2 \beta_{1}-\mu_{1}\right)\left\|B_{1} \widetilde{x}_{n}-B_{1} q\right\|^{2} \\
\leq & \left\|x_{n}-p\right\|^{2}-\mu_{2}\left(2 \beta_{2}-\mu_{2}\right)\left\|B_{2} x_{n}-B_{2} p\right\|^{2} \\
& -\mu_{1}\left(2 \beta_{1}-\mu_{1}\right)\left\|B_{1} \widetilde{x}_{n}-B_{1} q\right\|^{2} \\
\leq & \left\|x_{n}-p\right\|^{2} .
\end{aligned}
$$

Furthermore, by Proposition 8(ii), we have

$$
\begin{aligned}
& \left\|\bar{u}_{n}-p\right\|^{2} \leq\left\|u_{n}-\lambda_{n} \nabla f_{\alpha_{n}}\left(\widetilde{u}_{n}\right)-p\right\|^{2} \\
& -\left\|u_{n}-\lambda_{n} \nabla f_{\alpha_{n}}\left(\tilde{u}_{n}\right)-\bar{u}_{n}\right\|^{2} \\
& =\left\|u_{n}-p\right\|^{2}-\left\|u_{n}-\bar{u}_{n}\right\|^{2} \\
& +2 \lambda_{n}\left\langle\nabla f_{\alpha_{n}}\left(\widetilde{u}_{n}\right), p-\bar{u}_{n}\right\rangle \\
& =\left\|u_{n}-p\right\|^{2}-\left\|u_{n}-\bar{u}_{n}\right\|^{2} \\
& +2 \lambda_{n}\left(\left\langle\nabla f_{\alpha_{n}}\left(\widetilde{u}_{n}\right)-\nabla f_{\alpha_{n}}(p), p-\widetilde{u}_{n}\right\rangle\right. \\
& +\left\langle\nabla f_{\alpha_{n}}(p), p-\widetilde{u}_{n}\right\rangle \\
& \left.+\left\langle\nabla f_{\alpha_{n}}\left(\widetilde{u}_{n}\right), \tilde{u}_{n}-\bar{u}_{n}\right\rangle\right) \\
& \leq\left\|u_{n}-p\right\|^{2}-\left\|u_{n}-\bar{u}_{n}\right\|^{2} \\
& +2 \lambda_{n}\left(\left\langle\nabla f_{\alpha_{n}}(p), p-\tilde{u}_{n}\right\rangle\right. \\
& \left.+\left\langle\nabla f_{\alpha_{n}}\left(\widetilde{u}_{n}\right), \widetilde{u}_{n}-\bar{u}_{n}\right\rangle\right) \\
& =\left\|u_{n}-p\right\|^{2}-\left\|u_{n}-\bar{u}_{n}\right\|^{2} \\
& +2 \lambda_{n}\left[\left\langle\left(\alpha_{n} I+\nabla f\right) p, p-\widetilde{u}_{n}\right\rangle\right. \\
& \left.+\left\langle\nabla f_{\alpha_{n}}\left(\tilde{u}_{n}\right), \tilde{u}_{n}-\bar{u}_{n}\right\rangle\right] \\
& \leq\left\|u_{n}-p\right\|^{2}-\left\|u_{n}-\bar{u}_{n}\right\|^{2} \\
& +2 \lambda_{n}\left[\alpha_{n}\left\langle p, p-\widetilde{u}_{n}\right\rangle+\left\langle\nabla f_{\alpha_{n}}\left(\widetilde{u}_{n}\right), \widetilde{u}_{n}-\bar{u}_{n}\right\rangle\right] \\
& =\left\|u_{n}-p\right\|^{2}-\left\|u_{n}-\widetilde{u}_{n}\right\|^{2} \\
& -2\left\langle u_{n}-\tilde{u}_{n}, \tilde{u}_{n}-\bar{u}_{n}\right\rangle-\left\|\tilde{u}_{n}-\bar{u}_{n}\right\|^{2} \\
& +2 \lambda_{n}\left[\alpha_{n}\left\langle p, p-\widetilde{u}_{n}\right\rangle+\left\langle\nabla f_{\alpha_{n}}\left(\widetilde{u}_{n}\right), \widetilde{u}_{n}-\bar{u}_{n}\right\rangle\right] \\
& =\left\|u_{n}-p\right\|^{2}-\left\|u_{n}-\widetilde{u}_{n}\right\|^{2}-\left\|\widetilde{u}_{n}-\bar{u}_{n}\right\|^{2}
\end{aligned}
$$$$
+2\left\langle u_{n}-\lambda_{n} \nabla f_{\alpha_{n}}\left(\widetilde{u}_{n}\right)-\widetilde{u}_{n}, \bar{u}_{n}-\widetilde{u}_{n}\right\rangle
$$$$
+2 \lambda_{n} \alpha_{n}\left\langle p, p-\widetilde{u}_{n}\right\rangle \text {. }
$$

Further, by Proposition 8(i), we have

$$
\begin{aligned}
\left\langle u_{n}\right. & \left.-\lambda_{n} \nabla f_{\alpha_{n}}\left(\widetilde{u}_{n}\right)-\widetilde{u}_{n}, \bar{u}_{n}-\widetilde{u}_{n}\right\rangle \\
= & \left\langle u_{n}-\lambda_{n} \nabla f_{\alpha_{n}}\left(u_{n}\right)-\widetilde{u}_{n}, \bar{u}_{n}-\widetilde{u}_{n}\right\rangle \\
& +\left\langle\lambda_{n} \nabla f_{\alpha_{n}}\left(u_{n}\right)-\lambda_{n} \nabla f_{\alpha_{n}}\left(\widetilde{u}_{n}\right), \bar{u}_{n}-\widetilde{u}_{n}\right\rangle \\
\leq & \left\langle\lambda_{n} \nabla f_{\alpha_{n}}\left(u_{n}\right)-\lambda_{n} \nabla f_{\alpha_{n}}\left(\widetilde{u}_{n}\right), \bar{u}_{n}-\widetilde{u}_{n}\right\rangle \\
\leq & \lambda_{n}\left\|\nabla f_{\alpha_{n}}\left(u_{n}\right)-\nabla f_{\alpha_{n}}\left(\widetilde{u}_{n}\right)\right\|\left\|\bar{u}_{n}-\widetilde{u}_{n}\right\| \\
\leq & \lambda_{n}\left(\alpha_{n}+\|A\|^{2}\right)\left\|u_{n}-\widetilde{u}_{n}\right\|\left\|\bar{u}_{n}-\widetilde{u}_{n}\right\| .
\end{aligned}
$$

So, from (45) we obtain

$$
\begin{aligned}
\| \bar{u}_{n}- & p \|^{2} \\
\leq & \left\|u_{n}-p\right\|^{2}-\left\|u_{n}-\widetilde{u}_{n}\right\|^{2}-\left\|\widetilde{u}_{n}-\bar{u}_{n}\right\|^{2} \\
& +2\left\langle u_{n}-\lambda_{n} \nabla f_{\alpha_{n}}\left(\widetilde{u}_{n}\right)-\widetilde{u}_{n}, \bar{u}_{n}-\widetilde{u}_{n}\right\rangle \\
& +2 \lambda_{n} \alpha_{n}\left\langle p, p-\widetilde{u}_{n}\right\rangle \\
\leq & \left\|u_{n}-p\right\|^{2}-\left\|u_{n}-\widetilde{u}_{n}\right\|^{2} \\
& -\left\|\widetilde{u}_{n}-\bar{u}_{n}\right\|^{2}+2 \lambda_{n}\left(\alpha_{n}+\|A\|^{2}\right) \\
& \times\left\|u_{n}-\widetilde{u}_{n}\right\|\left\|\bar{u}_{n}-\widetilde{u}_{n}\right\| \\
& +2 \lambda_{n} \alpha_{n}\|p\|\left\|\widetilde{u}_{n}-p\right\| \\
\leq & \left\|u_{n}-p\right\|^{2}-\left\|u_{n}-\widetilde{u}_{n}\right\|^{2} \\
& -\left\|\widetilde{u}_{n}-\bar{u}_{n}\right\|^{2} \\
& +\lambda_{n}^{2}\left(\alpha_{n}+\|A\|^{2}\right)^{2}\left\|u_{n}-\widetilde{u}_{n}\right\|^{2} \\
& +\left\|\widetilde{u}_{n}-\bar{u}_{n}\right\|^{2}+2 \lambda_{n} \alpha_{n}\|p\|\left\|\widetilde{u}_{n}-p\right\| \\
= & \left\|u_{n}-p\right\|^{2}+2 \lambda_{n} \alpha_{n}\|p\|\left\|\widetilde{u}_{n}-p\right\| \\
& +\left(\lambda_{n}^{2}\left(\alpha_{n}+\|A\|^{2}\right)^{2}-1\right)\left\|u_{n}-\widetilde{u}_{n}\right\|^{2} \\
\leq & \left\|u_{n}-p\right\|^{2}+2 \lambda_{n} \alpha_{n}\|p\|\left\|\widetilde{u}_{n}-p\right\| \\
\leq & \left\|u_{n}-p\right\|^{2} \\
& +2 \lambda_{n} \alpha_{n}\|p\|\left[\left\|u_{n}-p\right\|+\lambda_{n} \alpha_{n}\|p\|\right] \\
\leq & \left\|u_{n}-p\right\|^{2}
\end{aligned}
$$




$$
\begin{aligned}
& \quad+4 \lambda_{n} \alpha_{n}\|p\|\left\|u_{n}-p\right\|+4 \lambda_{n}^{2} \alpha_{n}^{2}\|p\|^{2} \\
& =\left(\left\|u_{n}-p\right\|+2 \lambda_{n} \alpha_{n}\|p\|\right)^{2} .
\end{aligned}
$$

Hence it follows from (48) and (51) that

$$
\begin{aligned}
\left\|y_{n}-p\right\| & \\
= & \left\|\sigma_{n}\left(Q x_{n}-p\right)+\left(1-\sigma_{n}\right)\left(\bar{u}_{n}-p\right)\right\| \\
\leq & \sigma_{n}\left\|Q x_{n}-p\right\|+\left(1-\sigma_{n}\right)\left\|\bar{u}_{n}-p\right\| \\
\leq & \sigma_{n}\left(\left\|Q x_{n}-Q p\right\|+\|Q p-p\|\right) \\
& +\left(1-\sigma_{n}\right)\left(\left\|u_{n}-p\right\|+2 \lambda_{n} \alpha_{n}\|p\|\right) \\
\leq & \sigma_{n}\left(\rho\left\|x_{n}-p\right\|+\|Q p-p\|\right) \\
& +\left(1-\sigma_{n}\right)\left(\left\|x_{n}-p\right\|+2 \lambda_{n} \alpha_{n}\|p\|\right) \\
\leq & \left(1-(1-\rho) \sigma_{n}\right)\left\|x_{n}-p\right\| \\
& +\sigma_{n}\|Q p-p\|+2 \lambda_{n} \alpha_{n}\|p\| \\
= & \left(1-(1-\rho) \sigma_{n}\right)\left\|x_{n}-p\right\| \\
& +(1-\rho) \sigma_{n} \frac{\|Q p-p\|}{1-\rho}+2 \lambda_{n} \alpha_{n}\|p\| \\
\leq & \max \left\{\left\|x_{n}-p\right\|, \frac{\|Q p-p\|}{1-\rho}\right\}+2 \lambda_{n} \alpha_{n}\|p\| .
\end{aligned}
$$

Since $\left(\gamma_{n}+\delta_{n}\right) k \leq \gamma_{n}$ for all $n \geq 0$, utilizing Lemma 17 we obtain from (52)

$$
\begin{aligned}
\left\|x_{n+1}-p\right\| & \\
= & \left\|\beta_{n}\left(x_{n}-p\right)+\gamma_{n}\left(y_{n}-p\right)+\delta_{n}\left(S y_{n}-p\right)\right\| \\
\leq & \beta_{n}\left\|x_{n}-p\right\|+\left\|\gamma_{n}\left(y_{n}-p\right)+\delta_{n}\left(S y_{n}-p\right)\right\| \\
\leq & \beta_{n}\left\|x_{n}-p\right\|+\left(\gamma_{n}+\delta_{n}\right)\left\|y_{n}-p\right\| \\
\leq & \beta_{n}\left\|x_{n}-p\right\| \\
& +\left(\gamma_{n}+\delta_{n}\right)\left[\max \left\{\left\|x_{n}-p\right\|, \frac{\|Q p-p\|}{1-\rho}\right\}+2 \lambda_{n} \alpha_{n}\|p\|\right] \\
\leq & \beta_{n}\left\|x_{n}-p\right\| \\
& +\left(\gamma_{n}+\delta_{n}\right) \max \left\{\left\|x_{n}-p\right\|, \frac{\|Q p-p\|}{1-\rho}\right\}+2 \lambda_{n} \alpha_{n}\|p\| \\
\leq & \max \left\{\left\|x_{n}-p\right\|, \frac{\|Q p-p\|}{1-\rho}\right\}+2 b\|p\| \alpha_{n} .
\end{aligned}
$$

Now, we claim that

$$
\begin{aligned}
\left\|x_{n+1}-p\right\| \leq & \max \left\{\left\|x_{0}-p\right\|, \frac{\|Q p-p\|}{1-\rho}\right\} \\
& +2 b\|p\| \sum_{j=0}^{n} \alpha_{j} .
\end{aligned}
$$

As a matter of fact, if $n=0$, then it is clear that (54) is valid, that is,

$$
\left\|x_{1}-p\right\| \leq \max \left\{\left\|x_{0}-p\right\|, \frac{\|Q p-p\|}{1-\rho}\right\}+2 b\|p\| \sum_{j=0}^{0} \alpha_{j} .
$$

Assume that (54) holds for $n \geq 1$, that is,

$$
\left\|x_{n}-p\right\| \leq \max \left\{\left\|x_{0}-p\right\|, \frac{\|Q p-p\|}{1-\rho}\right\}+2 b\|p\| \sum_{j=0}^{n-1} \alpha_{j} .
$$

Then, we conclude from (53) and (56) that

$$
\begin{aligned}
& \left\|x_{n+1}-p\right\| \\
& \leq \max \left\{\left\|x_{n}-p\right\|, \frac{\|Q p-p\|}{1-\rho}\right\}+2 b\|p\| \alpha_{n} \\
& \leq \max \left\{\max \left\{\left\|x_{0}-p\right\|, \frac{\|Q p-p\|}{1-\rho}\right\}\right. \\
& \left.+2 b\|p\| \sum_{j=0}^{n-1} \alpha_{j}, \frac{\|Q p-p\|}{1-\rho}\right\}+2 b\|p\| \alpha_{n} \\
& \leq \max \left\{\left\|x_{0}-p\right\|, \frac{\|Q p-p\|}{1-\rho}\right\} \\
& +2 b\|p\| \sum_{j=0}^{n-1} \alpha_{j}+2 b\|p\| \alpha_{n} \\
& =\max \left\{\left\|x_{0}-p\right\|, \frac{\|Q p-p\|}{1-\rho}\right\} \\
& \quad+2 b\|p\| \sum_{j=0}^{n} \alpha_{j} .
\end{aligned}
$$

By induction, we conclude that (54) is valid. Hence, $\left\{x_{n}\right\}$ is bounded. Since $P_{C}, \nabla f_{\alpha_{n}}, B_{1}$ and $B_{2}$ are Lipschitz continuous, it is easy to see that $\left\{u_{n}\right\},\left\{\tilde{u}_{n}\right\},\left\{\bar{u}_{n}\right\},\left\{y_{n}\right\}$ and $\left\{\tilde{x}_{n}\right\}$ are bounded, where $\widetilde{x}_{n}=P_{C}\left(x_{n}-\mu_{2} B_{2} x_{n}\right)$ for all $n \geq 0$.

Step 2. $\lim _{n \rightarrow \infty}\left\|x_{n+1}-x_{n}\right\|=0$. 
Indeed, define $x_{n+1}=\beta_{n} x_{n}+\left(1-\beta_{n}\right) w_{n}$ for all $n \geq 0$. It follows that

$$
\begin{aligned}
w_{n+1}-w_{n}= & \frac{x_{n+2}-\beta_{n+1} x_{n+1}}{1-\beta_{n+1}}-\frac{x_{n+1}-\beta_{n} x_{n}}{1-\beta_{n}} \\
= & \frac{\gamma_{n+1} y_{n+1}+\delta_{n+1} S y_{n+1}}{1-\beta_{n+1}}-\frac{\gamma_{n} y_{n}+\delta_{n} S y_{n}}{1-\beta_{n}} \\
= & \frac{\gamma_{n+1}\left(y_{n+1}-y_{n}\right)+\delta_{n+1}\left(S y_{n+1}-S y_{n}\right)}{1-\beta_{n+1}} \\
& +\left(\frac{\gamma_{n+1}}{1-\beta_{n+1}}-\frac{\gamma_{n}}{1-\beta_{n}}\right) y_{n} \\
& +\left(\frac{\delta_{n+1}}{1-\beta_{n+1}}-\frac{\delta_{n}}{1-\beta_{n}}\right) S y_{n} .
\end{aligned}
$$

Since $\left(\gamma_{n}+\delta_{n}\right) k \leq \gamma_{n}$ for all $n \geq 0$, utilizing Lemma 17 we have

$$
\begin{gathered}
\left\|\gamma_{n+1}\left(y_{n+1}-y_{n}\right)+\delta_{n+1}\left(S y_{n+1}-S y_{n}\right)\right\| \\
\leq\left(\gamma_{n+1}+\delta_{n+1}\right)\left\|y_{n+1}-y_{n}\right\| .
\end{gathered}
$$

Next, we estimate $\left\|y_{n+1}-y_{n}\right\|$. Observe that

$$
\begin{aligned}
\| \tilde{u}_{n+1} & -\tilde{u}_{n} \| \\
= & \| P_{C}\left(u_{n+1}-\lambda_{n+1} \nabla f_{\alpha_{n+1}}\left(u_{n+1}\right)\right) \\
& -P_{C}\left(u_{n}-\lambda_{n} \nabla f_{\alpha_{n}}\left(u_{n}\right)\right) \| \\
\leq & \| P_{C}\left(I-\lambda_{n+1} \nabla f_{\alpha_{n+1}}\right) u_{n+1} \\
& -P_{C}\left(I-\lambda_{n+1} \nabla f_{\alpha_{n+1}}\right) u_{n} \| \\
& +\left\|P_{C}\left(I-\lambda_{n+1} \nabla f_{\alpha_{n+1}}\right) u_{n}-P_{C}\left(I-\lambda_{n} \nabla f_{\alpha_{n}}\right) u_{n}\right\| \\
\leq & \left\|u_{n+1}-u_{n}\right\| \\
& +\left\|\left(I-\lambda_{n+1} \nabla f_{\alpha_{n+1}}\right) u_{n}-\left(I-\lambda_{n} \nabla f_{\alpha_{n}}\right) u_{n}\right\| \\
= & \left\|u_{n+1}-u_{n}\right\| \\
& +\left\|\lambda_{n+1}\left(\alpha_{n+1} I+\nabla f\right) u_{n}-\lambda_{n}\left(\alpha_{n} I+\nabla f\right) u_{n}\right\| \\
\leq & \left\|u_{n+1}-u_{n}\right\| \\
& +\left|\lambda_{n+1}-\lambda_{n}\right|\left\|\nabla f\left(u_{n}\right)\right\|+\left|\lambda_{n+1} \alpha_{n+1}-\lambda_{n} \alpha_{n}\right|\left\|u_{n}\right\| \\
= & \left.\left.\quad P_{C}\left[P_{C}\left(x_{n}-\mu_{2} B_{2} x_{n}\right)-\mu_{1} B_{1} P_{C}\left(x_{n}-\mu_{2} B_{2} x_{n}\right)\right] \|^{2} B_{2} x_{n}\right)-\mu_{1} B_{1} P_{C}\left(x_{n}-\mu_{2} B_{2} x_{n}\right)\right] \|^{2} \\
\| u_{n+1}- & u_{n} \|^{2} \\
= & P_{C}\left[P_{C}\left(x_{n+1}-\mu_{2} B_{2} x_{n+1}\right)-\mu_{1} B_{1} P_{C}\left(x_{n+1}-\mu_{2} B_{2} x_{n+1}\right)\right]
\end{aligned}
$$$$
=\|\left[P_{C}\left(x_{n+1}-\mu_{2} B_{2} x_{n+1}\right)-P_{C}\left(x_{n}-\mu_{2} B_{2} x_{n}\right)\right]
$$$$
-\mu_{1}\left[B_{1} P_{C}\left(x_{n+1}-\mu_{2} B_{2} x_{n+1}\right)-B_{1} P_{C}\left(x_{n}-\mu_{2} B_{2} x_{n}\right)\right] \|^{2}
$$$$
\leq\left\|P_{C}\left(x_{n+1}-\mu_{2} B_{2} x_{n+1}\right)-P_{C}\left(x_{n}-\mu_{2} B_{2} x_{n}\right)\right\|^{2}
$$$$
-\mu_{1}\left(2 \beta_{1}-\mu_{1}\right)
$$$$
\times\left\|B_{1} P_{C}\left(x_{n+1}-\mu_{2} B_{2} x_{n+1}\right)-B_{1} P_{C}\left(x_{n}-\mu_{2} B_{2} x_{n}\right)\right\|^{2}
$$$$
\leq\left\|P_{C}\left(x_{n+1}-\mu_{2} B_{2} x_{n+1}\right)-P_{C}\left(x_{n}-\mu_{2} B_{2} x_{n}\right)\right\|^{2}
$$$$
\leq\left\|\left(x_{n+1}-\mu_{2} B_{2} x_{n+1}\right)-\left(x_{n}-\mu_{2} B_{2} x_{n}\right)\right\|^{2}
$$$$
=\left\|\left(x_{n+1}-x_{n}\right)-\mu_{2}\left(B_{2} x_{n+1}-B_{2} x_{n}\right)\right\|^{2}
$$$$
\leq\left\|x_{n+1}-x_{n}\right\|^{2}-\mu_{2}\left(2 \beta_{2}-\mu_{2}\right)\left\|B_{2} x_{n+1}-B_{2} x_{n}\right\|^{2}
$$$$
\leq\left\|x_{n+1}-x_{n}\right\|^{2} \text {, }
$$

and hence

$$
\begin{aligned}
& \left\|\bar{u}_{n+1}-\bar{u}_{n}\right\| \\
& =\| P_{C}\left(u_{n+1}-\lambda_{n+1} \nabla f_{\alpha_{n+1}}\left(\tilde{u}_{n+1}\right)\right) \\
& -P_{C}\left(u_{n}-\lambda_{n} \nabla f_{\alpha_{n}}\left(\tilde{u}_{n}\right)\right) \| \\
& \leq\left\|\left(u_{n+1}-\lambda_{n+1} \nabla f_{\alpha_{n+1}}\left(\tilde{u}_{n+1}\right)\right)-\left(u_{n}-\lambda_{n} \nabla f_{\alpha_{n}}\left(\tilde{u}_{n}\right)\right)\right\| \\
& =\|\left(I-\lambda_{n+1} \nabla f_{\alpha_{n+1}}\right) u_{n+1}-\left(I-\lambda_{n+1} \nabla f_{\alpha_{n+1}}\right) u_{n} \\
& +\lambda_{n+1}\left(\nabla f_{\alpha_{n+1}}\left(u_{n+1}\right)-\nabla f_{\alpha_{n+1}}\left(u_{n}\right)\right) \\
& -\lambda_{n+1} \nabla f_{\alpha_{n+1}}\left(\tilde{u}_{n+1}\right)+\lambda_{n} \nabla f_{\alpha_{n}}\left(\tilde{u}_{n}\right) \| \\
& \leq\left\|\left(I-\lambda_{n+1} \nabla f_{\alpha_{n+1}}\right) u_{n+1}-\left(I-\lambda_{n+1} \nabla f_{\alpha_{n+1}}\right) u_{n}\right\| \\
& +\lambda_{n+1}\left\|\nabla f_{\alpha_{n+1}}\left(u_{n+1}\right)-\nabla f_{\alpha_{n+1}}\left(u_{n}\right)\right\| \\
& +\left\|\lambda_{n+1} \nabla f_{\alpha_{n+1}}\left(\tilde{u}_{n+1}\right)-\lambda_{n} \nabla f_{\alpha_{n}}\left(\tilde{u}_{n}\right)\right\| \\
& \leq\left\|u_{n+1}-u_{n}\right\|+\lambda_{n+1}\left(\alpha_{n+1}+\|A\|^{2}\right)\left\|u_{n+1}-u_{n}\right\| \\
& +\left\|\lambda_{n+1}\left(\alpha_{n+1} I+\nabla f\right)\left(\widetilde{u}_{n+1}\right)-\lambda_{n}\left(\alpha_{n} I+\nabla f\right)\left(\widetilde{u}_{n}\right)\right\| \\
& \leq\left\|u_{n+1}-u_{n}\right\|+\lambda_{n+1}\left(\alpha_{n+1}+\|A\|^{2}\right)\left\|u_{n+1}-u_{n}\right\| \\
& +\left\|\lambda_{n+1} \alpha_{n+1} \tilde{u}_{n+1}-\lambda_{n} \alpha_{n} \tilde{u}_{n}\right\| \\
& +\left\|\lambda_{n+1} \nabla f\left(\tilde{u}_{n+1}\right)-\lambda_{n} \nabla f\left(\tilde{u}_{n}\right)\right\| \\
& \leq\left\|u_{n+1}-u_{n}\right\|+\lambda_{n+1}\left(\alpha_{n+1}+\|A\|^{2}\right)\left\|u_{n+1}-u_{n}\right\| \\
& +\left\|\lambda_{n+1} \alpha_{n+1} \tilde{u}_{n+1}-\lambda_{n} \alpha_{n} \tilde{u}_{n}\right\| \\
& +\lambda_{n+1}\left\|\nabla f\left(\tilde{u}_{n+1}\right)-\nabla f\left(\tilde{u}_{n}\right)\right\|+\left|\lambda_{n+1}-\lambda_{n}\right|\left\|\nabla f\left(\tilde{u}_{n}\right)\right\|
\end{aligned}
$$




$$
\begin{aligned}
\leq & \left\|x_{n+1}-x_{n}\right\|+\lambda_{n+1}\left(\alpha_{n+1}+\|A\|^{2}\right)\left\|u_{n+1}-u_{n}\right\| \\
& +\left\|\lambda_{n+1} \alpha_{n+1} \widetilde{u}_{n+1}-\lambda_{n} \alpha_{n} \widetilde{u}_{n}\right\| \\
& +\left|\lambda_{n+1}-\lambda_{n}\right|\left\|\nabla f\left(\widetilde{u}_{n}\right)\right\|+\lambda_{n+1}\|A\|^{2}\left\|\tilde{u}_{n+1}-\widetilde{u}_{n}\right\| \\
\leq & \left\|x_{n+1}-x_{n}\right\|+\lambda_{n+1}\left(\alpha_{n+1}+\|A\|^{2}\right) \\
& \times\left(\left\|u_{n+1}-u_{n}\right\|+\left\|\widetilde{u}_{n+1}-\widetilde{u}_{n}\right\|\right) \\
& +\left\|\lambda_{n+1} \alpha_{n+1} \widetilde{u}_{n+1}-\lambda_{n} \alpha_{n} \widetilde{u}_{n}\right\| \\
& +\left|\lambda_{n+1}-\lambda_{n}\right|\left\|\nabla f\left(\widetilde{u}_{n}\right)\right\| .
\end{aligned}
$$

Combining (60) with (61), we get

$$
\begin{aligned}
\left\|\widetilde{u}_{n+1}-\widetilde{u}_{n}\right\| \leq & \left\|u_{n+1}-u_{n}\right\|+\left|\lambda_{n+1}-\lambda_{n}\right|\left\|\nabla f\left(u_{n}\right)\right\| \\
& +\left|\lambda_{n+1} \alpha_{n+1}-\lambda_{n} \alpha_{n}\right|\left\|u_{n}\right\| \\
\leq & \left\|x_{n+1}-x_{n}\right\|+\left|\lambda_{n+1}-\lambda_{n}\right|\left\|\nabla f\left(u_{n}\right)\right\| \\
& +\left|\lambda_{n+1} \alpha_{n+1}-\lambda_{n} \alpha_{n}\right|\left\|u_{n}\right\| .
\end{aligned}
$$

This together with (62) implies that

$$
\begin{aligned}
\| y_{n+1} & -y_{n} \| \\
= & \| \bar{u}_{n+1}+\sigma_{n+1}\left(Q x_{n+1}-\bar{u}_{n+1}\right) \\
& \quad-\bar{u}_{n}-\sigma_{n}\left(Q x_{n}-\bar{u}_{n}\right) \| \\
\leq & \left\|\bar{u}_{n+1}-\bar{u}_{n}\right\|+\sigma_{n+1}\left\|Q x_{n+1}-\bar{u}_{n+1}\right\| \\
& +\sigma_{n}\left\|Q x_{n}-\bar{u}_{n}\right\| \\
\leq & \left\|x_{n+1}-x_{n}\right\| \\
& +\lambda_{n+1}\left(\alpha_{n+1}+\|A\|^{2}\right)\left(\left\|u_{n+1}-u_{n}\right\|+\left\|\widetilde{u}_{n+1}-\widetilde{u}_{n}\right\|\right) \\
& +\left\|\lambda_{n+1} \alpha_{n+1} \widetilde{u}_{n+1}-\lambda_{n} \alpha_{n} \widetilde{u}_{n}\right\|+\left|\lambda_{n+1}-\lambda_{n}\right|\left\|\nabla f\left(\widetilde{u}_{n}\right)\right\| \\
& +\sigma_{n+1}\left\|Q x_{n+1}-\bar{u}_{n+1}\right\|+\sigma_{n}\left\|Q x_{n}-\bar{u}_{n}\right\| .
\end{aligned}
$$

Hence it follows from (58), (59), and (64) that

$$
\begin{aligned}
& \left\|w_{n+1}-w_{n}\right\| \\
& \leq \quad \frac{\left\|\gamma_{n+1}\left(y_{n+1}-y_{n}\right)+\delta_{n+1}\left(S y_{n+1}-S y_{n}\right)\right\|}{1-\beta_{n+1}} \\
& \quad+\left|\frac{\gamma_{n+1}}{1-\beta_{n+1}}-\frac{\gamma_{n}}{1-\beta_{n}}\right|\left\|y_{n}\right\|+\left|\frac{\delta_{n+1}}{1-\beta_{n+1}}-\frac{\delta_{n}}{1-\beta_{n}}\right|\left\|S y_{n}\right\| \\
& \leq \quad \frac{\gamma_{n+1}+\delta_{n+1}}{1-\beta_{n+1}}\left\|y_{n+1}-y_{n}\right\| \\
& \quad+\left|\frac{\gamma_{n+1}}{1-\beta_{n+1}}-\frac{\gamma_{n}}{1-\beta_{n}}\right|\left(\left\|y_{n}\right\|+\left\|S y_{n}\right\|\right)
\end{aligned}
$$

$$
\begin{aligned}
= & \left\|y_{n+1}-y_{n}\right\|+\left|\frac{\gamma_{n+1}}{1-\beta_{n+1}}-\frac{\gamma_{n}}{1-\beta_{n}}\right|\left(\left\|y_{n}\right\|+\left\|S y_{n}\right\|\right) \\
\leq & \left\|x_{n+1}-x_{n}\right\| \\
& +\lambda_{n+1}\left(\alpha_{n+1}+\|A\|^{2}\right)\left(\left\|u_{n+1}-u_{n}\right\|+\left\|\tilde{u}_{n+1}-\tilde{u}_{n}\right\|\right) \\
& +\left\|\lambda_{n+1} \alpha_{n+1} \tilde{u}_{n+1}-\lambda_{n} \alpha_{n} \tilde{u}_{n}\right\|+\left|\lambda_{n+1}-\lambda_{n}\right|\left\|\nabla f\left(\tilde{u}_{n}\right)\right\| \\
& +\sigma_{n+1}\left\|Q x_{n+1}-\bar{u}_{n+1}\right\|+\sigma_{n}\left\|Q x_{n}-\bar{u}_{n}\right\| \\
& +\left|\frac{\gamma_{n+1}}{1-\beta_{n+1}}-\frac{\gamma_{n}}{1-\beta_{n}}\right|\left(\left\|y_{n}\right\|+\left\|S y_{n}\right\|\right) .
\end{aligned}
$$

From (60), we deduce from condition (vi) that $\lim _{n \rightarrow \infty}$ $\left\|\widetilde{u}_{n+1}-\widetilde{u}_{n}\right\|=0$. Since $\left\{x_{n}\right\},\left\{u_{n}\right\},\left\{\tilde{u}_{n}\right\},\left\{y_{n}\right\}$ and $\left\{\bar{u}_{n}\right\}$ are bounded, it follows from conditions (i), (iii), (v), and (vi) that

$$
\begin{aligned}
\limsup _{n \rightarrow \infty}\left(\left\|w_{n+1}-w_{n}\right\|-\left\|x_{n+1}-x_{n}\right\|\right) \\
\leq \limsup _{n \rightarrow \infty}\left\{\lambda_{n+1}\left(\alpha_{n+1}+\|A\|^{2}\right)\right. \\
\times \\
+\left\|\lambda_{n+1} \alpha_{n+1} \widetilde{u}_{n+1}-\lambda_{n} \alpha_{n} \tilde{u}_{n}\right\| \\
+\left|\lambda_{n+1}-\lambda_{n}\right|\left\|\nabla f\left(\tilde{u}_{n}\right)\right\| \\
+\sigma_{n+1}\left\|Q x_{n+1}-\bar{u}_{n+1}\right\|+\sigma_{n}\left\|Q x_{n}-\bar{u}_{n}\right\| \\
\left.+\left|\frac{\gamma_{n+1}}{1-\beta_{n+1}}-\frac{\gamma_{n}}{1-\beta_{n}}\right|\left(\left\|y_{n}\right\|+\left\|S y_{n}\right\|\right)\right\}=0
\end{aligned}
$$

Hence by Lemma 14 we get $\lim _{n \rightarrow \infty}\left\|w_{n}-x_{n}\right\|=0$. Thus,

$$
\lim _{n \rightarrow \infty}\left\|x_{n+1}-x_{n}\right\|=\lim _{n \rightarrow \infty}\left(1-\beta_{n}\right)\left\|w_{n}-x_{n}\right\|=0 .
$$

Step 3. $\lim _{n \rightarrow \infty}\left\|B_{2} x_{n}-B_{2} p\right\|=0, \lim _{n \rightarrow \infty}\left\|B_{1} \tilde{x}_{n}-B_{1} q\right\|=0$, and $\lim _{n \rightarrow \infty}\left\|u_{n}-\widetilde{u}_{n}\right\|=0$, where $q=P_{C}\left(p-\mu_{2} B_{2} p\right)$.

Indeed, utilizing Lemma 17 and the convexity of $\|\cdot\|^{2}$, we obtain from (17), (48), and (51) that

$$
\begin{aligned}
& \left\|x_{n+1}-p\right\|^{2} \\
& =\left\|\beta_{n}\left(x_{n}-p\right)+\gamma_{n}\left(y_{n}-p\right)+\delta_{n}\left(S y_{n}-p\right)\right\|^{2} \\
& \leq \beta_{n}\left\|x_{n}-p\right\|^{2} \\
& \quad+\left(\gamma_{n}+\delta_{n}\right)\left\|\frac{1}{\gamma_{n}+\delta_{n}}\left[\gamma_{n}\left(y_{n}-p\right)+\delta_{n}\left(S y_{n}-p\right)\right]\right\|^{2} \\
& \quad \beta_{n}\left\|x_{n}-p\right\|^{2}+\left(\gamma_{n}+\delta_{n}\right)\left\|y_{n}-p\right\|^{2}
\end{aligned}
$$




$$
\begin{aligned}
\leq & \beta_{n}\left\|x_{n}-p\right\|^{2} \\
& +\left(\gamma_{n}+\delta_{n}\right)\left[\sigma_{n}\left\|Q x_{n}-p\right\|^{2}+\left(1-\sigma_{n}\right)\left\|\bar{u}_{n}-p\right\|^{2}\right] \\
\leq & \beta_{n}\left\|x_{n}-p\right\|^{2}+\sigma_{n}\left\|Q x_{n}-p\right\|^{2}+\left(\gamma_{n}+\delta_{n}\right)\left\|\bar{u}_{n}-p\right\|^{2} \\
\leq & \beta_{n}\left\|x_{n}-p\right\|^{2}+\sigma_{n}\left\|Q x_{n}-p\right\|^{2} \\
& +\left(\gamma_{n}+\delta_{n}\right)\left[\left\|u_{n}-p\right\|^{2}+2 \lambda_{n} \alpha_{n}\|p\|\left\|\widetilde{u}_{n}-p\right\|\right. \\
& \left.\quad+\left(\lambda_{n}^{2}\left(\alpha_{n}+\|A\|^{2}\right)^{2}-1\right)\left\|u_{n}-\widetilde{u}_{n}\right\|^{2}\right] \\
\leq & \beta_{n}\left\|x_{n}-p\right\|^{2}+\sigma_{n}\left\|Q x_{n}-p\right\|^{2}+\left(\gamma_{n}+\delta_{n}\right) \\
& \times\left[\left\|x_{n}-p\right\|^{2}-\mu_{2}\left(2 \beta_{2}-\mu_{2}\right)\left\|B_{2} x_{n}-B_{2} p\right\|^{2}\right. \\
& -\mu_{1}\left(2 \beta_{1}-\mu_{1}\right)\left\|B_{1} \tilde{x}_{n}-B_{1} q\right\|^{2}+2 \lambda_{n} \alpha_{n}\|p\|\left\|\tilde{u}_{n}-p\right\| \\
& \left.+\left(\lambda_{n}^{2}\left(\alpha_{n}+\|A\|^{2}\right)^{2}-1\right)\left\|u_{n}-\widetilde{u}_{n}\right\|^{2}\right] \\
\leq & \left\|x_{n}-p\right\|^{2}+\sigma_{n}\left\|Q x_{n}-p\right\|^{2}-\left(\gamma_{n}+\delta_{n}\right) \\
& \times\left[\mu_{2}\left(2 \beta_{2}-\mu_{2}\right)\left\|B_{2} x_{n}-B_{2} p\right\|^{2}\right. \\
& +\mu_{1}\left(2 \beta_{1}-\mu_{1}\right)\left\|B_{1} \widetilde{x}_{n}-B_{1} q\right\|^{2} \\
& \left.+\left(1-\lambda_{n}^{2}\left(\alpha_{n}+\|A\|^{2}\right)^{2}\right)\left\|u_{n}-\widetilde{u}_{n}\right\|^{2}\right] \\
& +\alpha_{n}\|p\|\left\|\widetilde{u}_{n}-p\right\| .
\end{aligned}
$$

Therefore,

$$
\begin{aligned}
\left(\gamma_{n}+\delta_{n}\right) & {\left[\mu_{2}\left(2 \beta_{2}-\mu_{2}\right)\left\|B_{2} x_{n}-B_{2} p\right\|^{2}\right.} \\
+ & \mu_{1}\left(2 \beta_{1}-\mu_{1}\right)\left\|B_{1} \tilde{x}_{n}-B_{1} q\right\|^{2} \\
& \left.+\left(1-\lambda_{n}^{2}\left(\alpha_{n}+\|A\|^{2}\right)^{2}\right)\left\|u_{n}-\widetilde{u}_{n}\right\|^{2}\right] \\
\leq & \left\|x_{n}-p\right\|^{2}-\left\|x_{n+1}-p\right\|^{2}+\sigma_{n}\left\|Q x_{n}-p\right\|^{2} \\
+ & 2 \lambda_{n} \alpha_{n}\|p\|\left\|\widetilde{u}_{n}-p\right\| \\
\leq & \left(\left\|x_{n}-p\right\|+\left\|x_{n+1}-p\right\|\right)\left\|x_{n}-x_{n+1}\right\|+\sigma_{n}\left\|Q x_{n}-p\right\|^{2} \\
+ & 2 \lambda_{n} \alpha_{n}\|p\|\left\|\tilde{u}_{n}-p\right\| .
\end{aligned}
$$

Since $\alpha_{n} \rightarrow 0, \sigma_{n} \rightarrow 0,\left\|x_{n}-x_{n+1}\right\| \rightarrow 0, \liminf _{n \rightarrow \infty}\left(\gamma_{n}+\right.$ $\left.\delta_{n}\right)>0$ and $\left\{\lambda_{n}\right\} \subset[a, b]$ for some $a, b \in\left(0,1 /\|A\|^{2}\right)$, it follows that

$$
\begin{gathered}
\lim _{n \rightarrow \infty}\left\|u_{n}-\widetilde{u}_{n}\right\|=0, \quad \lim _{n \rightarrow \infty}\left\|B_{1} \widetilde{x}_{n}-B_{1} q\right\|=0, \\
\lim _{n \rightarrow \infty}\left\|B_{2} x_{n}-B_{2} p\right\|=0 .
\end{gathered}
$$

Step 4. $\lim _{n \rightarrow \infty}\left\|S y_{n}-y_{n}\right\|=0$.
Indeed, observe that

$$
\begin{aligned}
& \left\|\bar{u}_{n}-\widetilde{u}_{n}\right\| \\
& \quad=\left\|P_{C}\left(u_{n}-\lambda_{n} \nabla f_{\alpha_{n}}\left(\widetilde{u}_{n}\right)\right)-P_{C}\left(u_{n}-\lambda_{n} \nabla f_{\alpha_{n}}\left(u_{n}\right)\right)\right\| \\
& \quad \leq\left\|\left(u_{n}-\lambda_{n} \nabla f_{\alpha_{n}}\left(\widetilde{u}_{n}\right)\right)-\left(u_{n}-\lambda_{n} \nabla f_{\alpha_{n}}\left(u_{n}\right)\right)\right\| \\
& =\lambda_{n}\left\|\nabla f_{\alpha_{n}}\left(\widetilde{u}_{n}\right)-\nabla f_{\alpha_{n}}\left(u_{n}\right)\right\| \\
& \quad \leq \lambda_{n}\left(\alpha_{n}+\|A\|^{2}\right)\left\|\widetilde{u}_{n}-u_{n}\right\| .
\end{aligned}
$$

This together with $\left\|\widetilde{u}_{n}-u_{n}\right\| \rightarrow 0$ implies that $\lim _{n \rightarrow \infty} \| \bar{u}_{n}-$ $\tilde{u}_{n} \|=0$ and hence $\lim _{n \rightarrow \infty}\left\|\bar{u}_{n}-u_{n}\right\|=0$. By firm nonexpansiveness of $P_{C}$, we have

$$
\begin{aligned}
\left\|\tilde{x}_{n}-q\right\|^{2} & \\
= & \left\|P_{C}\left(x_{n}-\mu_{2} B_{2} x_{n}\right)-P_{C}\left(p-\mu_{2} B_{2} p\right)\right\|^{2} \\
\leq & \left\langle\left(x_{n}-\mu_{2} B_{2} x_{n}\right)-\left(p-\mu_{2} B_{2} p\right), \tilde{x}_{n}-q\right\rangle \\
= & \frac{1}{2}\left[\left\|x_{n}-p-\mu_{2}\left(B_{2} x_{n}-B_{2} p\right)\right\|^{2}+\left\|\tilde{x}_{n}-q\right\|^{2}\right. \\
& \left.-\left\|\left(x_{n}-p\right)-\mu_{2}\left(B_{2} x_{n}-B_{2} p\right)-\left(\tilde{x}_{n}-q\right)\right\|^{2}\right] \\
\leq & \frac{1}{2}\left[\left\|x_{n}-p\right\|^{2}+\left\|\tilde{x}_{n}-q\right\|^{2}\right. \\
& \left.\quad-\left\|\left(x_{n}-\tilde{x}_{n}\right)-\mu_{2}\left(B_{2} x_{n}-B_{2} p\right)-(p-q)\right\|^{2}\right] \\
= & \frac{1}{2}\left[\left\|x_{n}-p\right\|^{2}+\left\|\tilde{x}_{n}-q\right\|^{2}-\left\|x_{n}-\tilde{x}_{n}-(p-q)\right\|^{2}\right. \\
& +2 \mu_{2}\left\langle x_{n}-\tilde{x}_{n}-(p-q), B_{2} x_{n}-B_{2} p\right\rangle \\
& \left.\quad-\mu_{2}^{2}\left\|B_{2} x_{n}-B_{2} p\right\|^{2}\right] \\
\leq & \frac{1}{2}\left[\left\|x_{n}-p\right\|^{2}+\left\|\tilde{x}_{n}-q\right\|^{2}\right. \\
\quad & \quad\left\|x_{n}-\tilde{x}_{n}-(p-q)\right\|^{2} \\
& \left.+2 \mu_{2}\left\|x_{n}-\tilde{x}_{n}-(p-q)\right\|\left\|B_{2} x_{n}-B_{2} p\right\|\right],
\end{aligned}
$$

that is,

$$
\begin{aligned}
\left\|\tilde{x}_{n}-q\right\|^{2} \leq & \left\|x_{n}-p\right\|^{2}-\left\|x_{n}-\tilde{x}_{n}-(p-q)\right\|^{2} \\
& +2 \mu_{2}\left\|x_{n}-\tilde{x}_{n}-(p-q)\right\|\left\|B_{2} x_{n}-B_{2} p\right\| .
\end{aligned}
$$


Moreover, using the argument technique similar to the above one, we derive

$$
\begin{aligned}
& \left\|u_{n}-p\right\|^{2} \\
& =\left\|P_{C}\left(\tilde{x}_{n}-\mu_{1} B_{1} \tilde{x}_{n}\right)-P_{C}\left(q-\mu_{1} B_{1} q\right)\right\|^{2} \\
& \leq\left\langle\left(\tilde{x}_{n}-\mu_{1} B_{1} \tilde{x}_{n}\right)-\left(q-\mu_{1} B_{1} q\right), u_{n}-p\right\rangle \\
& =\frac{1}{2}\left[\left\|\tilde{x}_{n}-q-\mu_{1}\left(B_{1} \tilde{x}_{n}-B_{1} q\right)\right\|^{2}+\left\|u_{n}-p\right\|^{2}\right. \\
& \left.\quad-\left\|\left(\tilde{x}_{n}-q\right)-\mu_{1}\left(B_{1} \tilde{x}_{n}-B_{1} q\right)-\left(u_{n}-p\right)\right\|^{2}\right] \\
& \leq \frac{1}{2}\left[\left\|\tilde{x}_{n}-q\right\|^{2}+\left\|u_{n}-p\right\|^{2}\right. \\
& \left.\quad-\left\|\left(\tilde{x}_{n}-u_{n}\right)-\mu_{1}\left(B_{1} \tilde{x}_{n}-B_{1} q\right)+(p-q)\right\|^{2}\right] \\
& =\frac{1}{2}\left[\left\|\tilde{x}_{n}-q\right\|^{2}+\left\|u_{n}-p\right\|^{2}-\left\|\tilde{x}_{n}-u_{n}+(p-q)\right\|^{2}\right. \\
& \quad+2 \mu_{1}\left\langle\tilde{x}_{n}-u_{n}+(p-q), B_{1} \tilde{x}_{n}-B_{1} q\right\rangle \\
& \left.\quad-\mu_{1}^{2}\left\|B_{1} \tilde{x}_{n}-B_{1} q\right\|^{2}\right] \\
& \leq \frac{1}{2}\left[\left\|\tilde{x}_{n}-q\right\|^{2}+\left\|u_{n}-p\right\|^{2}-\left\|\tilde{x}_{n}-u_{n}+(p-q)\right\|^{2}\right. \\
& \left.\quad+2 \tilde{x}_{n}-u_{n}+(p-q)\|\| B_{1} \tilde{x}_{n}-B_{1} q \|\right]
\end{aligned}
$$

that is,

$$
\begin{aligned}
\left\|u_{n}-p\right\|^{2} \leq & \left\|\tilde{x}_{n}-q\right\|^{2}-\left\|\widetilde{x}_{n}-u_{n}+(p-q)\right\|^{2} \\
& +2 \mu_{1}\left\|\widetilde{x}_{n}-u_{n}+(p-q)\right\|\left\|B_{1} \widetilde{x}_{n}-B_{1} q\right\| .
\end{aligned}
$$

Utilizing (46), (73), and (75), we have

$$
\begin{aligned}
&\left\|\bar{u}_{n}-p\right\|^{2} \\
&=\left\|\widetilde{u}_{n}-p+\bar{u}_{n}-\widetilde{u}_{n}\right\|^{2} \\
& \leq\left\|\widetilde{u}_{n}-p\right\|^{2}+2\left\langle\bar{u}_{n}-\widetilde{u}_{n}, \bar{u}_{n}-p\right\rangle \\
& \leq\left\|\widetilde{u}_{n}-p\right\|^{2}+2\left\|\bar{u}_{n}-\widetilde{u}_{n}\right\|\left\|\bar{u}_{n}-p\right\| \\
& \leq\left\|u_{n}-p\right\|^{2}+2 \lambda_{n} \alpha_{n}\|p\|\left\|\widetilde{u}_{n}-p\right\| \\
&+2\left\|\bar{u}_{n}-\widetilde{u}_{n}\right\|\left\|\bar{u}_{n}-p\right\| \\
& \leq\left\|\widetilde{x}_{n}-q\right\|^{2}-\left\|\tilde{x}_{n}-u_{n}+(p-q)\right\|^{2} \\
&+2 \mu_{1}\left\|\widetilde{x}_{n}-u_{n}+(p-q)\right\|\left\|B_{1} \widetilde{x}_{n}-B_{1} q\right\| \\
&+2 \lambda_{n} \alpha_{n}\|p\|\left\|\widetilde{u}_{n}-p\right\|+2\left\|\bar{u}_{n}-\widetilde{u}_{n}\right\|\left\|\bar{u}_{n}-p\right\|
\end{aligned}
$$

$$
\begin{aligned}
\leq & \left\|x_{n}-p\right\|^{2}-\left\|x_{n}-\tilde{x}_{n}-(p-q)\right\|^{2} \\
& +2 \mu_{2}\left\|x_{n}-\tilde{x}_{n}-(p-q)\right\|\left\|B_{2} x_{n}-B_{2} p\right\| \\
& -\left\|\tilde{x}_{n}-u_{n}+(p-q)\right\|^{2} \\
& +2 \mu_{1}\left\|\tilde{x}_{n}-u_{n}+(p-q)\right\|\left\|B_{1} \tilde{x}_{n}-B_{1} q\right\| \\
& +2 \lambda_{n} \alpha_{n}\|p\|\left\|\tilde{u}_{n}-p\right\|+2\left\|\bar{u}_{n}-\tilde{u}_{n}\right\|\left\|\bar{u}_{n}-p\right\| .
\end{aligned}
$$

Thus from (17) and (76) it follows that

$$
\begin{aligned}
& \left\|x_{n+1}-p\right\|^{2} \\
& =\left\|\beta_{n}\left(x_{n}-p\right)+\gamma_{n}\left(y_{n}-p\right)+\delta_{n}\left(S y_{n}-p\right)\right\|^{2} \\
& \leq \beta_{n}\left\|x_{n}-p\right\|^{2}+\left(\gamma_{n}+\delta_{n}\right)\left\|y_{n}-p\right\|^{2} \\
& =\beta_{n}\left\|x_{n}-p\right\|^{2}+\left(1-\beta_{n}\right)\left\|y_{n}-p\right\|^{2} \\
& \leq \beta_{n}\left\|x_{n}-p\right\|^{2} \\
& +\left(1-\beta_{n}\right)\left[\sigma_{n}\left\|Q x_{n}-p\right\|^{2}+\left(1-\sigma_{n}\right)\left\|\bar{u}_{n}-p\right\|^{2}\right] \\
& \leq \beta_{n}\left\|x_{n}-p\right\|^{2}+\sigma_{n}\left\|Q x_{n}-p\right\|^{2} \\
& +\left(1-\beta_{n}\right)\left\|\bar{u}_{n}-p\right\|^{2} \\
& \quad+\beta_{n}\left\|x_{n}-p\right\|^{2}+\sigma_{n}\left\|Q x_{n}-p\right\|^{2} \\
& +\left(1-\beta_{n}\right)\left[\left\|x_{n}-p\right\|^{2}-\left\|x_{n}-\tilde{x}_{n}-(p-q)\right\|^{2}\right. \\
& \quad+2 \mu\left\|x_{n}-\tilde{x}_{n}-(p-q)\right\|\left\|B_{2} x_{n}-B_{2} p\right\| \\
& \quad-\left\|\tilde{x}_{n}-u_{n}+(p-q)\right\|^{2} \\
& \quad+2 \mu_{1}\left\|\tilde{x}_{n}-u_{n}+(p-q)\right\|\left\|B_{1} \tilde{x}_{n}-B_{1} q\right\| \\
& \quad+2 \lambda_{n} \alpha_{n}\|p\|\left\|\tilde{u}_{n}-p\right\|
\end{aligned}
$$

which hence implies that

$$
\begin{aligned}
\left(1-\beta_{n}\right)\left[\left\|x_{n}-\tilde{x}_{n}-(p-q)\right\|^{2}+\left\|\tilde{x}_{n}-u_{n}+(p-q)\right\|^{2}\right] \\
\leq\left\|x_{n}-p\right\|^{2}-\left\|x_{n+1}-p\right\|^{2}+\sigma_{n}\left\|Q x_{n}-p\right\|^{2} \\
+\left(1-\beta_{n}\right)\left[2 \mu_{2}\left\|x_{n}-\tilde{x}_{n}-(p-q)\right\|\left\|B_{2} x_{n}-B_{2} p\right\|\right. \\
+2 \mu_{1}\left\|\tilde{x}_{n}-u_{n}+(p-q)\right\|\left\|B_{1} \tilde{x}_{n}-B_{1} q\right\| \\
+2 \lambda_{n} \alpha_{n}\|p\|\left\|\tilde{u}_{n}-p\right\| \\
\left.+2\left\|\bar{u}_{n}-\widetilde{u}_{n}\right\|\left\|\bar{u}_{n}-p\right\|\right]
\end{aligned}
$$




$$
\begin{aligned}
\leq & \left(\left\|x_{n}-p\right\|+\left\|x_{n+1}-p\right\|\right) \\
& \times\left\|x_{n}-x_{n+1}\right\|+\sigma_{n}\left\|Q x_{n}-p\right\|^{2} \\
& +2 \mu_{2}\left\|x_{n}-\widetilde{x}_{n}-(p-q)\right\|\left\|B_{2} x_{n}-B_{2} p\right\| \\
& +2 \mu_{1}\left\|\widetilde{x}_{n}-u_{n}+(p-q)\right\|\left\|B_{1} \widetilde{x}_{n}-B_{1} q\right\| \\
& +2 \lambda_{n} \alpha_{n}\|p\|\left\|\widetilde{u}_{n}-p\right\|+2\left\|\bar{u}_{n}-\widetilde{u}_{n}\right\|\left\|\bar{u}_{n}-p\right\| .
\end{aligned}
$$

Since $\lim \sup _{n \rightarrow \infty} \beta_{n}<1,\left\{\lambda_{n}\right\} \subset[a, b], \alpha_{n} \rightarrow 0, \sigma_{n} \rightarrow$ $0,\left\|B_{2} x_{n}-B_{2} p\right\| \rightarrow 0,\left\|B_{1} \tilde{x}_{n}-B_{1} q\right\| \rightarrow 0,\left\|\bar{u}_{n}-\tilde{u}_{n}\right\| \rightarrow 0$ and $\left\|x_{n+1}-x_{n}\right\| \rightarrow 0$, it follows from the boundedness of $\left\{x_{n}\right\},\left\{\tilde{x}_{n}\right\},\left\{u_{n}\right\},\left\{\tilde{u}_{n}\right\}$, and $\left\{\bar{u}_{n}\right\}$ that

$$
\begin{aligned}
& \lim _{n \rightarrow \infty}\left\|x_{n}-\tilde{x}_{n}-(p-q)\right\|=0, \\
& \lim _{n \rightarrow \infty}\left\|\tilde{x}_{n}-u_{n}+(p-q)\right\|=0 .
\end{aligned}
$$

Consequently, it immediately follows that

$$
\begin{aligned}
& \lim _{n \rightarrow \infty}\left\|x_{n}-u_{n}\right\|=0, \\
& \lim _{n \rightarrow \infty}\left\|x_{n}-\bar{u}_{n}\right\|=0 .
\end{aligned}
$$

This together with $\left\|y_{n}-\bar{u}_{n}\right\| \leq \sigma_{n}\left\|Q x_{n}-\bar{u}_{n}\right\| \rightarrow 0$ implies that

$$
\lim _{n \rightarrow \infty}\left\|x_{n}-y_{n}\right\|=0
$$

Since

$$
\left\|\delta_{n}\left(S y_{n}-x_{n}\right)\right\| \leq\left\|x_{n+1}-x_{n}\right\|+\gamma_{n}\left\|y_{n}-x_{n}\right\|,
$$

it follows that

$$
\lim _{n \rightarrow \infty}\left\|S y_{n}-x_{n}\right\|=0, \quad \lim _{n \rightarrow \infty}\left\|S y_{n}-y_{n}\right\|=0 .
$$

Step 5. $\lim \sup _{n \rightarrow \infty}\left\langle Q \bar{x}-\bar{x}, x_{n}-\bar{x}\right\rangle \leq 0$ where $\bar{x}=$ $P_{\mathrm{Fix}(S) \cap \Xi \cap \Gamma} Q \bar{x}$.

Indeed, since $\left\{x_{n}\right\}$ is bounded, there exists a subsequence $\left\{x_{n_{i}}\right\}$ of $\left\{x_{n}\right\}$ such that

$$
\limsup _{n \rightarrow \infty}\left\langle Q \bar{x}-\bar{x}, x_{n}-\bar{x}\right\rangle=\lim _{i \rightarrow \infty}\left\langle Q \bar{x}-\bar{x}, x_{n_{i}}-\bar{x}\right\rangle .
$$

Also, since $H$ is reflexive and $\left\{y_{n}\right\}$ is bounded, without loss of generality we may assume that $y_{n_{i}} \rightarrow \widehat{p}$ weakly for some $\widehat{p} \in C$. First, it is clear from Lemma 15 that $\widehat{p} \in \operatorname{Fix}(S)$. Now let us show that $\widehat{p} \in \Xi$. We note that

$$
\begin{aligned}
& \left\|x_{n}-G\left(x_{n}\right)\right\| \\
& \quad=\left\|x_{n}-P_{C}\left[P_{C}\left(x_{n}-\mu_{2} B_{2} x_{n}\right)-\mu_{1} B_{1} P_{C}\left(x_{n}-\mu_{2} B_{2} x_{n}\right)\right]\right\| \\
& \quad=\left\|x_{n}-u_{n}\right\| \longrightarrow 0 \quad(n \longrightarrow \infty),
\end{aligned}
$$

where $G: C \rightarrow C$ is defined as such that in Lemma 4 . According to Lemma 15 we obtain $\widehat{p} \in \Xi$. Further, let us show that $\widehat{p} \in \Gamma$. As a matter of fact, since $\left\|x_{n}-u_{n}\right\| \rightarrow$ $0,\left\|\widetilde{u}_{n}-u_{n}\right\| \rightarrow 0$ and $\left\|x_{n}-y_{n}\right\| \rightarrow 0$, we deduce that $x_{n_{i}} \rightarrow \widehat{p}$ weakly and $\tilde{u}_{n_{i}} \rightarrow \widehat{p}$ weakly. Let

$$
T v=\left\{\begin{array}{cl}
\nabla f(v)+N_{C} v, & \text { if } v \in C, \\
\emptyset, & \text { if } v \notin C,
\end{array}\right.
$$

where $N_{C} v=\left\{w \in \mathscr{H}_{1}:\langle v-u, w\rangle \geq 0, \forall u \in C\right\}$. Then, $T$ is maximal monotone and $0 \in T v$ if and only if $v \in \operatorname{VI}(C, \nabla f)$; see [40] for more details. Let $(v, w) \in \operatorname{Gph}(T)$. Then, we have

$$
w \in T v=\nabla f(v)+N_{C} v,
$$

and hence

$$
w-\nabla f(v) \in N_{C} v
$$

So, we have

$$
\langle v-u, w-\nabla f(v)\rangle \geq 0, \quad \forall u \in C .
$$

On the other hand, from

$$
\tilde{u}_{n}=P_{C}\left(u_{n}-\lambda_{n} \nabla f_{\alpha_{n}}\left(u_{n}\right)\right), \quad v \in C,
$$

we have

$$
\left\langle u_{n}-\lambda_{n} \nabla f_{\alpha_{n}}\left(u_{n}\right)-\tilde{u}_{n}, \tilde{u}_{n}-v\right\rangle \geq 0,
$$

and, hence,

$$
\left\langle v-\tilde{u}_{n}, \frac{\tilde{u}_{n}-u_{n}}{\lambda_{n}}+\nabla f_{\alpha_{n}}\left(u_{n}\right)\right\rangle \geq 0 .
$$

Therefore, from

$$
w-\nabla f(v) \in N_{C} v, \quad \tilde{u}_{n_{i}} \in C,
$$

we have

$$
\begin{aligned}
\left\langle v-\tilde{u}_{n_{i}}, w\right\rangle \geq & \left\langle v-\tilde{u}_{n_{i}}, \nabla f(v)\right\rangle \\
\geq & \left\langle v-\tilde{u}_{n_{i}}, \nabla f(v)\right\rangle \\
& -\left\langle v-\tilde{u}_{n_{i}}, \frac{\tilde{u}_{n_{i}}-u_{n_{i}}}{\lambda_{n_{i}}}+\nabla f_{\alpha_{n_{i}}}\left(u_{n_{i}}\right)\right\rangle \\
= & \left\langle v-\tilde{u}_{n_{i}}, \nabla f(v)\right\rangle \\
& -\left\langle v-\tilde{u}_{n_{i}}, \frac{\tilde{u}_{n_{i}}-u_{n_{i}}}{\lambda_{n_{i}}}+\nabla f\left(u_{n_{i}}\right)\right\rangle \\
& -\alpha_{n_{i}}\left\langle v-\tilde{u}_{n_{i}}, u_{n_{i}}\right\rangle \\
= & \left\langle v-\tilde{u}_{n_{i}}, \nabla f(v)-\nabla f\left(\tilde{u}_{n_{i}}\right)\right\rangle \\
& +\left\langle v-\tilde{u}_{n_{i}}, \nabla f\left(\tilde{u}_{n_{i}}\right)-\nabla f\left(u_{n_{i}}\right)\right\rangle \\
& -\left\langle v-\tilde{u}_{n_{i}}, \frac{\tilde{u}_{n_{i}}-u_{n_{i}}}{\lambda_{n_{i}}}\right\rangle-\alpha_{n_{i}}\left\langle v-\tilde{u}_{n_{i}}, u_{n_{i}}\right\rangle \\
\geq & \left\langle v-\tilde{u}_{n_{i}}, \nabla f\left(\tilde{u}_{n_{i}}\right)-\nabla f\left(u_{n_{i}}\right)\right\rangle \\
& -\left\langle v-\tilde{u}_{n_{i}}, \frac{\tilde{u}_{n_{i}}-u_{n_{i}}}{\lambda_{n_{i}}}\right\rangle-\alpha_{n_{i}}\left\langle v-\tilde{u}_{n_{i}}, u_{n_{i}}\right\rangle .
\end{aligned}
$$


Hence, we get

$$
\langle v-\widehat{p}, w\rangle \geq 0, \quad \text { as } i \longrightarrow \infty
$$

Since $T$ is maximal monotone, we have $\widehat{p} \in T^{-1} 0$, and, hence, $\widehat{p} \in \operatorname{VI}(C, \nabla f)$. Thus it is clear that $\widehat{p} \in \Gamma$. Therefore, $\widehat{p} \in$ $\operatorname{Fix}(S) \cap \Xi \cap \Gamma$. Consequently, in terms of Proposition 8(i) we obtain from (84) that

$$
\begin{aligned}
& \limsup _{n \rightarrow \infty}\left\langle Q \bar{x}-\bar{x}, x_{n}-\bar{x}\right\rangle \\
& \quad=\lim _{i \rightarrow \infty}\left\langle Q \bar{x}-\bar{x}, x_{n_{i}}-\bar{x}\right\rangle=\langle Q \bar{x}-\bar{x}, \widehat{p}-\bar{x}\rangle \leq 0 .
\end{aligned}
$$

Step 6. $\lim _{n \rightarrow \infty}\left\|x_{n}-\bar{x}\right\|=0$.

Indeed, from (48) and (51) it follows that

$$
\begin{aligned}
\left\|\bar{u}_{n}-\bar{x}\right\|^{2} & \leq\left\|u_{n}-\bar{x}\right\|^{2}+2 \lambda_{n} \alpha_{n}\|\bar{x}\|\left\|\tilde{u}_{n}-\bar{x}\right\| \\
& \leq\left\|x_{n}-\bar{x}\right\|^{2}+2 \lambda_{n} \alpha_{n}\|\bar{x}\|\left\|\tilde{u}_{n}-\bar{x}\right\| .
\end{aligned}
$$

Note that

$$
\begin{aligned}
\left\langle Q x_{n}-\bar{x}, y_{n}-\bar{x}\right\rangle= & \left\langle Q x_{n}-\bar{x}, x_{n}-\bar{x}\right\rangle \\
& +\left\langle Q x_{n}-\bar{x}, y_{n}-x_{n}\right\rangle \\
= & \left\langle Q x_{n}-Q \bar{x}, x_{n}-\bar{x}\right\rangle \\
& +\left\langle Q \bar{x}-\bar{x}, x_{n}-\bar{x}\right\rangle \\
& +\left\langle Q x_{n}-\bar{x}, y_{n}-x_{n}\right\rangle \\
\leq & \rho\left\|x_{n}-\bar{x}\right\|^{2}+\left\langle Q \bar{x}-\bar{x}, x_{n}-\bar{x}\right\rangle \\
& +\left\|Q x_{n}-\bar{x}\right\|\left\|y_{n}-x_{n}\right\| .
\end{aligned}
$$

Utilizing Lemmas 17 and 18, we obtain from (48) and the convexity of $\|\cdot\|^{2}$

$$
\begin{aligned}
& \left\|x_{n+1}-\bar{x}\right\|^{2} \\
& =\left\|\beta_{n}\left(x_{n}-\bar{x}\right)+\gamma_{n}\left(y_{n}-\bar{x}\right)+\delta_{n}\left(S y_{n}-\bar{x}\right)\right\|^{2} \\
& \leq \beta_{n}\left\|x_{n}-\bar{x}\right\|^{2} \\
& \quad+\left(\gamma_{n}+\delta_{n}\right)\left\|\frac{1}{\gamma_{n}+\delta_{n}}\left[\gamma_{n}\left(y_{n}-\bar{x}\right)+\delta_{n}\left(S y_{n}-\bar{x}\right)\right]\right\|^{2} \\
& \leq \beta_{n}\left\|x_{n}-\bar{x}\right\|^{2}+\left(\gamma_{n}+\delta_{n}\right)\left\|y_{n}-\bar{x}\right\|^{2} \\
& \leq \beta_{n}\left\|x_{n}-\bar{x}\right\|^{2}+\left(\gamma_{n}+\delta_{n}\right)\left[\left(1-\sigma_{n}\right)^{2}\left\|\bar{u}_{n}-\bar{x}\right\|^{2}\right. \\
& \left.\quad+2 \sigma_{n}\left\langle Q x_{n}-\bar{x}, y_{n}-\bar{x}\right\rangle\right]
\end{aligned}
$$

$$
\begin{aligned}
& \leq \beta_{n}\left\|x_{n}-\bar{x}\right\|^{2} \\
& +\left(\gamma_{n}+\delta_{n}\right)\left[\left(1-\sigma_{n}\right)\right. \\
& \times\left(\left\|x_{n}-\bar{x}\right\|^{2}+2 \lambda_{n} \alpha_{n}\|\bar{x}\|\left\|\tilde{u}_{n}-\bar{x}\right\|\right) \\
& \left.+2 \sigma_{n}\left\langle Q x_{n}-\bar{x}, y_{n}-\bar{x}\right\rangle\right] \\
& =\left(1-\left(\gamma_{n}+\delta_{n}\right) \sigma_{n}\right)\left\|x_{n}-\bar{x}\right\|^{2} \\
& +\left(\gamma_{n}+\delta_{n}\right) 2 \sigma_{n}\left\langle Q x_{n}-\bar{x}, y_{n}-\bar{x}\right\rangle \\
& +\left(\gamma_{n}+\delta_{n}\right) 2 \lambda_{n} \alpha_{n}\|\bar{x}\|\left\|\tilde{u}_{n}-\bar{x}\right\| \\
& \leq\left(1-\left(\gamma_{n}+\delta_{n}\right) \sigma_{n}\right)\left\|x_{n}-\bar{x}\right\|^{2} \\
& +\left(\gamma_{n}+\delta_{n}\right) 2 \sigma_{n}\left\langle Q x_{n}-\bar{x}, y_{n}-\bar{x}\right\rangle \\
& +2 \lambda_{n} \alpha_{n}\|\bar{x}\|\left\|\tilde{u}_{n}-\bar{x}\right\| \\
& \leq\left(1-\left(\gamma_{n}+\delta_{n}\right) \sigma_{n}\right)\left\|x_{n}-\bar{x}\right\|^{2}+\left(\gamma_{n}+\delta_{n}\right) 2 \sigma_{n} \\
& \times\left[\rho\left\|x_{n}-\bar{x}\right\|^{2}\right. \\
& \left.+\left\langle Q \bar{x}-\bar{x}, x_{n}-\bar{x}\right\rangle+\left\|Q x_{n}-\bar{x}\right\|\left\|y_{n}-x_{n}\right\|\right] \\
& +2 \lambda_{n} \alpha_{n}\|\bar{x}\|\left\|\tilde{u}_{n}-\bar{x}\right\| \\
& =\left[1-(1-2 \rho)\left(\gamma_{n}+\delta_{n}\right) \sigma_{n}\right]\left\|x_{n}-\bar{x}\right\|^{2}+\left(\gamma_{n}+\delta_{n}\right) 2 \sigma_{n} \\
& \times\left[\left\langle Q \bar{x}-\bar{x}, x_{n}-\bar{x}\right\rangle+\left\|Q x_{n}-\bar{x}\right\|\left\|y_{n}-x_{n}\right\|\right] \\
& +2 \lambda_{n} \alpha_{n}\|\bar{x}\|\left\|\tilde{u}_{n}-\bar{x}\right\| \\
& =\left[1-(1-2 \rho)\left(\gamma_{n}+\delta_{n}\right) \sigma_{n}\right]\left\|x_{n}-\bar{x}\right\|^{2}+(1-2 \rho) \\
& \times \sigma_{n} \frac{2\left[\left\langle Q \bar{x}-\bar{x}, x_{n}-\bar{x}\right\rangle+\left\|Q x_{n}-\bar{x}\right\|\left\|y_{n}-x_{n}\right\|\right]}{1-2 \rho} \\
& +2 \lambda_{n} \alpha_{n}\|\bar{x}\|\left\|\tilde{u}_{n}-\bar{x}\right\| \text {. }
\end{aligned}
$$

Note that $\liminf _{n \rightarrow \infty}(1-2 \rho)\left(\gamma_{n}+\delta_{n}\right)>0$. It follows that $\sum_{n=0}^{\infty}(1-2 \rho)\left(\gamma_{n}+\delta_{n}\right) \sigma_{n}=\infty$. It is clear that

$$
\limsup _{n \rightarrow \infty} \frac{2\left[\left\langle Q \bar{x}-\bar{x}, x_{n}-\bar{x}\right\rangle+\left\|Q x_{n}-\bar{x}\right\|\left\|y_{n}-x_{n}\right\|\right]}{1-2 \rho} \leq 0,
$$

because $\limsup _{n \rightarrow \infty}\left\langle Q \bar{x}-\bar{x}, x_{n}-\bar{x}\right\rangle \leq 0$ and $\lim _{n \rightarrow \infty} \| x_{n}-$ $y_{n} \|=0$. In addition, note also that $\left\{\lambda_{n}\right\} \subset[a, b], \sum_{n=0}^{\infty} \alpha_{n}<$ $\infty$ and $\left\{\tilde{u}_{n}\right\}$ is bounded. Hence we get $\sum_{n=0}^{\infty} 2 \lambda_{n} \alpha_{n}\|\bar{x}\| \| \widetilde{u}_{n}-$ $\bar{x} \|<\infty$. Therefore, all conditions of Lemma 16 are satisfied. Consequently, we immediately deduce that $\left\|x_{n}-\bar{x}\right\| \rightarrow 0$ as $n \rightarrow \infty$. This completes the proof.

Corollary 20. Let $C$ be a nonempty closed convex subset of a real Hilbert space $\mathscr{H}_{1}$. Let $A \in B\left(\mathscr{H}_{1}, \mathscr{H}_{2}\right)$ and $B_{i}: C \rightarrow \mathscr{H}_{1}$ be $\beta_{i}$-inverse strongly monotone for $i=1,2$. Let $S: C \rightarrow C$ 
be a $k$-strictly pseudo-contractive mapping such that Fix $(S) \cap$ $\Xi \cap \Gamma \neq \emptyset$. For fixed $u \in C$ and given $x_{0} \in C$ arbitrarily, let the sequences $\left\{x_{n}\right\},\left\{u_{n}\right\},\left\{\tilde{u}_{n}\right\}$ be generated iteratively by

$$
\begin{gathered}
u_{n}=P_{C}\left[P_{C}\left(x_{n}-\mu_{2} B_{2} x_{n}\right)-\mu_{1} B_{1} P_{C}\left(x_{n}-\mu_{2} B_{2} x_{n}\right)\right], \\
\tilde{u}_{n}=P_{C}\left(u_{n}-\lambda_{n} \nabla f_{\alpha_{n}}\left(u_{n}\right)\right), \\
y_{n}=\sigma_{n} u+\left(1-\sigma_{n}\right) P_{C}\left(u_{n}-\lambda_{n} \nabla f_{\alpha_{n}}\left(\widetilde{u}_{n}\right)\right), \\
x_{n+1}=\beta_{n} x_{n}+\gamma_{n} y_{n}+\delta_{n} S y_{n}, \quad \forall n \geq 0,
\end{gathered}
$$

where $\mu_{i} \in\left(0,2 \beta_{i}\right)$ for $i=1,2$, and the following conditions hold for six sequences $\left\{\alpha_{n}\right\} \subset(0, \infty),\left\{\lambda_{n}\right\} \subset\left(0,1 /\|A\|^{2}\right)$ and $\left\{\sigma_{n}\right\},\left\{\beta_{n}\right\},\left\{\gamma_{n}\right\},\left\{\delta_{n}\right\} \subset[0,1]$ :

(i) $\sum_{n=0}^{\infty} \alpha_{n}<\infty$;

(ii) $\beta_{n}+\gamma_{n}+\delta_{n}=1$ and $\left(\gamma_{n}+\delta_{n}\right) k \leq \gamma_{n}$ for all $n \geq 0$;

(iii) $\lim _{n \rightarrow \infty} \sigma_{n}=0$ and $\sum_{n=0}^{\infty} \sigma_{n}=\infty$;

(iv) $0<\liminf _{n \rightarrow \infty} \beta_{n} \leq \limsup _{n \rightarrow \infty} \beta_{n}<1$ and $\liminf _{n \rightarrow \infty} \delta_{n}>0$;

(v) $\lim _{n \rightarrow \infty}\left(\gamma_{n+1} /\left(1-\beta_{n+1}\right)-\gamma_{n} /\left(1-\beta_{n}\right)\right)=0$;

(vi) $0<\liminf _{n \rightarrow \infty} \lambda_{n} \leq \limsup _{n \rightarrow \infty} \lambda_{n}<1 /\|A\|^{2}$ and $\lim _{n \rightarrow \infty}\left|\lambda_{n+1}-\lambda_{n}\right|=0$.

Then the sequences $\left\{x_{n}\right\},\left\{u_{n}\right\},\left\{\tilde{u}_{n}\right\}$ converge strongly to the same point $\bar{x}=P_{\text {Fix }(S) \cap \Xi \cap \Gamma} u$ if and only if $\lim _{n \rightarrow \infty} \| u_{n+1}-$ $u_{n} \|=0$. Furthermore, $(\bar{x}, \bar{y})$ is a solution of the GSVI (14), where $\bar{y}=P_{C}\left(\bar{x}-\mu_{2} B_{2} \bar{x}\right)$.

Next, utilizing Corollary 20 we give the following improvement and extension of the main result in [18] (i.e., [18, Theorem 3.1]).

Corollary 21. Let $C$ be a nonempty closed convex subset of a real Hilbert space $\mathscr{H}_{1}$. Let $A \in B\left(\mathscr{H}_{1}, \mathscr{H}_{2}\right)$ and $S: C \rightarrow C$ be a nonexpansive mapping such that Fix $(S) \cap \Gamma \neq \emptyset$. For fixed $u \in C$ and given $x_{0} \in C$ arbitrarily, let the sequences $\left\{u_{n}\right\},\left\{\tilde{u}_{n}\right\}$ be generated iteratively by

$$
\begin{aligned}
\tilde{u}_{n}= & P_{C}\left(u_{n}-\lambda_{n} \nabla f_{\alpha_{n}}\left(u_{n}\right)\right), \\
u_{n+1}= & \beta_{n} u_{n}+\left(1-\beta_{n}\right) \\
\times S\left[\sigma_{n} u+\left(1-\sigma_{n}\right) P_{C}\right. & \\
& \left.\left(u_{n}-\lambda_{n} \nabla f_{\alpha_{n}}\left(\widetilde{u}_{n}\right)\right)\right], \quad \forall n \geq 0,
\end{aligned}
$$

where the following conditions hold for four sequences $\left\{\alpha_{n}\right\} \subset$ $(0, \infty),\left\{\lambda_{n}\right\} \subset\left(0,1 /\|A\|^{2}\right)$ and $\left\{\sigma_{n}\right\},\left\{\beta_{n}\right\} \subset[0,1]$ :

(i) $\sum_{n=0}^{\infty} \alpha_{n}<\infty$;

(ii) $\lim _{n \rightarrow \infty} \sigma_{n}=0$ and $\sum_{n=0}^{\infty} \sigma_{n}=\infty$;

(iii) $0<\liminf _{n \rightarrow \infty} \beta_{n} \leq \lim \sup _{n \rightarrow \infty} \beta_{n}<1$;

(iv) $0<\liminf _{n \rightarrow \infty} \lambda_{n} \leq \limsup _{n \rightarrow \infty} \lambda_{n}<1 /\|A\|^{2}$ and $\lim _{n \rightarrow \infty}\left|\lambda_{n+1}-\lambda_{n}\right|=0$.

Then the sequences $\left\{u_{n}\right\},\left\{\widetilde{u}_{n}\right\}$ converge strongly to the same point $\bar{x}=P_{\text {Fix }(S) \cap \Gamma} u$ if and only if $\lim _{n \rightarrow \infty}\left\|u_{n+1}-u_{n}\right\|=0$.
Proof. In Corollary 20, put $B_{1}=B_{2}=0$ and $\gamma_{n}=0$. Then, $\Xi=C, \beta_{n}+\delta_{n}=1$ for all $n \geq 0$, and the iterative scheme (101) is equivalent to

$$
\begin{gathered}
u_{n}=x_{n}, \\
\tilde{u}_{n}=P_{C}\left(u_{n}-\lambda_{n} \nabla f_{\alpha_{n}}\left(u_{n}\right)\right), \\
y_{n}=\sigma_{n} u+\left(1-\sigma_{n}\right) P_{C}\left(u_{n}-\lambda_{n} \nabla f_{\alpha_{n}}\left(\tilde{u}_{n}\right)\right), \\
u_{n+1}=\beta_{n} x_{n}+\delta_{n} S y_{n}, \quad \forall n \geq 0 .
\end{gathered}
$$

This is is equivalent to (102). Since $S$ is a nonexpansive mapping, $S$ must be a $k$-strictly pseudo-contractive mapping with $k=0$. In this case, it is easy to see that conditions (i)(vi) in Corollary 20 all are satisfied. Therefore, in terms of Corollary 20, we obtain the desired result.

Now, we are in a position to prove the strong convergence of the sequences generated by the relaxed extragradient iterative algorithm (18) with regularization.

Theorem 22. Let $C$ be a nonempty closed convex subset of a real Hilbert space $\mathscr{H}_{1}$. Let $A \in B\left(\mathscr{H}_{1}, \mathscr{H}_{2}\right)$ and $B_{i}: C \rightarrow \mathscr{H}_{1}$ be $\beta_{i}$-inverse strongly monotone for $i=1,2$. Let $S: C \rightarrow C$ be a $k$-strictly pseudocontractive mapping such that Fix $(S) \cap \Xi \cap$ $\Gamma \neq \emptyset$. Let $Q: C \rightarrow C$ be a $\rho$-contraction with $\rho \in[0,1 / 2)$. For given $x_{0} \in C$ arbitrarily, let the sequences $\left\{x_{n}\right\},\left\{y_{n}\right\},\left\{z_{n}\right\}$ be generated by the relaxed extragradient iterative algorithm (18) with regularization, where $\mu_{i} \in\left(0,2 \beta_{i}\right)$ for $i=1,2,\left\{\alpha_{n}\right\} \subset$ $(0, \infty),\left\{\lambda_{n}\right\} \subset\left(0,1 /\|A\|^{2}\right)$ and $\left\{\sigma_{n}\right\},\left\{\tau_{n}\right\},\left\{\beta_{n}\right\},\left\{\gamma_{n}\right\},\left\{\delta_{n}\right\} \subset$ $[0,1]$ such that

(i) $\sum_{n=0}^{\infty} \alpha_{n}<\infty$;

(ii) $\sigma_{n}+\tau_{n} \leq 1, \beta_{n}+\gamma_{n}+\delta_{n}=1$ and $\left(\gamma_{n}+\delta_{n}\right) k \leq \gamma_{n}$ for all $n \geq 0$;

(iii) $\lim _{n \rightarrow \infty} \sigma_{n}=0$ and $\sum_{n=0}^{\infty} \sigma_{n}=\infty$;

(iv) $0<\liminf _{n \rightarrow \infty} \tau_{n} \leq \limsup _{n \rightarrow \infty} \tau_{n}<1$ and $\lim _{n \rightarrow \infty}\left|\tau_{n+1}-\tau_{n}\right|=0$;

(v) $0<\liminf _{n \rightarrow \infty} \beta_{n} \leq \limsup _{n \rightarrow \infty} \beta_{n}<1$ and $\liminf _{n \rightarrow \infty} \delta_{n}>0$;

(vi) $\lim _{n \rightarrow \infty}\left(\gamma_{n+1} /\left(1-\beta_{n+1}\right)-\gamma_{n} /\left(1-\beta_{n}\right)\right)=0$;

(vii) $0<\liminf _{n \rightarrow \infty} \lambda_{n} \leq \limsup _{n \rightarrow \infty} \lambda_{n}<1 /\|A\|^{2}$ and $\lim _{n \rightarrow \infty}\left|\lambda_{n+1}-\lambda_{n}\right|=0$.

Then the sequences $\left\{x_{n}\right\},\left\{y_{n}\right\},\left\{z_{n}\right\}$ converge strongly to the same point $\bar{x}=P_{\text {Fix }(S) \cap \Xi \cap \Gamma} Q \bar{x}$ if and only if $\lim _{n \rightarrow \infty} \| z_{n+1}-$ $z_{n} \|=0$. Furthermore, $(\bar{x}, \bar{y})$ is a solution of the GSVI (14), where $\bar{y}=P_{C}\left(\bar{x}-\mu_{2} B_{2} \bar{x}\right)$.

Proof. First, taking into account $0<\liminf _{n \rightarrow \infty} \lambda_{n} \leq$ $\limsup _{n \rightarrow \infty} \lambda_{n}<1 /\|A\|^{2}$, without loss of generality we may assume that $\left\{\lambda_{n}\right\} \subset[a, b]$ for some $a, b \in\left(0,1 /\|A\|^{2}\right)$. Repeating the same argument as that in the proof of Theorem 19, we can show that $P_{C}\left(I-\lambda \nabla f_{\alpha}\right)$ is $\zeta$-averaged for each $\lambda \in\left(0,2 /\left(\alpha+\|A\|^{2}\right)\right)$, where $\zeta=\left(2+\lambda\left(\alpha+\|A\|^{2}\right)\right) / 4$. Further, repeating the same argument as that in the proof of Theorem 19, we can also show that for each integer $n \geq$ 
$0, P_{C}\left(I-\lambda_{n} \nabla f_{\alpha_{n}}\right)$ is $\zeta_{n}$-averaged with $\zeta_{n}=\left(2+\lambda_{n}\left(\alpha_{n}+\right.\right.$ $\left.\left.\|A\|^{2}\right)\right) / 4 \in(0,1)$.

Next we divide the remainder of the proof into several steps.

Step 1. $\left\{x_{n}\right\}$ is bounded.

Indeed, take $p \in \operatorname{Fix}(S) \cap \Xi \cap \Gamma$ arbitrarily. Then $S p=$ $p, P_{C}(I-\lambda \nabla f) p=p$ for $\lambda \in\left(0,2 /\|A\|^{2}\right)$, and

$$
p=P_{C}\left[P_{C}\left(p-\mu_{2} B_{2} p\right)-\mu_{1} B_{1} P_{C}\left(p-\mu_{2} B_{2} p\right)\right] .
$$

Utilizing the arguments similar to those of (45) and (46) in the proof of Theorem 19, from (18) we can obtain

$$
\begin{gathered}
\left\|z_{n}-p\right\| \leq\left\|x_{n}-p\right\|+\lambda_{n} \alpha_{n}\|p\|, \\
\left\|z_{n}-p\right\|^{2} \leq\left\|x_{n}-p\right\|^{2}+2 \lambda_{n} \alpha_{n}\|p\|\left\|z_{n}-p\right\| .
\end{gathered}
$$

For simplicity, we write $q=P_{C}\left(p-\mu_{2} B_{2} p\right), \widetilde{z}_{n}=P_{C}\left(z_{n}-\right.$ $\left.\mu_{2} B_{2} z_{n}\right)$,

$$
\begin{gathered}
u_{n}=P_{C}\left[P_{C}\left(z_{n}-\mu_{2} B_{2} z_{n}\right)\right. \\
\left.-\mu_{1} B_{1} P_{C}\left(z_{n}-\mu_{2} B_{2} z_{n}\right)\right], \\
\bar{u}_{n}=P_{C}\left(x_{n}-\lambda_{n} \nabla f_{\alpha_{n}}\left(z_{n}\right)\right),
\end{gathered}
$$

for each $n \geq 0$. Then $y_{n}=\sigma_{n} Q x_{n}+\tau_{n} \bar{u}_{n}+\left(1-\sigma_{n}-\tau_{n}\right) u_{n}$ for each $n \geq 0$. Since $B_{i}: C \rightarrow \mathscr{H}_{1}$ is $\beta_{i}$-inverse strongly monotone and $0<\mu_{i}<2 \beta_{i}$ for $i=1,2$, utilizing the argument similar to that of (48) in the proof of Theorem 19, we can obtain that for all $n \geq 0$,

$$
\begin{aligned}
\left\|u_{n}-p\right\|^{2} \leq & \left\|z_{n}-p\right\|^{2}-\mu_{2}\left(2 \beta_{2}-\mu_{2}\right)\left\|B_{2} z_{n}-B_{2} p\right\|^{2} \\
& -\mu_{1}\left(2 \beta_{1}-\mu_{1}\right)\left\|B_{1} \widetilde{z}_{n}-B_{1} q\right\|^{2} \leq\left\|z_{n}-p\right\|^{2} .
\end{aligned}
$$

Furthermore, utilizing Proposition 8(i)-(ii) and the argument similar to that of (51) in the proof of Theorem 19, from (105) we obtain

$$
\begin{aligned}
&\left\|\bar{u}_{n}-p\right\|^{2} \\
& \leq\left\|x_{n}-p\right\|^{2}+2 \lambda_{n} \alpha_{n}\|p\|\left\|z_{n}-p\right\| \\
&+\left(\lambda_{n}^{2}\left(\alpha_{n}+\|A\|^{2}\right)^{2}-1\right)\left\|x_{n}-z_{n}\right\|^{2} \\
& \leq\left\|x_{n}-p\right\|^{2}+2 \lambda_{n} \alpha_{n}\|p\|\left\|z_{n}-p\right\| \\
& \leq\left\|x_{n}-p\right\|^{2}+2 \lambda_{n} \alpha_{n}\|p\|\left[\left\|x_{n}-p\right\|+\lambda_{n} \alpha_{n}\|p\|\right] \\
& \leq\left\|x_{n}-p\right\|^{2}+4 \lambda_{n} \alpha_{n}\|p\|\left\|x_{n}-p\right\|+4 \lambda_{n}^{2} \alpha_{n}^{2}\|p\|^{2} \\
&=\left(\left\|x_{n}-p\right\|+2 \lambda_{n} \alpha_{n}\|p\|\right)^{2} .
\end{aligned}
$$

Hence it follows from (105), (108), and (109) that

$$
\begin{aligned}
& \left\|y_{n}-p\right\| \\
& =\| \sigma_{n}\left(Q x_{n}-p\right) \\
& +\tau_{n}\left(\bar{u}_{n}-p\right)+\left(1-\sigma_{n}-\tau_{n}\right)\left(u_{n}-p\right) \| \\
& \leq \sigma_{n}\left\|Q x_{n}-p\right\|+\tau_{n}\left\|\bar{u}_{n}-p\right\| \\
& +\left(1-\sigma_{n}-\tau_{n}\right)\left\|u_{n}-p\right\| \\
& \leq \sigma_{n}\left(\rho\left\|x_{n}-p\right\|+\|Q p-p\|\right) \\
& +\tau_{n}\left\|\bar{u}_{n}-p\right\|+\left(1-\sigma_{n}-\tau_{n}\right)\left\|z_{n}-p\right\| \\
& \leq \sigma_{n}\left(\rho\left\|x_{n}-p\right\|+\|Q p-p\|\right) \\
& +\tau_{n}\left(\left\|x_{n}-p\right\|+2 \lambda_{n} \alpha_{n}\|p\|\right) \\
& +\left(1-\sigma_{n}-\tau_{n}\right)\left(\left\|x_{n}-p\right\|+\lambda_{n} \alpha_{n}\|p\|\right) \\
& =\left(1-(1-\rho) \sigma_{n}\right)\left\|x_{n}-p\right\|+\sigma_{n}\|Q p-p\| \\
& +\left[2 \tau_{n}+\left(1-\sigma_{n}-\tau_{n}\right)\right] \lambda_{n} \alpha_{n}\|p\| \\
& \leq\left(1-(1-\rho) \sigma_{n}\right)\left\|x_{n}-p\right\| \\
& +\sigma_{n}\|Q p-p\|+2 \lambda_{n} \alpha_{n}\|p\| \\
& =\left(1-(1-\rho) \sigma_{n}\right)\left\|x_{n}-p\right\| \\
& +(1-\rho) \sigma_{n} \frac{\|Q p-p\|}{1-\rho}+2 \lambda_{n} \alpha_{n}\|p\| \\
& \leq \max \left\{\left\|x_{n}-p\right\|, \frac{\|Q p-p\|}{1-\rho}\right\}+2 \lambda_{n} \alpha_{n}\|p\| .
\end{aligned}
$$

Since $\left(\gamma_{n}+\delta_{n}\right) k \leq \gamma_{n}$ for all $n \geq 0$, utilizing Lemma 17 we obtain from (110)

$$
\begin{aligned}
&\left\|x_{n+1}-p\right\| \\
&=\left\|\beta_{n}\left(x_{n}-p\right)+\gamma_{n}\left(y_{n}-p\right)+\delta_{n}\left(S y_{n}-p\right)\right\| \\
& \leq \beta_{n}\left\|x_{n}-p\right\|+\left\|\gamma_{n}\left(y_{n}-p\right)+\delta_{n}\left(S y_{n}-p\right)\right\| \\
& \leq \beta_{n}\left\|x_{n}-p\right\|+\left(\gamma_{n}+\delta_{n}\right)\left\|y_{n}-p\right\| \\
& \leq \beta_{n}\left\|x_{n}-p\right\| \\
&+\left(\gamma_{n}+\delta_{n}\right)\left[\max \left\{\left\|x_{n}-p\right\|, \frac{\|Q p-p\|}{1-\rho}\right\}+2 \lambda_{n} \alpha_{n}\|p\|\right] \\
& \leq \beta_{n}\left\|x_{n}-p\right\| \\
&+\left(\gamma_{n}+\delta_{n}\right) \max \left\{\left\|x_{n}-p\right\|, \frac{\|Q p-p\|}{1-\rho}\right\}+2 \lambda_{n} \alpha_{n}\|p\| \\
& \leq \max \left\{\left\|x_{n}-p\right\|, \frac{\|Q p-p\|}{1-\rho}\right\}+2 b\|p\| \alpha_{n} .
\end{aligned}
$$


Repeating the same argument as that of (54) in the proof of Theorem 19, by induction we can prove that

$$
\left\|x_{n+1}-p\right\| \leq \max \left\{\left\|x_{0}-p\right\|, \frac{\|Q p-p\|}{1-\rho}\right\}+2 b\|p\| \sum_{j=0}^{n} \alpha_{j} .
$$

Thus, $\left\{x_{n}\right\}$ is bounded. Since $P_{C}, \nabla f_{\alpha_{n}}, B_{1}$ and $B_{2}$ are Lipschitz continuous, it is easy to see that $\left\{z_{n}\right\},\left\{u_{n}\right\},\left\{\bar{u}_{n}\right\},\left\{y_{n}\right\}$, and $\left\{\widetilde{z}_{n}\right\}$ are bounded, where $\widetilde{z}_{n}=P_{C}\left(z_{n}-\mu_{2} B_{2} z_{n}\right)$ for all $n \geq 0$.

Step 2. $\lim _{n \rightarrow \infty}\left\|x_{n+1}-x_{n}\right\|=0$.

Indeed, define $x_{n+1}=\beta_{n} x_{n}+\left(1-\beta_{n}\right) w_{n}$ for all $n \geq 0$. Then, utilizing the arguments similar to those of (58)-(61) in the proof of Theorem 19, we can obtain that

$$
\begin{gathered}
w_{n+1}-w_{n}=\frac{\gamma_{n+1}\left(y_{n+1}-y_{n}\right)+\delta_{n+1}\left(S y_{n+1}-S y_{n}\right)}{1-\beta_{n+1}} \\
+\left(\frac{\gamma_{n+1}}{1-\beta_{n+1}}-\frac{\gamma_{n}}{1-\beta_{n}}\right) y_{n} \\
+\left(\frac{\delta_{n+1}}{1-\beta_{n+1}}-\frac{\delta_{n}}{1-\beta_{n}}\right) S y_{n}, \\
\left\|\gamma_{n+1}\left(y_{n+1}-y_{n}\right)+\delta_{n+1}\left(S y_{n+1}-S y_{n}\right)\right\| \\
\leq\left(\gamma_{n+1}+\delta_{n+1}\right)\left\|y_{n+1}-y_{n}\right\|,
\end{gathered}
$$

(due to Lemma 17)

$$
\begin{aligned}
& \left\|z_{n+1}-z_{n}\right\| \leq\left\|x_{n+1}-x_{n}\right\|+\left|\lambda_{n+1}-\lambda_{n}\right|\left\|\nabla f\left(x_{n}\right)\right\| \\
& +\left|\lambda_{n+1} \alpha_{n+1}-\lambda_{n} \alpha_{n}\right|\left\|x_{n}\right\| \\
& \left\|u_{n+1}-u_{n}\right\|^{2} \\
& \leq\left\|P_{C}\left(z_{n+1}-\mu_{2} B_{2} z_{n+1}\right)-P_{C}\left(z_{n}-\mu_{2} B_{2} z_{n}\right)\right\|^{2} \\
& -\mu_{1}\left(2 \beta_{1}-\mu_{1}\right) \| B_{1} P_{C}\left(z_{n+1}-\mu_{2} B_{2} z_{n+1}\right) \\
& -B_{1} P_{C}\left(z_{n}-\mu_{2} B_{2} z_{n}\right) \|^{2} \\
& \leq\left\|\left(z_{n+1}-\mu_{2} B_{2} z_{n+1}\right)-\left(z_{n}-\mu_{2} B_{2} z_{n}\right)\right\|^{2} \\
& \leq\left\|z_{n+1}-z_{n}\right\|^{2} \\
& -\mu_{2}\left(2 \beta_{2}-\mu_{2}\right)\left\|B_{2} z_{n+1}-B_{2} z_{n}\right\|^{2} \\
& \leq\left\|z_{n+1}-z_{n}\right\|^{2} \text {. }
\end{aligned}
$$

So, we have

$$
\begin{aligned}
& \left\|\bar{u}_{n+1}-\bar{u}_{n}\right\| \\
& =\| P_{C}\left(x_{n+1}-\lambda_{n+1} \nabla f_{\alpha_{n+1}}\left(z_{n+1}\right)\right) \\
& \quad-P_{C}\left(x_{n}-\lambda_{n} \nabla f_{\alpha_{n}}\left(z_{n}\right)\right) \| \\
& \leq\left\|\left(x_{n+1}-\lambda_{n+1} \nabla f_{\alpha_{n+1}}\left(z_{n+1}\right)\right)-\left(x_{n}-\lambda_{n} \nabla f_{\alpha_{n}}\left(z_{n}\right)\right)\right\|
\end{aligned}
$$

$$
\begin{aligned}
= & \| x_{n+1}-x_{n}-\lambda_{n+1}\left[\nabla f_{\alpha_{n+1}}\left(z_{n+1}\right)-\nabla f_{\alpha_{n+1}}\left(z_{n}\right)\right] \\
& -\left[\lambda_{n+1} \nabla f_{\alpha_{n+1}}\left(z_{n}\right)-\lambda_{n} \nabla f_{\alpha_{n}}\left(z_{n}\right)\right] \| \\
\leq & \left\|x_{n+1}-x_{n}\right\|+\lambda_{n+1}\left\|\nabla f_{\alpha_{n+1}}\left(z_{n+1}\right)-\nabla f_{\alpha_{n+1}}\left(z_{n}\right)\right\| \\
& +\left\|\lambda_{n+1} \nabla f_{\alpha_{n+1}}\left(z_{n}\right)-\lambda_{n} \nabla f_{\alpha_{n}}\left(z_{n}\right)\right\| \\
\leq & \left\|x_{n+1}-x_{n}\right\|+\lambda_{n+1}\left(\alpha_{n+1}+\|A\|^{2}\right)\left\|z_{n+1}-z_{n}\right\| \\
& +\left\|\lambda_{n+1}\left(\alpha_{n+1} I+\nabla f\right) z_{n}-\lambda_{n}\left(\alpha_{n} I+\nabla f\right) z_{n}\right\| \\
\leq & \left\|x_{n+1}-x_{n}\right\|+\lambda_{n+1}\left(\alpha_{n+1}+\|A\|^{2}\right)\left\|z_{n+1}-z_{n}\right\| \\
& +\left|\lambda_{n+1} \alpha_{n+1}-\lambda_{n} \alpha_{n}\right|\left\|z_{n}\right\|+\left|\lambda_{n+1}-\lambda_{n}\right|\left\|\nabla f\left(z_{n}\right)\right\| \\
\leq & \left\|x_{n+1}-x_{n}\right\|+\left\|z_{n+1}-z_{n}\right\| \\
& +\left|\lambda_{n+1} \alpha_{n+1}-\lambda_{n} \alpha_{n}\right|\left\|z_{n}\right\|+\left|\lambda_{n+1}-\lambda_{n}\right|\left\|\nabla f\left(z_{n}\right)\right\| .
\end{aligned}
$$

This together with (114) implies that

$$
\begin{aligned}
& \left\|y_{n+1}-y_{n}\right\| \\
& =\| \sigma_{n+1}\left(Q x_{n+1}-u_{n+1}\right)+\tau_{n+1} \bar{u}_{n+1}+\left(1-\tau_{n+1}\right) u_{n+1} \\
& -\sigma_{n}\left(Q x_{n}-u_{n}\right)-\tau_{n} \bar{u}_{n}-\left(1-\tau_{n}\right) u_{n} \| \\
& \leq\left\|\tau_{n+1} \bar{u}_{n+1}-\tau_{n} \bar{u}_{n}\right\|+\left\|\left(1-\tau_{n+1}\right) u_{n+1}-\left(1-\tau_{n}\right) u_{n}\right\| \\
& +\sigma_{n+1}\left\|Q x_{n+1}-u_{n+1}\right\|+\sigma_{n}\left\|Q x_{n}-u_{n}\right\| \\
& \leq\left|\tau_{n+1}-\tau_{n}\right|\left\|\bar{u}_{n+1}\right\|+\tau_{n}\left\|\bar{u}_{n+1}-\bar{u}_{n}\right\| \\
& +\left|\tau_{n+1}-\tau_{n}\right|\left\|u_{n+1}\right\|+\left(1-\tau_{n}\right)\left\|u_{n+1}-u_{n}\right\| \\
& +\sigma_{n+1}\left\|Q x_{n+1}-u_{n+1}\right\|+\sigma_{n}\left\|Q x_{n}-u_{n}\right\| \\
& \leq \tau_{n}\left[\left\|x_{n+1}-x_{n}\right\|+\left\|z_{n+1}-z_{n}\right\|+\left|\lambda_{n+1} \alpha_{n+1}-\lambda_{n} \alpha_{n}\right|\left\|z_{n}\right\|\right. \\
& \left.+\left|\lambda_{n+1}-\lambda_{n}\right|\left\|\nabla f\left(z_{n}\right)\right\|\right]+\left(1-\tau_{n}\right)\left\|z_{n+1}-z_{n}\right\| \\
& +\left|\tau_{n+1}-\tau_{n}\right|\left(\left\|\bar{u}_{n+1}\right\|+\left\|u_{n+1}\right\|\right) \\
& +\sigma_{n+1}\left\|Q x_{n+1}-u_{n+1}\right\|+\sigma_{n}\left\|Q x_{n}-u_{n}\right\| \\
& \leq\left\|x_{n+1}-x_{n}\right\|+\left\|z_{n+1}-z_{n}\right\| \\
& +\left|\lambda_{n+1} \alpha_{n+1}-\lambda_{n} \alpha_{n}\right|\left\|z_{n}\right\| \\
& +\left|\lambda_{n+1}-\lambda_{n}\right|\left\|\nabla f\left(z_{n}\right)\right\|+\left|\tau_{n+1}-\tau_{n}\right|\left(\left\|\bar{u}_{n+1}\right\|+\left\|u_{n+1}\right\|\right) \\
& +\sigma_{n+1}\left\|Q x_{n+1}-u_{n+1}\right\|+\sigma_{n}\left\|Q x_{n}-u_{n}\right\| \text {. }
\end{aligned}
$$

Hence it follows from (113), and (117) that

$$
\begin{aligned}
& \left\|w_{n+1}-w_{n}\right\| \\
& \quad \leq \frac{\left\|\gamma_{n+1}\left(y_{n+1}-y_{n}\right)+\delta_{n+1}\left(S y_{n+1}-S y_{n}\right)\right\|}{1-\beta_{n+1}}
\end{aligned}
$$




$$
\begin{aligned}
& +\left|\frac{\gamma_{n+1}}{1-\beta_{n+1}}-\frac{\gamma_{n}}{1-\beta_{n}}\right|\left\|y_{n}\right\| \\
& +\left|\frac{\delta_{n+1}}{1-\beta_{n+1}}-\frac{\delta_{n}}{1-\beta_{n}}\right|\left\|S y_{n}\right\| \\
\leq & \frac{\gamma_{n+1}+\delta_{n+1}}{1-\beta_{n+1}}\left\|y_{n+1}-y_{n}\right\| \\
& +\left|\frac{\gamma_{n+1}}{1-\beta_{n+1}}-\frac{\gamma_{n}}{1-\beta_{n}}\right|\left(\left\|y_{n}\right\|+\left\|S y_{n}\right\|\right) \\
= & \left\|y_{n+1}-y_{n}\right\|+\left|\frac{\gamma_{n+1}}{1-\beta_{n+1}}-\frac{\gamma_{n}}{1-\beta_{n}}\right|\left(\left\|y_{n}\right\|+\left\|S y_{n}\right\|\right) \\
\leq & \left\|x_{n+1}-x_{n}\right\|+\left\|z_{n+1}-z_{n}\right\| \\
& +\left|\lambda_{n+1} \alpha_{n+1}-\lambda_{n} \alpha_{n}\right|\left\|z_{n}\right\|+\left|\lambda_{n+1}-\lambda_{n}\right|\left\|\nabla f\left(z_{n}\right)\right\| \\
& +\left|\tau_{n+1}-\tau_{n}\right|\left(\left\|\bar{u}_{n+1}\right\|+\left\|u_{n+1}\right\|\right) \\
& +\sigma_{n+1}\left\|Q x_{n+1}-u_{n+1}\right\|+\sigma_{n}\left\|Q x_{n}-u_{n}\right\| \\
& +\left|\frac{\gamma_{n+1}}{1-\beta_{n+1}}-\frac{\gamma_{n}}{1-\beta_{n}}\right|\left(\left\|y_{n}\right\|+\left\|S y_{n}\right\|\right) .
\end{aligned}
$$

Since $\left\{x_{n}\right\},\left\{y_{n}\right\},\left\{z_{n}\right\},\left\{u_{n}\right\}$, and $\left\{\bar{u}_{n}\right\}$ are bounded, it follows from conditions (i), (iii), (iv), (vi), and (vii) that

$$
\begin{aligned}
& \limsup _{n \rightarrow \infty}\left(\left\|w_{n+1}-w_{n}\right\|-\left\|x_{n+1}-x_{n}\right\|\right) \\
& \leq \limsup _{n \rightarrow \infty}\{\left\{\left|z_{n+1}-z_{n}\left\|+\left|\lambda_{n+1} \alpha_{n+1}-\lambda_{n} \alpha_{n}\right|\right\| z_{n} \|\right.\right. \\
&+\left|\lambda_{n+1}-\lambda_{n}\right|\left\|\nabla f\left(z_{n}\right)\right\| \\
&+\left|\tau_{n+1}-\tau_{n}\right|\left(\left\|\bar{u}_{n+1}\right\|+\left\|u_{n+1}\right\|\right) \\
&+ \sigma_{n+1}\left\|Q x_{n+1}-u_{n+1}\right\|+\sigma_{n}\left\|Q x_{n}-u_{n}\right\| \\
&\left.+\left|\frac{\gamma_{n+1}}{1-\beta_{n+1}}-\frac{\gamma_{n}}{1-\beta_{n}}\right|\left(\left\|y_{n}\right\|+\left\|S y_{n}\right\|\right)\right\}=0 .
\end{aligned}
$$

Hence by Lemma 14 we get $\lim _{n \rightarrow \infty}\left\|w_{n}-x_{n}\right\|=0$. Thus,

$$
\lim _{n \rightarrow \infty}\left\|x_{n+1}-x_{n}\right\|=\lim _{n \rightarrow \infty}\left(1-\beta_{n}\right)\left\|w_{n}-x_{n}\right\|=0 .
$$

Step 3. $\lim _{n \rightarrow \infty}\left\|B_{2} z_{n}-B_{2} p\right\|=0, \lim _{n \rightarrow \infty}\left\|B_{1} \widetilde{z}_{n}-B_{1} q\right\|=0$, and $\lim _{n \rightarrow \infty}\left\|x_{n}-z_{n}\right\|=0$, where $q=P_{C}\left(p-\mu_{2} B_{2} p\right)$.

Indeed, utilizing Lemma 17 and the convexity of $\|\cdot\|^{2}$, we obtain from (18) and (106)-(109) that

$$
\begin{aligned}
& \left\|x_{n+1}-p\right\|^{2} \\
& =\left\|\beta_{n}\left(x_{n}-p\right)+\gamma_{n}\left(y_{n}-p\right)+\delta_{n}\left(S y_{n}-p\right)\right\|^{2} \\
& \leq \beta_{n}\left\|x_{n}-p\right\|^{2}+\left(\gamma_{n}+\delta_{n}\right) \\
& \quad \times\left\|\frac{1}{\gamma_{n}+\delta_{n}}\left[\gamma_{n}\left(y_{n}-p\right)+\delta_{n}\left(S y_{n}-p\right)\right]\right\|^{2}
\end{aligned}
$$

$$
\begin{aligned}
& \leq \beta_{n}\left\|x_{n}-p\right\|^{2}+\left(\gamma_{n}+\delta_{n}\right)\left\|y_{n}-p\right\|^{2} \\
& \leq \beta_{n}\left\|x_{n}-p\right\|^{2}+\left(\gamma_{n}+\delta_{n}\right) \\
& \times\left[\sigma_{n}\left\|Q x_{n}-p\right\|^{2}+\tau_{n}\left\|\bar{u}_{n}-p\right\|^{2}+\left(1-\sigma_{n}-\tau_{n}\right)\left\|u_{n}-p\right\|^{2}\right] \\
& \leq \beta_{n}\left\|x_{n}-p\right\|^{2}+\sigma_{n}\left\|Q x_{n}-p\right\|^{2} \\
& +\left(\gamma_{n}+\delta_{n}\right)\left[\tau_{n}\left\|\bar{u}_{n}-p\right\|^{2}+\left(1-\tau_{n}\right)\left\|u_{n}-p\right\|^{2}\right] \\
& \leq \beta_{n}\left\|x_{n}-p\right\|^{2}+\sigma_{n}\left\|Q x_{n}-p\right\|^{2}+\left(\gamma_{n}+\delta_{n}\right) \\
& \times\left\{\tau _ { n } \left[\left\|x_{n}-p\right\|^{2}+2 \lambda_{n} \alpha_{n}\|p\|\left\|z_{n}-p\right\|\right.\right. \\
& \left.+\left(\lambda_{n}^{2}\left(\alpha_{n}+\|A\|^{2}\right)^{2}-1\right)\left\|x_{n}-z_{n}\right\|^{2}\right] \\
& +\left(1-\tau_{n}\right)\left[\left\|z_{n}-p\right\|^{2}-\mu_{2}\left(2 \beta_{2}-\mu_{2}\right)\left\|B_{2} z_{n}-B_{2} p\right\|^{2}\right. \\
& \left.\left.-\mu_{1}\left(2 \beta_{1}-\mu_{1}\right)\left\|B_{1} \widetilde{z}_{n}-B_{1} q\right\|^{2}\right]\right\} \\
& \leq \beta_{n}\left\|x_{n}-p\right\|^{2}+\sigma_{n}\left\|Q x_{n}-p\right\|^{2}+\left(\gamma_{n}+\delta_{n}\right) \\
& \times\left\{\tau _ { n } \left[\left\|x_{n}-p\right\|^{2}+2 \lambda_{n} \alpha_{n}\|p\|\left\|z_{n}-p\right\|\right.\right. \\
& \left.+\left(\lambda_{n}^{2}\left(\alpha_{n}+\|A\|^{2}\right)^{2}-1\right)\left\|x_{n}-z_{n}\right\|^{2}\right] \\
& +\left(1-\tau_{n}\right)\left[\left\|x_{n}-p\right\|^{2}+2 \lambda_{n} \alpha_{n}\|p\|\left\|z_{n}-p\right\|\right. \\
& -\mu_{2}\left(2 \beta_{2}-\mu_{2}\right)\left\|B_{2} z_{n}-B_{2} p\right\|^{2} \\
& \left.\left.-\mu_{1}\left(2 \beta_{1}-\mu_{1}\right)\left\|B_{1} \widetilde{z}_{n}-B_{1} q\right\|^{2}\right]\right\} \\
& =\beta_{n}\left\|x_{n}-p\right\|^{2}+\sigma_{n}\left\|Q x_{n}-p\right\|^{2}+\left(\gamma_{n}+\delta_{n}\right) \\
& \times\left\{\left\|x_{n}-p\right\|^{2}+2 \lambda_{n} \alpha_{n}\|p\|\left\|z_{n}-p\right\|\right. \\
& -\tau_{n}\left(1-\lambda_{n}^{2}\left(\alpha_{n}+\|A\|^{2}\right)^{2}\right)\left\|x_{n}-z_{n}\right\|^{2}-\left(1-\tau_{n}\right) \\
& \times\left[\mu_{2}\left(2 \beta_{2}-\mu_{2}\right)\left\|B_{2} z_{n}-B_{2} p\right\|^{2}\right. \\
& \left.\left.+\mu_{1}\left(2 \beta_{1}-\mu_{1}\right)\left\|B_{1} \widetilde{z}_{n}-B_{1} q\right\|^{2}\right]\right\} \\
& \leq\left\|x_{n}-p\right\|^{2}+\sigma_{n}\left\|Q x_{n}-p\right\|^{2}+2 \lambda_{n} \alpha_{n}\|p\|\left\|z_{n}-p\right\| \\
& -\left(\gamma_{n}+\delta_{n}\right)\left\{\tau_{n}\left(1-\lambda_{n}^{2}\left(\alpha_{n}+\|A\|^{2}\right)^{2}\right)\left\|x_{n}-z_{n}\right\|^{2}\right. \\
& +\left(1-\tau_{n}\right)\left[\mu_{2}\left(2 \beta_{2}-\mu_{2}\right)\left\|B_{2} z_{n}-B_{2} p\right\|^{2}\right. \\
& \left.\left.+\mu_{1}\left(2 \beta_{1}-\mu_{1}\right)\left\|B_{1} \widetilde{z}_{n}-B_{1} q\right\|^{2}\right]\right\} .
\end{aligned}
$$

Therefore,

$$
\begin{aligned}
\left(\gamma_{n}+\delta_{n}\right)\left\{\tau_{n}\left(1-\lambda_{n}^{2}\left(\alpha_{n}+\|A\|^{2}\right)^{2}\right)\left\|x_{n}-z_{n}\right\|^{2}\right. \\
+\left(1-\tau_{n}\right)\left[\mu_{2}\left(2 \beta_{2}-\mu_{2}\right)\left\|B_{2} z_{n}-B_{2} p\right\|^{2}\right.
\end{aligned}
$$




$$
\begin{aligned}
& \left.\left.\qquad \mu_{1}\left(2 \beta_{1}-\mu_{1}\right)\left\|B_{1} \widetilde{z}_{n}-B_{1} q\right\|^{2}\right]\right\} \\
& \leq\left\|x_{n}-p\right\|^{2}-\left\|x_{n+1}-p\right\|^{2} \\
& +\sigma_{n}\left\|Q x_{n}-p\right\|^{2}+2 \lambda_{n} \alpha_{n}\|p\|\left\|z_{n}-p\right\| \\
& \leq\left(\left\|x_{n}-p\right\|+\left\|x_{n+1}-p\right\|\right)\left\|x_{n}-x_{n+1}\right\| \\
& +\sigma_{n}\left\|Q x_{n}-p\right\|^{2}+2 \lambda_{n} \alpha_{n}\|p\|\left\|z_{n}-p\right\| .
\end{aligned}
$$

Since $\alpha_{n} \rightarrow 0, \sigma_{n} \rightarrow 0,\left\|x_{n}-x_{n+1}\right\| \rightarrow 0, \liminf _{n \rightarrow \infty}\left(\gamma_{n}+\right.$ $\left.\delta_{n}\right)>0,\left\{\lambda_{n}\right\} \subset[a, b]$, and $0<\liminf _{n \rightarrow \infty} \tau_{n} \leq \lim \sup _{n \rightarrow \infty}$ $\tau_{n}<1$, it follows that

$$
\begin{gathered}
\lim _{n \rightarrow \infty}\left\|x_{n}-z_{n}\right\|=0, \quad \lim _{n \rightarrow \infty}\left\|B_{1} \widetilde{z}_{n}-B_{1} q\right\|=0, \\
\lim _{n \rightarrow \infty}\left\|B_{2} z_{n}-B_{2} p\right\|=0 .
\end{gathered}
$$

Step 4. $\lim _{n \rightarrow \infty}\left\|S y_{n}-y_{n}\right\|=0$.

Indeed, observe that

$$
\begin{aligned}
\left\|\bar{u}_{n}-z_{n}\right\| & =\left\|P_{C}\left(x_{n}-\lambda_{n} \nabla f_{\alpha_{n}}\left(z_{n}\right)\right)-P_{C}\left(x_{n}-\lambda_{n} \nabla f_{\alpha_{n}}\left(x_{n}\right)\right)\right\| \\
& \leq\left\|\left(x_{n}-\lambda_{n} \nabla f_{\alpha_{n}}\left(z_{n}\right)\right)-\left(x_{n}-\lambda_{n} \nabla f_{\alpha_{n}}\left(x_{n}\right)\right)\right\| \\
& =\lambda_{n}\left\|\nabla f_{\alpha_{n}}\left(z_{n}\right)-\nabla f_{\alpha_{n}}\left(x_{n}\right)\right\| \\
& \leq \lambda_{n}\left(\alpha_{n}+\|A\|^{2}\right)\left\|z_{n}-x_{n}\right\| .
\end{aligned}
$$

This together with $\left\|z_{n}-x_{n}\right\| \rightarrow 0$ implies that $\lim _{n \rightarrow \infty} \| \bar{u}_{n}-$ $z_{n} \|=0$ and hence $\lim _{n \rightarrow \infty}\left\|\bar{u}_{n}-x_{n}\right\|=0$. Utilizing the arguments similar to those of (73) and (75) in the proof of Theorem 19 we can prove that

$$
\begin{aligned}
\left\|\tilde{z}_{n}-q\right\|^{2} \leq & \left\|z_{n}-p\right\|^{2}-\left\|z_{n}-\widetilde{z}_{n}-(p-q)\right\|^{2} \\
& +2 \mu_{2}\left\|z_{n}-\widetilde{z}_{n}-(p-q)\right\|\left\|B_{2} z_{n}-B_{2} p\right\|, \\
\left\|u_{n}-p\right\|^{2} \leq & \left\|\tilde{z}_{n}-q\right\|^{2}-\left\|\tilde{z}_{n}-u_{n}+(p-q)\right\|^{2} \\
& +2 \mu_{1}\left\|\widetilde{z}_{n}-u_{n}+(p-q)\right\|\left\|B_{1} \widetilde{z}_{n}-B_{1} q\right\| .
\end{aligned}
$$

Utilizing (106), (109), (125), we have

$$
\begin{aligned}
& \left\|y_{n}-p\right\|^{2} \\
& \quad=\left\|\sigma_{n}\left(Q x_{n}-p\right)+\tau_{n}\left(\bar{u}_{n}-p\right)+\left(1-\sigma_{n}-\tau_{n}\right)\left(u_{n}-p\right)\right\|^{2} \\
& \quad \leq \sigma_{n}\left\|Q x_{n}-p\right\|^{2}+\tau_{n}\left\|\bar{u}_{n}-p\right\|^{2}+\left(1-\sigma_{n}-\tau_{n}\right)\left\|u_{n}-p\right\|^{2} \\
& \quad \leq \sigma_{n}\left\|Q x_{n}-p\right\|^{2}+\tau_{n}\left\|\bar{u}_{n}-p\right\|^{2}
\end{aligned}
$$

$$
\begin{aligned}
& +\left(1-\tau_{n}\right)\left\|u_{n}-p\right\|^{2} \\
& \leq \sigma_{n}\left\|Q x_{n}-p\right\|^{2} \\
& +\tau_{n}\left(\left\|x_{n}-p\right\|^{2}+2 \lambda_{n} \alpha_{n}\|p\|\left\|z_{n}-p\right\|\right)+\left(1-\tau_{n}\right) \\
& \times\left[\left\|\widetilde{z}_{n}-q\right\|^{2}-\left\|\widetilde{z}_{n}-u_{n}+(p-q)\right\|^{2}\right. \\
& \left.+2 \mu_{1}\left\|\widetilde{z}_{n}-u_{n}+(p-q)\right\|\left\|B_{1} \widetilde{z}_{n}-B_{1} q\right\|\right] \\
& \leq \sigma_{n}\left\|Q x_{n}-p\right\|^{2} \\
& +\tau_{n}\left(\left\|x_{n}-p\right\|^{2}+2 \lambda_{n} \alpha_{n}\|p\|\left\|z_{n}-p\right\|\right)+\left(1-\tau_{n}\right) \\
& \times\left\{\left\|z_{n}-p\right\|^{2}-\left\|z_{n}-\widetilde{z}_{n}-(p-q)\right\|^{2}\right. \\
& +2 \mu_{2}\left\|z_{n}-\widetilde{z}_{n}-(p-q)\right\|\left\|B_{2} z_{n}-B_{2} p\right\| \\
& -\left\|\tilde{z}_{n}-u_{n}+(p-q)\right\|^{2} \\
& \left.+2 \mu_{1}\left\|\widetilde{z}_{n}-u_{n}+(p-q)\right\|\left\|B_{1} \widetilde{z}_{n}-B_{1} q\right\|\right\} \\
& \leq \sigma_{n}\left\|Q x_{n}-p\right\|^{2} \\
& +\tau_{n}\left(\left\|x_{n}-p\right\|^{2}+2 \lambda_{n} \alpha_{n}\|p\|\left\|z_{n}-p\right\|\right)+\left(1-\tau_{n}\right) \\
& \times\left\{\left\|x_{n}-p\right\|^{2}+2 \lambda_{n} \alpha_{n}\|p\|\left\|z_{n}-p\right\|\right. \\
& -\left\|z_{n}-\widetilde{z}_{n}-(p-q)\right\|^{2} \\
& +2 \mu_{2}\left\|z_{n}-\widetilde{z}_{n}-(p-q)\right\|\left\|B_{2} z_{n}-B_{2} p\right\| \\
& \text { - }\left\|\tilde{z}_{n}-u_{n}+(p-q)\right\|^{2} \\
& \left.+2 \mu_{1}\left\|\tilde{z}_{n}-u_{n}+(p-q)\right\|\left\|B_{1} \widetilde{z}_{n}-B_{1} q\right\|\right\} \\
& \leq\left\|x_{n}-p\right\|^{2}+\sigma_{n}\left\|Q x_{n}-p\right\|^{2}+2 \lambda_{n} \alpha_{n}\|p\|\left\|z_{n}-p\right\| \\
& +2 \mu_{2}\left\|z_{n}-\widetilde{z}_{n}-(p-q)\right\|\left\|B_{2} z_{n}-B_{2} p\right\| \\
& +2 \mu_{1}\left\|\widetilde{z}_{n}-u_{n}+(p-q)\right\|\left\|B_{1} \widetilde{z}_{n}-B_{1} q\right\| \\
& -\left(1-\tau_{n}\right)\left(\left\|z_{n}-\widetilde{z}_{n}-(p-q)\right\|^{2}+\left\|\widetilde{z}_{n}-u_{n}+(p-q)\right\|^{2}\right) \text {. }
\end{aligned}
$$

Thus from (18) and (126) it follows that

$$
\begin{aligned}
&\left\|x_{n+1}-p\right\|^{2} \\
&=\left\|\beta_{n}\left(x_{n}-p\right)+\gamma_{n}\left(y_{n}-p\right)+\delta_{n}\left(S y_{n}-p\right)\right\|^{2} \\
& \leq \beta_{n}\left\|x_{n}-p\right\|^{2}+\left(\gamma_{n}+\delta_{n}\right)\left\|y_{n}-p\right\|^{2} \\
&= \beta_{n}\left\|x_{n}-p\right\|^{2}+\left(1-\beta_{n}\right)\left\|y_{n}-p\right\|^{2} \\
& \leq \beta_{n}\left\|x_{n}-p\right\|^{2}+\left(1-\beta_{n}\right) \\
&\left\{\left\|x_{n}-p\right\|^{2}+\sigma_{n}\left\|Q x_{n}-p\right\|^{2}\right. \\
& \quad+2 \lambda_{n} \alpha_{n}\|p\|\left\|z_{n}-p\right\|
\end{aligned}
$$




$$
\begin{gathered}
+2 \mu_{2}\left\|z_{n}-\widetilde{z}_{n}-(p-q)\right\|\left\|B_{2} z_{n}-B_{2} p\right\| \\
+2 \mu_{1}\left\|\widetilde{z}_{n}-u_{n}+(p-q)\right\|\left\|B_{1} \widetilde{z}_{n}-B_{1} q\right\| \\
-\left(1-\tau_{n}\right)\left(\left\|z_{n}-\widetilde{z}_{n}-(p-q)\right\|^{2}\right. \\
\left.\left.+\left\|\widetilde{z}_{n}-u_{n}+(p-q)\right\|^{2}\right)\right\} \\
\leq\left\|x_{n}-p\right\|^{2}+\sigma_{n}\left\|Q x_{n}-p\right\|^{2}+2 \lambda_{n} \alpha_{n}\|p\|\left\|z_{n}-p\right\| \\
+2 \mu_{2}\left\|z_{n}-\widetilde{z}_{n}-(p-q)\right\|\left\|B_{2} z_{n}-B_{2} p\right\| \\
+2 \mu_{1}\left\|\tilde{z}_{n}-u_{n}+(p-q)\right\|\left\|B_{1} \widetilde{z}_{n}-B_{1} q\right\| \\
-\left(1-\beta_{n}\right)\left(1-\tau_{n}\right) \\
\times\left(\left\|z_{n}-\widetilde{z}_{n}-(p-q)\right\|^{2}+\left\|\widetilde{z}_{n}-u_{n}+(p-q)\right\|^{2}\right),
\end{gathered}
$$

which hence implies that

$$
\begin{aligned}
& \left(1-\beta_{n}\right)\left(1-\tau_{n}\right) \\
& \times\left(\left\|z_{n}-\widetilde{z}_{n}-(p-q)\right\|^{2}+\left\|\widetilde{z}_{n}-u_{n}+(p-q)\right\|^{2}\right) \\
& \leq\left\|x_{n}-p\right\|^{2}-\left\|x_{n+1}-p\right\|^{2} \\
& +\sigma_{n}\left\|\mathrm{Q} x_{n}-p\right\|^{2}+2 \lambda_{n} \alpha_{n}\|p\|\left\|z_{n}-p\right\| \\
& +2 \mu_{2}\left\|z_{n}-\widetilde{z}_{n}-(p-q)\right\|\left\|B_{2} z_{n}-B_{2} p\right\| \\
& +2 \mu_{1}\left\|\widetilde{z}_{n}-u_{n}+(p-q)\right\|\left\|B_{1} \widetilde{z}_{n}-B_{1} q\right\| \\
& \leq\left(\left\|x_{n}-p\right\|+\left\|x_{n+1}-p\right\|\right)\left\|x_{n}-x_{n+1}\right\| \\
& +\sigma_{n}\left\|Q x_{n}-p\right\|^{2}+2 \lambda_{n} \alpha_{n}\|p\|\left\|z_{n}-p\right\| \\
& +2 \mu_{2}\left\|z_{n}-\widetilde{z}_{n}-(p-q)\right\|\left\|B_{2} z_{n}-B_{2} p\right\| \\
& +2 \mu_{1}\left\|\tilde{z}_{n}-u_{n}+(p-q)\right\|\left\|B_{1} \widetilde{z}_{n}-B_{1} q\right\| \text {. }
\end{aligned}
$$

Since $\lim \sup _{n \rightarrow \infty} \beta_{n}<1, \limsup _{n \rightarrow \infty} \tau_{n}<1,\left\{\lambda_{n}\right\} \quad C$ $[a, b], \alpha_{n} \rightarrow 0, \sigma_{n} \rightarrow 0,\left\|B_{2} z_{n}-B_{2} p\right\| \rightarrow 0, \| B_{1} \widetilde{z}_{n}-$ $B_{1} q \| \rightarrow 0$ and $\left\|x_{n+1}-x_{n}\right\| \rightarrow 0$, it follows from the boundedness of $\left\{x_{n}\right\},\left\{u_{n}\right\},\left\{z_{n}\right\}$, and $\left\{\widetilde{z}_{n}\right\}$ that

$$
\begin{aligned}
& \lim _{n \rightarrow \infty}\left\|z_{n}-\tilde{z}_{n}-(p-q)\right\|=0, \\
& \lim _{n \rightarrow \infty}\left\|\tilde{z}_{n}-u_{n}+(p-q)\right\|=0 .
\end{aligned}
$$

Consequently, it immediately follows that

$$
\lim _{n \rightarrow \infty}\left\|z_{n}-u_{n}\right\|=0, \quad \lim _{n \rightarrow \infty}\left\|u_{n}-\bar{u}_{n}\right\|=0 .
$$

Also, note that

$$
\left\|y_{n}-\bar{u}_{n}\right\| \leq \sigma_{n}\left\|Q x_{n}-\bar{u}_{n}\right\|+\left(1-\sigma_{n}-\tau_{n}\right)\left\|u_{n}-\bar{u}_{n}\right\| \longrightarrow 0 .
$$

This together with $\left\|x_{n}-\bar{u}_{n}\right\| \rightarrow 0$ implies that

$$
\lim _{n \rightarrow \infty}\left\|x_{n}-y_{n}\right\|=0 \text {. }
$$

Since

$$
\left\|\delta_{n}\left(S y_{n}-x_{n}\right)\right\| \leq\left\|x_{n+1}-x_{n}\right\|+\gamma_{n}\left\|y_{n}-x_{n}\right\|,
$$

it follows that

$$
\lim _{n \rightarrow \infty}\left\|S y_{n}-x_{n}\right\|=0, \quad \lim _{n \rightarrow \infty}\left\|S y_{n}-y_{n}\right\|=0 .
$$

Step 5. $\lim \sup _{n \rightarrow \infty}\left\langle Q \bar{x}-\bar{x}, x_{n}-\bar{x}\right\rangle \leq 0$ where $\bar{x}=$ $P_{\mathrm{Fix}(S) \cap \Xi \cap \Gamma} Q \bar{x}$.

Indeed, since $\left\{x_{n}\right\}$ is bounded, there exists a subsequence $\left\{x_{n_{i}}\right\}$ of $\left\{x_{n}\right\}$ such that

$$
\limsup _{n \rightarrow \infty}\left\langle Q \bar{x}-\bar{x}, x_{n}-\bar{x}\right\rangle=\lim _{i \rightarrow \infty}\left\langle Q \bar{x}-\bar{x}, x_{n_{i}}-\bar{x}\right\rangle .
$$

Also, since $H$ is reflexive and $\left\{x_{n}\right\}$ is bounded, without loss of generality we may assume that $x_{n_{i}} \rightarrow \widehat{p}$ weakly for some $\widehat{p} \in$ $C$. Taking into account that $\left\|x_{n}-y_{n}\right\| \rightarrow 0$ and $\left\|x_{n}-z_{n}\right\| \rightarrow 0$ as $n \rightarrow \infty$, we deduce that $y_{n_{i}} \rightarrow \widehat{p}$ weakly and $z_{n_{i}} \rightarrow \widehat{p}$ weakly.

First, it is clear from Lemma 15 and $\left\|S y_{n}-y_{n}\right\| \rightarrow 0$ that $\widehat{p} \in \operatorname{Fix}(S)$. Now let us show that $\widehat{p} \in \Xi$. Note that

$$
\begin{aligned}
& \left\|z_{n}-G\left(z_{n}\right)\right\| \\
& \quad=\left\|z_{n}-P_{C}\left[P_{C}\left(z_{n}-\mu_{2} B_{2} z_{n}\right)-\mu_{1} B_{1} P_{C}\left(z_{n}-\mu_{2} B_{2} z_{n}\right)\right]\right\| \\
& \quad=\left\|z_{n}-u_{n}\right\| \longrightarrow 0 \quad(n \longrightarrow \infty),
\end{aligned}
$$

where $G: C \rightarrow C$ is defined as that in Lemma 4. According to Lemma 15 we get $\hat{p} \in \Xi$. Further, let us show that $\hat{p} \in \Gamma$. As a matter of fact, define

$$
T v=\left\{\begin{array}{cl}
\nabla f(v)+N_{C} v, & \text { if } v \in C, \\
\emptyset, & \text { if } v \notin C,
\end{array}\right.
$$

where $N_{C} v=\left\{w \in \mathscr{H}_{1}:\langle v-u, w\rangle \geq 0, \forall u \in C\right\}$. Then, $T$ is maximal monotone and $0 \in T v$ if and only if $v \in \mathrm{VI}(C, \nabla f)$; see [40] for more details. Let $(v, w) \in \operatorname{Gph}(T)$. Then, we have

$$
\langle v-u, w-\nabla f(v)\rangle \geq 0, \quad \forall u \in C .
$$

Observe that

$$
z_{n}=P_{C}\left(x_{n}-\lambda_{n} \nabla f_{\alpha_{n}}\left(x_{n}\right)\right), \quad v \in C .
$$

Utilizing the arguments similar to those of Step 5 in the proof of Theorem 19 we can prove that

$$
\langle v-\widehat{p}, w\rangle \geq 0 .
$$

Since $T$ is maximal monotone, we have $\widehat{p} \in T^{-1} 0$, and, hence, $\hat{p} \in \operatorname{VI}(C, \nabla f)$. Thus it is clear that $\widehat{p} \in \Gamma$. Therefore, $\widehat{p} \in$ $\operatorname{Fix}(S) \cap \Xi \cap \Gamma$. Consequently, in terms of Proposition 8 (i) we obtain from (135) that

$$
\begin{aligned}
\limsup _{n \rightarrow \infty}\left\langle Q \bar{x}-\bar{x}, x_{n}-\bar{x}\right\rangle & =\lim _{i \rightarrow \infty}\left\langle Q \bar{x}-\bar{x}, x_{n_{i}}-\bar{x}\right\rangle \\
& =\langle Q \bar{x}-\bar{x}, \bar{p}-\bar{x}\rangle \leq 0 .
\end{aligned}
$$


Step 6. $\lim _{n \rightarrow \infty}\left\|x_{n}-\bar{x}\right\|=0$.

Indeed, observe that

$$
\begin{aligned}
\left\langle Q x_{n}-\bar{x}, y_{n}-\bar{x}\right\rangle & \\
= & \left\langle Q x_{n}-\bar{x}, x_{n}-\bar{x}\right\rangle+\left\langle Q x_{n}-\bar{x}, y_{n}-x_{n}\right\rangle \\
= & \left\langle Q x_{n}-Q \bar{x}, x_{n}-\bar{x}\right\rangle \\
& +\left\langle Q \bar{x}-\bar{x}, x_{n}-\bar{x}\right\rangle+\left\langle Q x_{n}-\bar{x}, y_{n}-x_{n}\right\rangle \\
\leq & \rho\left\|x_{n}-\bar{x}\right\|^{2}+\left\langle Q \bar{x}-\bar{x}, x_{n}-\bar{x}\right\rangle \\
& +\left\|Q x_{n}-\bar{x}\right\|\left\|y_{n}-x_{n}\right\| .
\end{aligned}
$$

Utilizing Lemmas 17 and 18, we obtain from (106)-(109) and the convexity of $\|\cdot\|^{2}$ that

$$
\begin{aligned}
& \left\|x_{n+1}-\bar{x}\right\|^{2} \\
& =\left\|\beta_{n}\left(x_{n}-\bar{x}\right)+\gamma_{n}\left(y_{n}-\bar{x}\right)+\delta_{n}\left(S y_{n}-\bar{x}\right)\right\|^{2} \\
& \leq \beta_{n}\left\|x_{n}-\bar{x}\right\|^{2} \\
& +\left(\gamma_{n}+\delta_{n}\right)\left\|\frac{1}{\gamma_{n}+\delta_{n}}\left[\gamma_{n}\left(y_{n}-\bar{x}\right)+\delta_{n}\left(S y_{n}-\bar{x}\right)\right]\right\|^{2} \\
& \leq \beta_{n}\left\|x_{n}-\bar{x}\right\|^{2}+\left(\gamma_{n}+\delta_{n}\right)\left\|y_{n}-\bar{x}\right\|^{2} \\
& \leq \beta_{n}\left\|x_{n}-\bar{x}\right\|^{2}+\left(\gamma_{n}+\delta_{n}\right) \\
& \times\left[\left\|\tau_{n}\left(\bar{u}_{n}-\bar{x}\right)+\left(1-\sigma_{n}-\tau_{n}\right)\left(u_{n}-\bar{x}\right)\right\|^{2}\right. \\
& \left.+2 \sigma_{n}\left\langle Q x_{n}-\bar{x}, y_{n}-\bar{x}\right\rangle\right] \\
& \leq \beta_{n}\left\|x_{n}-\bar{x}\right\|^{2}+\left(\gamma_{n}+\delta_{n}\right) \\
& \times\left[\tau_{n}\left\|\bar{u}_{n}-\bar{x}\right\|^{2}+\left(1-\sigma_{n}-\tau_{n}\right)\left\|u_{n}-\bar{x}\right\|^{2}\right. \\
& \left.+2 \sigma_{n}\left\langle Q x_{n}-\bar{x}, y_{n}-\bar{x}\right\rangle\right] \\
& \leq \beta_{n}\left\|x_{n}-\bar{x}\right\|^{2}+\left(\gamma_{n}+\delta_{n}\right) \\
& \times\left[\tau_{n}\left\|\bar{u}_{n}-\bar{x}\right\|^{2}+\left(1-\sigma_{n}-\tau_{n}\right)\left\|z_{n}-\bar{x}\right\|^{2}\right. \\
& \left.+2 \sigma_{n}\left\langle Q x_{n}-\bar{x}, y_{n}-\bar{x}\right\rangle\right] \\
& \leq \beta_{n}\left\|x_{n}-\bar{x}\right\|^{2}+\left(\gamma_{n}+\delta_{n}\right) \\
& \times\left[\tau_{n}\left(\left\|x_{n}-\bar{x}\right\|^{2}+2 \lambda_{n} \alpha_{n}\|\bar{x}\|\left\|z_{n}-\bar{x}\right\|\right)\right. \\
& +\left(1-\sigma_{n}-\tau_{n}\right) \\
& \times\left(\left\|x_{n}-\bar{x}\right\|^{2}+2 \lambda_{n} \alpha_{n}\|\bar{x}\|\left\|z_{n}-\bar{x}\right\|\right) \\
& \left.+2 \sigma_{n}\left\langle Q x_{n}-\bar{x}, y_{n}-\bar{x}\right\rangle\right] \\
& =\beta_{n}\left\|x_{n}-\bar{x}\right\|^{2}+\left(\gamma_{n}+\delta_{n}\right) \\
& \times\left[\left(1-\sigma_{n}\right)\left(\left\|x_{n}-\bar{x}\right\|^{2}+2 \lambda_{n} \alpha_{n}\|\bar{x}\|\left\|z_{n}-\bar{x}\right\|\right)\right.
\end{aligned}
$$

$$
\begin{aligned}
& \left.+2 \sigma_{n}\left\langle Q x_{n}-\bar{x}, y_{n}-\bar{x}\right\rangle\right] \\
= & \left(1-\left(\gamma_{n}+\delta_{n}\right) \sigma_{n}\right)\left\|x_{n}-\bar{x}\right\|^{2} \\
+ & \left(\gamma_{n}+\delta_{n}\right)\left(1-\sigma_{n}\right) 2 \lambda_{n} \alpha_{n}\|\bar{x}\|\left\|z_{n}-\bar{x}\right\| \\
+ & \left(\gamma_{n}+\delta_{n}\right) 2 \sigma_{n}\left\langle Q x_{n}-\bar{x}, y_{n}-\bar{x}\right\rangle \\
\leq & \left(1-\left(\gamma_{n}+\delta_{n}\right) \sigma_{n}\right)\left\|x_{n}-\bar{x}\right\|^{2}+\left(\gamma_{n}+\delta_{n}\right) 2 \sigma_{n} \\
& \times\left[\rho\left\|x_{n}-\bar{x}\right\|^{2}\right. \\
& \left.+\left\langle Q \bar{x}-\bar{x}, x_{n}-\bar{x}\right\rangle+\left\|Q x_{n}-\bar{x}\right\|\left\|y_{n}-x_{n}\right\|\right] \\
+ & 2 \lambda_{n} \alpha_{n}\|\bar{x}\|\left\|z_{n}-\bar{x}\right\| \\
= & {\left[1-(1-2 \rho)\left(\gamma_{n}+\delta_{n}\right) \sigma_{n}\right]\left\|x_{n}-\bar{x}\right\|^{2} } \\
& +(1-2 \rho)\left(\gamma_{n}+\delta_{n}\right) \sigma_{n} \\
& \times \frac{2\left[\left\langle Q \bar{x}-\bar{x}, x_{n}-\bar{x}\right\rangle+\left\|Q x_{n}-\bar{x}\right\|\left\|y_{n}-x_{n}\right\|\right]}{1-2 \rho} \\
+ & 2 \lambda_{n} \alpha_{n}\|\bar{x}\|\left\|z_{n}-\bar{x}\right\| .
\end{aligned}
$$

Note that $\liminf _{n \rightarrow \infty}(1-2 \rho)\left(\gamma_{n}+\delta_{n}\right)>0$. It follows that $\sum_{n=0}^{\infty}(1-2 \rho)\left(\gamma_{n}+\delta_{n}\right) \sigma_{n}=\infty$. It is clear that

$$
\limsup _{n \rightarrow \infty} \frac{2\left[\left\langle Q \bar{x}-\bar{x}, x_{n}-\bar{x}\right\rangle+\left\|Q x_{n}-\bar{x}\right\|\left\|y_{n}-x_{n}\right\|\right]}{1-2 \rho} \leq 0 \text {, }
$$

because $\limsup _{n \rightarrow \infty}\left\langle Q \bar{x}-\bar{x}, x_{n}-\bar{x}\right\rangle \leq 0$ and $\lim _{n \rightarrow \infty} \| x_{n}-$ $y_{n} \|=0$. In addition, note also that $\left\{\lambda_{n}\right\} \subset[a, b], \sum_{n=0}^{\infty} \alpha_{n}<$ $\infty$ and $\left\{z_{n}\right\}$ is bounded. Hence we get $\sum_{n=0}^{\infty} 2 \lambda_{n} \alpha_{n}\|\bar{x}\| \| z_{n}-$ $\bar{x} \|<\infty$. Therefore, all conditions of Lemma 16 are satisfied. Consequently, we immediately deduce that $\left\|x_{n}-\bar{x}\right\| \rightarrow 0$ as $n \rightarrow \infty$. In the meantime, taking into account that $\| x_{n}-$ $y_{n} \| \rightarrow 0$ and $\left\|x_{n}-z_{n}\right\| \rightarrow 0$ as $n \rightarrow \infty$, we infer that

$$
\lim _{n \rightarrow \infty}\left\|y_{n}-\bar{x}\right\|=\lim _{n \rightarrow \infty}\left\|z_{n}-\bar{x}\right\|=0 .
$$

This completes the proof.

Corollary 23. Let $C$ be a nonempty closed convex subset of a real Hilbert space $\mathscr{H}_{1}$. Let $A \in B\left(\mathscr{H}_{1}, \mathscr{H}_{2}\right)$ and let $B_{i}: C \rightarrow$ $\mathscr{H}_{1}$ be $\beta_{i}$-inverse strongly monotone for $i=1,2$. Let $S: C \rightarrow C$ be a $k$-strictly pseudocontractive mapping such that $\operatorname{Fix}(S) \cap$ $\Xi \cap \Gamma \neq \emptyset$. For fixed $u \in C$ and given $x_{0} \in C$ arbitrarily, let the sequences $\left\{x_{n}\right\},\left\{y_{n}\right\},\left\{z_{n}\right\}$ be generated iteratively by

$$
\begin{aligned}
z_{n}= & P_{C}\left(x_{n}-\lambda_{n} \nabla f_{\alpha_{n}}\left(x_{n}\right)\right), \\
y_{n}= & \sigma_{n} u+\tau_{n} P_{C}\left(x_{n}-\lambda_{n} \nabla f_{\alpha_{n}}\left(z_{n}\right)\right) \\
& +\left(1-\sigma_{n}-\tau_{n}\right) \\
& \times P_{C}\left[P_{C}\left(z_{n}-\mu_{2} B_{2} z_{n}\right)-\mu_{1} B_{1} P_{C}\left(z_{n}-\mu_{2} B_{2} z_{n}\right)\right], \\
x_{n+1}= & \beta_{n} x_{n}+\gamma_{n} y_{n}+\delta_{n} S y_{n}, \quad \forall n \geq 0,
\end{aligned}
$$


where $\mu_{i} \in\left(0,2 \beta_{i}\right)$ for $i=1,2,\left\{\alpha_{n}\right\} \subset(0, \infty),\left\{\lambda_{n}\right\} \subset$ $\left(0,1 /\|A\|^{2}\right)$ and $\left\{\sigma_{n}\right\},\left\{\tau_{n}\right\},\left\{\beta_{n}\right\},\left\{\gamma_{n}\right\},\left\{\delta_{n}\right\} \subset[0,1]$ such that

(i) $\sum_{n=0}^{\infty} \alpha_{n}<\infty$;

(ii) $\sigma_{n}+\tau_{n} \leq 1, \beta_{n}+\gamma_{n}+\delta_{n}=1$ and $\left(\gamma_{n}+\delta_{n}\right) k \leq \gamma_{n}$ for all $n \geq 0$;

(iii) $\lim _{n \rightarrow \infty} \sigma_{n}=0$ and $\sum_{n=0}^{\infty} \sigma_{n}=\infty$;

(iv) $0<\liminf _{n \rightarrow \infty} \tau_{n} \leq \limsup _{n \rightarrow \infty} \tau_{n}<1$ and $\lim _{n \rightarrow \infty}\left|\tau_{n+1}-\tau_{n}\right|=0 ;$

(v) $0<\liminf _{n \rightarrow \infty} \beta_{n} \leq \limsup _{n \rightarrow \infty} \beta_{n}<1$ and $\liminf _{n \rightarrow \infty} \delta_{n}>0$;

(vi) $\lim _{n \rightarrow \infty}\left(\gamma_{n+1} /\left(1-\beta_{n+1}\right)-\gamma_{n} /\left(1-\beta_{n}\right)\right)=0$;

(vii) $0<\liminf _{n \rightarrow \infty} \lambda_{n} \leq \limsup _{n \rightarrow \infty} \lambda_{n}<1 /\|A\|^{2}$ and $\lim _{n \rightarrow \infty}\left|\lambda_{n+1}-\lambda_{n}\right|=0$.

Then the sequences $\left\{x_{n}\right\},\left\{y_{n}\right\},\left\{z_{n}\right\}$ converge strongly to the same point $\bar{x}=P_{\mathrm{Fix}(S) \cap \Xi \cap \Gamma} u$ if and only if $\lim _{n \rightarrow \infty}\left\|z_{n+1}-z_{n}\right\|=$ 0 . Furthermore, $(\bar{x}, \bar{y})$ is a solution of the GSVI (14), where $\bar{y}=P_{C}\left(\bar{x}-\mu_{2} B_{2} \bar{x}\right)$.

Corollary 24. Let $C$ be a nonempty closed convex subset of a real Hilbert space $\mathscr{H}_{1}$. Let $A \in B\left(\mathscr{H}_{1}, \mathscr{H}_{2}\right)$ and let $B_{i}: C \rightarrow$ $\mathscr{H}_{1}$ be $\beta_{i}$-inverse strongly monotone for $i=1,2$. Let $S: C \rightarrow$ $C$ be a nonexpansive mapping such that $\operatorname{Fix}(S) \cap \Xi \cap \Gamma \neq \emptyset$. Let $Q: C \rightarrow C$ be a $\rho$-contraction with $\rho \in[0,1 / 2)$. For given $x_{0} \in C$ arbitrarily, let the sequences $\left\{x_{n}\right\},\left\{y_{n}\right\},\left\{z_{n}\right\}$ be generated iteratively by

$$
\begin{aligned}
z_{n}= & P_{C}\left(x_{n}-\lambda_{n} \nabla f_{\alpha_{n}}\left(x_{n}\right)\right), \\
y_{n}= & \sigma_{n} Q x_{n}+\tau_{n} P_{C}\left(x_{n}-\lambda_{n} \nabla f_{\alpha_{n}}\left(z_{n}\right)\right) \\
& +\left(1-\sigma_{n}-\tau_{n}\right) \\
& \times P_{C}\left[P_{C}\left(z_{n}-\mu_{2} B_{2} z_{n}\right)-\mu_{1} B_{1} P_{C}\left(z_{n}-\mu_{2} B_{2} z_{n}\right)\right], \\
x_{n+1}= & \beta_{n} x_{n}+\gamma_{n} y_{n}+\delta_{n} S y_{n}, \quad \forall n \geq 0,
\end{aligned}
$$

where $\mu_{i} \in\left(0,2 \beta_{i}\right)$ for $i=1,2,\left\{\alpha_{n}\right\} \subset(0, \infty),\left\{\lambda_{n}\right\} \subset$ $\left(0,1 /\|A\|^{2}\right)$ and $\left\{\sigma_{n}\right\},\left\{\tau_{n}\right\},\left\{\beta_{n}\right\},\left\{\gamma_{n}\right\},\left\{\delta_{n}\right\} \subset[0,1]$ such that

(i) $\sum_{n=0}^{\infty} \alpha_{n}<\infty$;

(ii) $\sigma_{n}+\tau_{n} \leq 1, \beta_{n}+\gamma_{n}+\delta_{n}=1$ and $\left(\gamma_{n}+\delta_{n}\right) k \leq \gamma_{n}$ for all $n \geq 0$;

(iii) $\lim _{n \rightarrow \infty} \sigma_{n}=0$ and $\sum_{n=0}^{\infty} \sigma_{n}=\infty$;

(iv) $0<\liminf _{n \rightarrow \infty} \tau_{n} \leq \limsup _{n \rightarrow \infty} \tau_{n}<1$ and $\lim _{n \rightarrow \infty}\left|\tau_{n+1}-\tau_{n}\right|=0 ;$

(v) $0<\liminf _{n \rightarrow \infty} \beta_{n} \leq \limsup _{n \rightarrow \infty} \beta_{n}<1$ and $\liminf _{n \rightarrow \infty} \delta_{n}>0$;

(vi) $\lim _{n \rightarrow \infty}\left(\gamma_{n+1} /\left(1-\beta_{n+1}\right)-\gamma_{n} /\left(1-\beta_{n}\right)\right)=0$;

(vii) $0<\liminf _{n \rightarrow \infty} \lambda_{n} \leq \limsup _{n \rightarrow \infty} \lambda_{n}<1 /\|A\|^{2}$ and $\lim _{n \rightarrow \infty}\left|\lambda_{n+1}-\lambda_{n}\right|=0$.

Then the sequences $\left\{x_{n}\right\},\left\{y_{n}\right\},\left\{z_{n}\right\}$ converge strongly to the same point $\bar{x}=P_{\text {Fix }(S) \cap \Xi \cap \Gamma} Q \bar{x}$ if and only if $\lim _{n \rightarrow \infty} \| z_{n+1}-$ $z_{n} \|=0$. Furthermore, $(\bar{x}, \bar{y})$ is a solution of the GSVI (14), where $\bar{y}=P_{C}\left(\bar{x}-\mu_{2} B_{2} \bar{x}\right)$.
Next, utilizing Corollary 23 one gives the following improvement and extension of the main result in [18] (i.e., [18, Theorem 3.1]).

Corollary 25. Let $C$ be a nonempty closed convex subset of a real Hilbert space $\mathscr{H}_{1}$. Let $A \in B\left(\mathscr{H}_{1}, \mathscr{H}_{2}\right)$ and let $S: C \rightarrow C$ be a nonexpansive mapping such that $\operatorname{Fix}(S) \cap \Gamma \neq \emptyset$. For fixed $u \in C$ and given $x_{0} \in C$ arbitrarily, let the sequences $\left\{x_{n}\right\},\left\{z_{n}\right\}$ be generated iteratively by

$$
\begin{gathered}
z_{n}=P_{C}\left(x_{n}-\lambda_{n} \nabla f_{\alpha_{n}}\left(x_{n}\right)\right), \\
x_{n+1}=\beta_{n} x_{n}+\left(1-\beta_{n}\right) \\
\times S\left[\sigma_{n} u+\tau_{n} P_{C}\left(x_{n}-\lambda_{n} \nabla f_{\alpha_{n}}\left(z_{n}\right)\right)\right. \\
\left.\quad+\left(1-\sigma_{n}-\tau_{n}\right) z_{n}\right], \quad \forall n \geq 0,
\end{gathered}
$$

where $\left\{\alpha_{n}\right\} \subset(0, \infty),\left\{\lambda_{n}\right\} \subset\left(0,1 /\|A\|^{2}\right)$ and $\left\{\sigma_{n}\right\},\left\{\tau_{n}\right\},\left\{\beta_{n}\right\} \subset$ $[0,1]$ such that

(i) $\sum_{n=0}^{\infty} \alpha_{n}<\infty$;

(ii) $\sigma_{n}+\tau_{n} \leq 1$ for all $n \geq 0$;

(iii) $\lim _{n \rightarrow \infty} \sigma_{n}=0$ and $\sum_{n=0}^{\infty} \sigma_{n}=\infty$;

(iv) $0<\liminf _{n \rightarrow \infty} \tau_{n} \leq \limsup _{n \rightarrow \infty} \tau_{n}<1$ and $\lim _{n \rightarrow \infty}\left|\tau_{n+1}-\tau_{n}\right|=0$;

(v) $0<\liminf _{n \rightarrow \infty} \beta_{n} \leq \limsup _{n \rightarrow \infty} \beta_{n}<1$;

(vi) $0<\liminf _{n \rightarrow \infty} \lambda_{n} \leq \limsup _{n \rightarrow \infty} \lambda_{n}<1 /\|A\|^{2}$ and $\lim _{n \rightarrow \infty}\left|\lambda_{n+1}-\lambda_{n}\right|=0$.

Then the sequences $\left\{x_{n}\right\},\left\{z_{n}\right\}$ converge strongly to the same point $\bar{x}=P_{\mathrm{Fix}(S) \cap \Gamma} u$ if and only if $\lim _{n \rightarrow \infty}\left\|z_{n+1}-z_{n}\right\|=0$.

Proof. In Corollary 23, put $B_{1}=B_{2}=0$ and $\gamma_{n}=0$. Then, $\Xi=$ $C, \beta_{n}+\delta_{n}=1, P_{C}\left[P_{C}\left(z_{n}-\mu_{2} B_{2} z_{n}\right)-\mu_{1} B_{1} P_{C}\left(z_{n}-\mu_{2} B_{2} z_{n}\right)\right]=z_{n}$, and the iterative scheme (146) is equivalent to

$$
\begin{gathered}
z_{n}=P_{C}\left(x_{n}-\lambda_{n} \nabla f_{\alpha_{n}}\left(x_{n}\right)\right), \\
y_{n}=\sigma_{n} u+\tau_{n} P_{C}\left(x_{n}-\lambda_{n} \nabla f_{\alpha_{n}}\left(z_{n}\right)\right)+\left(1-\sigma_{n}-\tau_{n}\right) z_{n}, \\
x_{n+1}=\beta_{n} x_{n}+\delta_{n} S y_{n}, \quad \forall n \geq 0 .
\end{gathered}
$$

This is equivalent to (148). Since $S$ is a nonexpansive mapping, $S$ must be a $k$-strictly pseudocontractive mapping with $k=$ 0 . In this case, it is easy to see that conditions (i)-(vii) in Corollary 23 all are satisfied. Therefore, in terms of Corollary 23, we obtain the desired result.

Remark 26. Our Theorems 19 and 22 improve, extend, and develop [6, Theorem 5.7], [18, Theorem 3.1], and [33, Theorem 3.1] in the following aspects.

(i) Because both [6, Theorem 5.7] and [18, Theorem 3.1] are weak convergence results for solving the SFP, beyond question, our Theorems 19 and 22 as strong convergence results are very interesting and quite valuable. 
(ii) The problem of finding an element of $\operatorname{Fix}(S) \cap \Xi \cap \Gamma$ in our Theorems 19 and 22 is more general than the corresponding problems in [6, Theorem 5.7] and [18, Theorem 3.1], respectively.

(iii) The relaxed extragradient iterative method for finding an element of $\operatorname{Fix}(S) \cap \Xi \cap \operatorname{VI}(C, A)$ in [33, Theorem 3.1] is extended to develop the relaxed extragradient method with regularization for finding an element of $\operatorname{Fix}(S) \cap \Xi \cap \Gamma$ in our Theorem 19.

(iv) The proof of our Theorems 19 and 22 is very different from that of [33, Theorem 3.1] because our argument technique depends on Lemma 16, the restriction on the regularization parameter sequence $\left\{\alpha_{n}\right\}$, and the properties of the averaged mappings $P_{C}\left(I-\lambda_{n} \nabla f_{\alpha_{n}}\right)$ to a great extent.

(v) Because our iterative schemes (17) and (18) involve a contractive self-mapping $Q$, a $k$-strictly pseudocontractive self-mapping $S$, and several parameter sequences, they are more flexible and more subtle than the corresponding ones in [6, Theorem 5.7] and [18, Theorem 3.1], respectively.

\section{Acknowledgment}

The work of L. C. Ceng was partially supported by the National Science Foundation of China (11071169), Ph. D. Program Foundation of Ministry of Education of China (20123127110002). The work of J. C. Yao was partially supported by the Grant NSC 99-2115-M-037-002-MY3 of Taiwan. For A. Petruşsel, this work was possible with the nancial support of a grant of the Romanian National Authority for Scientific Research, CNCS-UEFISCDI, project number PNII-ID-PCE-2011-3-0094.

\section{References}

[1] Y. Censor and T. Elfving, "A multiprojection algorithm using Bregman projections in a product space," Numerical Algorithms, vol. 8, no. 2-4, pp. 221-239, 1994.

[2] C. Byrne, "Iterative oblique projection onto convex sets and the split feasibility problem," Inverse Problems, vol. 18, no. 2, pp. 441453, 2002.

[3] Y. Censor, T. Bortfeld, B. Martin, and A. Trofimov, "A unified approach for inversion problems in intensity-modulated radiation therapy," Physics in Medicine \& Biology, vol. 51, pp. 23532365, 2006.

[4] Y. Censor, T. Elfving, N. Kopf, and T. Bortfeld, “The multiplesets split feasibility problem and its applications for inverse problems," Inverse Problems, vol. 21, no. 6, pp. 2071-2084, 2005.

[5] Y. Censor, A. Motova, and A. Segal, "Perturbed projections and subgradient projections for the multiple-sets split feasibility problem," Journal of Mathematical Analysis and Applications, vol. 327, no. 2, pp. 1244-1256, 2007.

[6] H.-K. Xu, "Iterative methods for the split feasibility problem in infinite-dimensional Hilbert spaces," Inverse Problems, vol. 26, no. 10, article 105018, 2010.

[7] C. Byrne, "A unified treatment of some iterative algorithms in signal processing and image reconstruction," Inverse Problems, vol. 20, no. 1, pp. 103-120, 2004.
[8] B. Qu and N. Xiu, "A note on the CQ algorithm for the split feasibility problem," Inverse Problems, vol. 21, no. 5, pp. 1655$1665,2005$.

[9] H.-K. Xu, "A variable Krasnosel'skii-Mann algorithm and the multiple-set split feasibility problem," Inverse Problems, vol. 22, no. 6, pp. 2021-2034, 2006.

[10] Q. Yang, "The relaxed CQ algorithm solving the split feasibility problem," Inverse Problems, vol. 20, no. 4, pp. 1261-1266, 2004.

[11] J. Zhao and Q. Yang, "Several solution methods for the split feasibility problem," Inverse Problems, vol. 21, no. 5, pp. 17911799, 2005.

[12] M. I. Sezan and H. Stark, "Applications of convex projection theory to image recovery in tomography and related areas," in Image Recovery Theory and Applications, H. Stark, Ed., pp. 415462, Academic, Orlando, Fla, USA, 1987.

[13] B. Eicke, "Iteration methods for convexly constrained ill-posed problems in Hilbert space," Numerical Functional Analysis and Optimization, vol. 13, no. 5-6, pp. 413-429, 1992.

[14] L. Landweber, "An iteration formula for Fredholm integral equations of the first kind," American Journal of Mathematics, vol. 73, pp. 615-624, 1951.

[15] L. C. Potter and K. S. Arun, "A dual approach to linear inverse problems with convex constraints," SIAM Journal on Control and Optimization, vol. 31, no. 4, pp. 1080-1092, 1993.

[16] P. L. Combettes and V. R. Wajs, "Signal recovery by proximal forward-backward splitting," Multiscale Modeling \& Simulation, vol. 4, no. 4, pp. 1168-1200, 2005.

[17] G. M. Korpelevič, "An extragradient method for finding saddle points and for other problems," Ekkonomika i Matematicheskie Metody, vol. 12, no. 4, pp. 747-756, 1976.

[18] L.-C. Ceng, Q.H. Ansari, and J.-C. Yao, "An extragradient method for solving split feasibility and fixed point problems," Computers \& Mathematics with Applications, vol. 64, no. 4, pp. 633-642, 2012.

[19] N. Nadezhkina and W. Takahashi, "Weak convergence theorem by an extragradient method for nonexpansive mappings and monotone mappings," Journal of Optimization Theory and Applications, vol. 128, no. 1, pp. 191-201, 2006.

[20] J.-L. Lions and G. Stampacchia, "Variational inequalities," Communications on Pure and Applied Mathematics, vol. 20, pp. 493519, 1967.

[21] L.-C. Ceng, Q. H. Ansari, and J.-C. Yao, "Viscosity approximation methods for generalized equilibrium problems and fixed point problems," Journal of Global Optimization, vol. 43, no. 4, pp. 487-502, 2009.

[22] L.-C. Ceng and S. Huang, "Modified extragradient methods for strict pseudo-contractions and monotone mappings," Taiwanese Journal of Mathematics, vol. 13, no. 4, pp. 1197-1211, 2009.

[23] L.-C. Ceng, C.-Y. Wang, and J.-C. Yao, "Strong convergence theorems by a relaxed extragradient method for a general system of variational inequalities," Mathematical Methods of Operations Research, vol. 67, no. 3, pp. 375-390, 2008.

[24] L.-C. Ceng and J.-C. Yao, "An extragradient-like approximation method for variational inequality problems and fixed point problems," Applied Mathematics and Computation, vol. 190, no. 1, pp. 205-215, 2007.

[25] L.-C. Ceng and J.-C. Yao, "Relaxed viscosity approximation methods for fixed point problems and variational inequality problems," Nonlinear Analysis A, vol. 69, no. 10, pp. 3299-3309, 2008. 
[26] Y. Yao, Y.-C. Liou, and S. M. Kang, "Approach to common elements of variational inequality problems and fixed point problems via a relaxed extragradient method," Computers \& Mathematics with Applications, vol. 59, no. 11, pp. 3472-3480, 2010.

[27] L.-C. Zeng and J.-C. Yao, "Strong convergence theorem by an extragradient method for fixed point problems and variational inequality problems," Taiwanese Journal of Mathematics, vol. 10, no. 5, pp. 1293-1303, 2006.

[28] N. Nadezhkina and W. Takahashi, "Strong convergence theorem by a hybrid method for nonexpansive mappings and Lipschitz-continuous monotone mappings," SIAM Journal on Optimization, vol. 16, no. 4, pp. 1230-1241, 2006.

[29] W. Takahashi and M. Toyoda, "Weak convergence theorems for nonexpansive mappings and monotone mappings," Journal of Optimization Theory and Applications, vol. 118, no. 2, pp. 417428, 2003.

[30] H. Iiduka and W. Takahashi, "Strong convergence theorems for nonexpansive mappings and inverse-strongly monotone mappings," Nonlinear Analysis A, vol. 61, no. 3, pp. 341-350, 2005.

[31] A. Bnouhachem, M. Aslam Noor, and Z. Hao, "Some new extragradient iterative methods for variational inequalities," Nonlinear Analysis A, vol. 70, no. 3, pp. 1321-1329, 2009.

[32] R. U. Verma, "On a new system of nonlinear variational inequalities and associated iterative algorithms," Mathematical Sciences Research Hot-Line, vol. 3, no. 8, pp. 65-68, 1999.

[33] L.-C. Ceng, Q. H. Ansari, and J.-C. Yao, "Relaxed extragradient iterative methods for variational inequalities," Applied Mathematics and Computation, vol. 218, no. 3, pp. 1112-1123, 2011.

[34] H.-K. Xu, "Iterative algorithms for nonlinear operators," Journal of the London Mathematical Society, vol. 66, no. 1, pp. 240-256, 2002.

[35] D. P. Bertsekas and E. M. Gafni, "Projection methods for variational inequalities with application to the traffic assignment problem," Mathematical Programming Study, no. 17, pp. 139-159, 1982.

[36] D. Han and H. K. Lo, "Solving non-additive traffic assignment problems: a descent method for co-coercive variational inequalities," European Journal of Operational Research, vol. 159, no. 3, pp. 529-544, 2004.

[37] P. L. Combettes, "Solving monotone inclusions via compositions of nonexpansive averaged operators," Optimization, vol. 53, no. 5-6, pp. 475-504, 2004.

[38] G. Marino and H.-K. Xu, "Weak and strong convergence theorems for strict pseudo-contractions in Hilbert spaces," Journal of Mathematical Analysis and Applications, vol. 329, no. 1, pp. 336-346, 2007.

[39] T. Suzuki, "Strong convergence of Krasnoselskii and Mann's type sequences for one-parameter nonexpansive semigroups without Bochner integrals," Journal of Mathematical Analysis and Applications, vol. 305, no. 1, pp. 227-239, 2005.

[40] R. T. Rockafellar, "On the maximality of sums of nonlinear monotone operators," Transactions of the American Mathematical Society, vol. 149, pp. 75-88, 1970. 


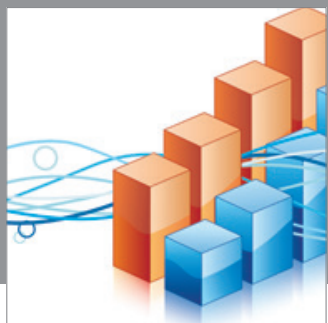

Advances in

Operations Research

mansans

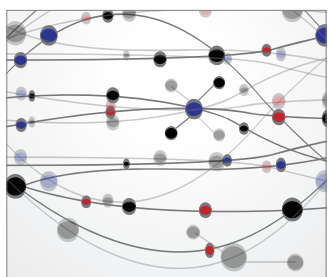

The Scientific World Journal
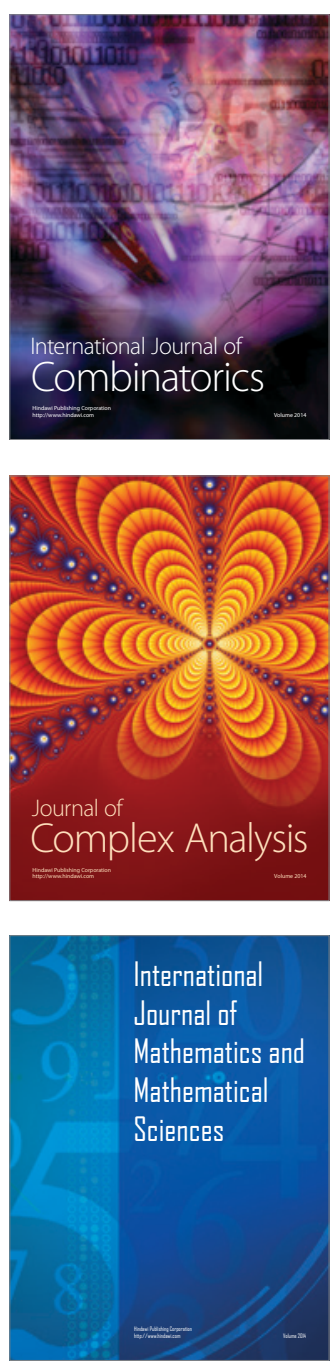
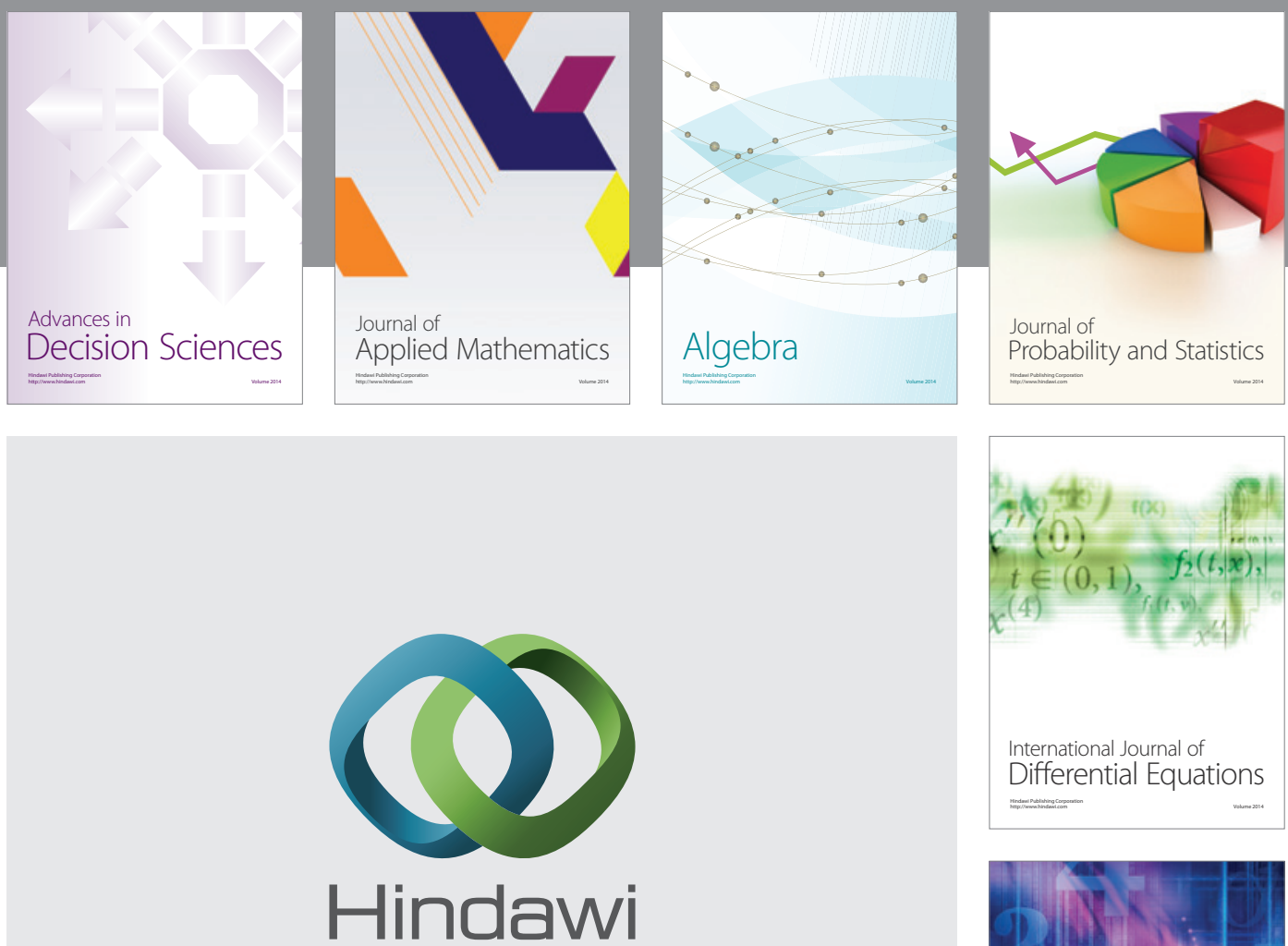

Submit your manuscripts at http://www.hindawi.com
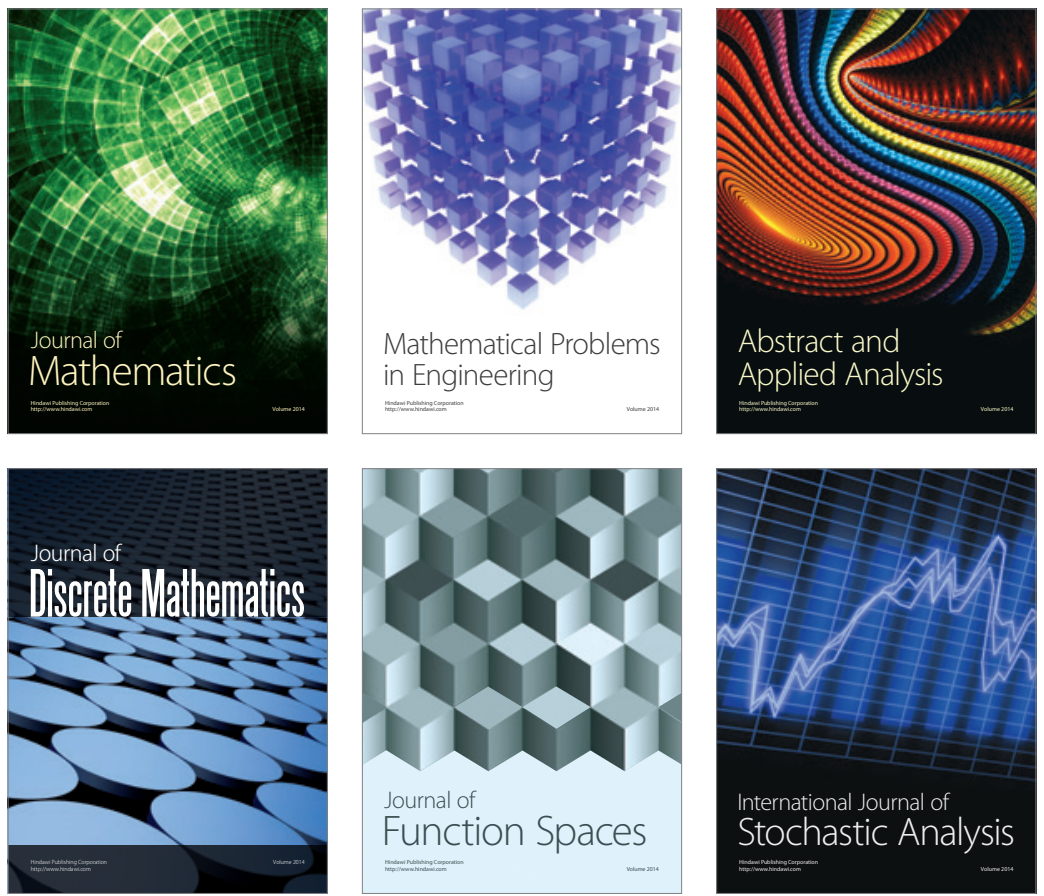

Journal of

Function Spaces

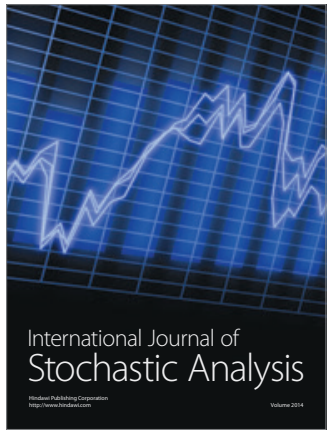

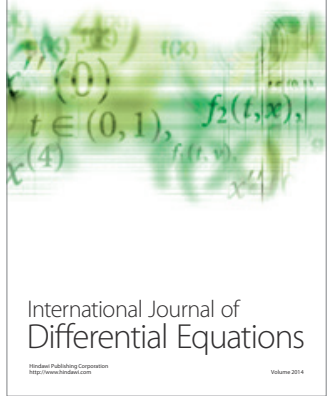
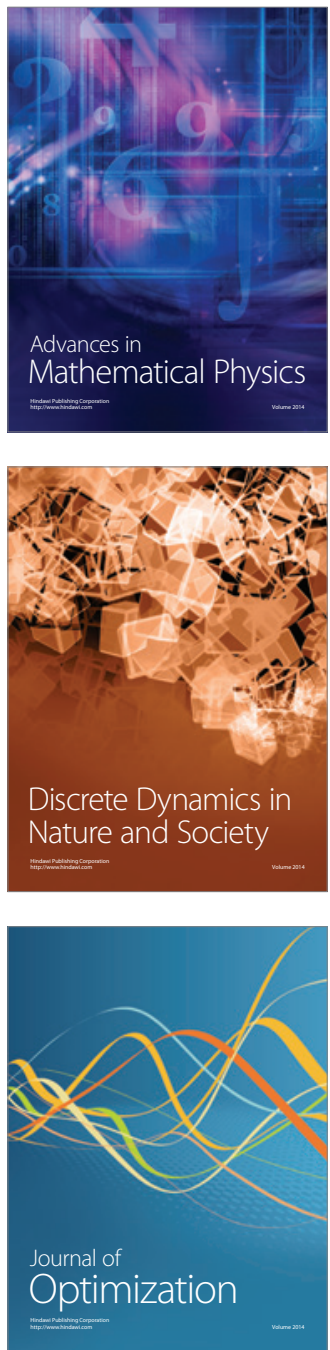